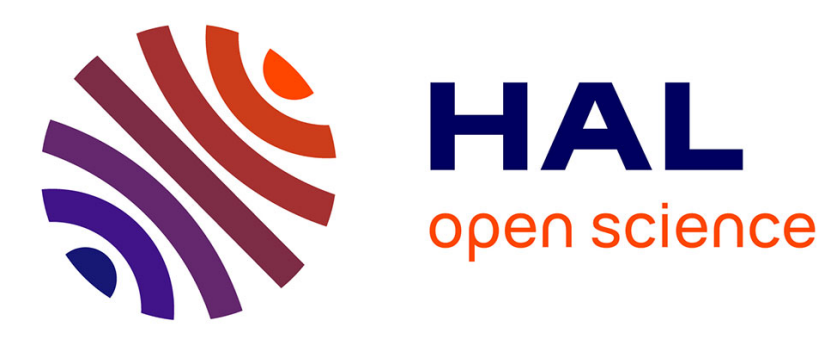

\title{
Recent Advances and Perspectives on Nonadiabatic Mixed Quantum-Classical Dynamics
}

\author{
Rachel Crespo-Otero, Mario Barbatti
}

\section{To cite this version:}

Rachel Crespo-Otero, Mario Barbatti. Recent Advances and Perspectives on Nonadiabatic Mixed Quantum-Classical Dynamics. Chemical Reviews, 2018, 118 (15), pp.7026-7068. 10.1021/acs.chemrev.7b00577 . hal-01965458

\section{HAL Id: hal-01965458 \\ https://hal-amu.archives-ouvertes.fr/hal-01965458}

Submitted on 26 Dec 2018

HAL is a multi-disciplinary open access archive for the deposit and dissemination of scientific research documents, whether they are published or not. The documents may come from teaching and research institutions in France or abroad, or from public or private research centers.
L'archive ouverte pluridisciplinaire HAL, est destinée au dépôt et à la diffusion de documents scientifiques de niveau recherche, publiés ou non, émanant des établissements d'enseignement et de recherche français ou étrangers, des laboratoires publics ou privés. 


\title{
Recent Advances and Perspectives on Nonadiabatic Mixed Quantum-Classical Dynamics
}

\author{
Rachel Crespo-Otero ${ }^{1 *}$ and Mario Barbatti ${ }^{2 *}$ \\ ${ }^{1}$ School of Biological and Chemical Sciences, Queen Mary University of London, Mile End Road, \\ London E1 4NS, United Kingdom. \\ ${ }^{2}$ Aix Marseille Univ, CNRS, ICR, Marseille, France. \\ * Corresponding authors: r.crespo-otero@qmul.ac.uk (RCO); mario.barbatti@univ-amu.fr; \\ www.barbatti.org $(M B)$
}

\section{Table of Contents}

ABSTRACT

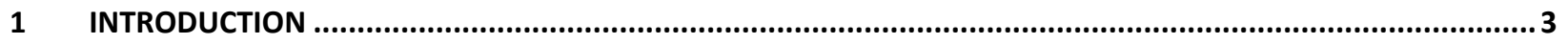

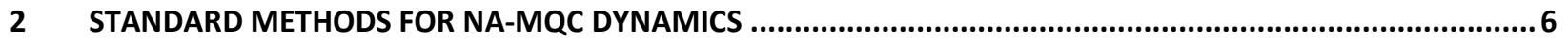

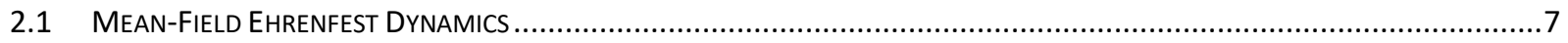

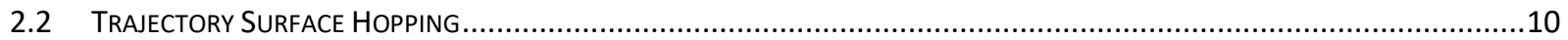

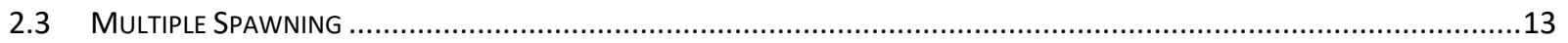

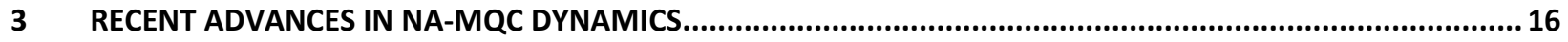

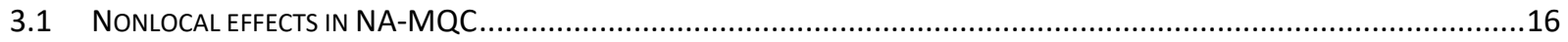

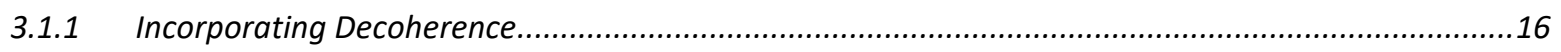

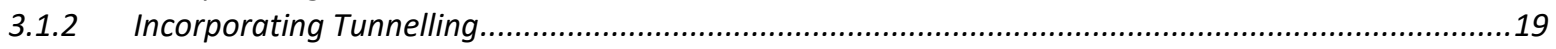

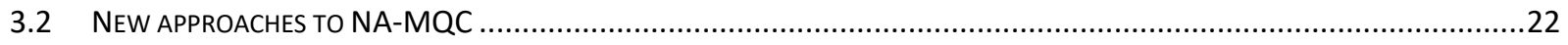

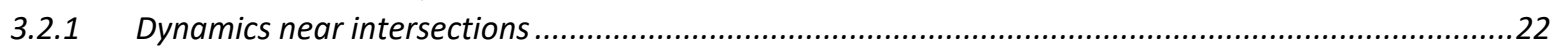

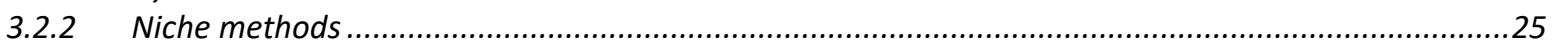

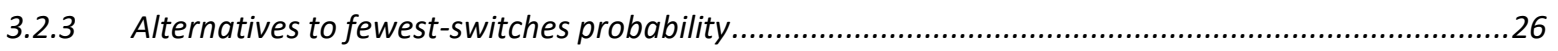

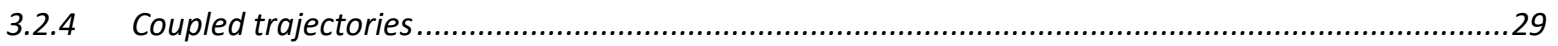

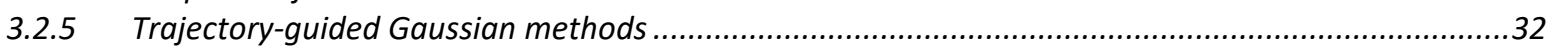

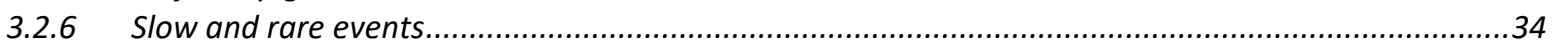

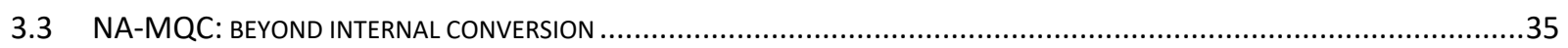

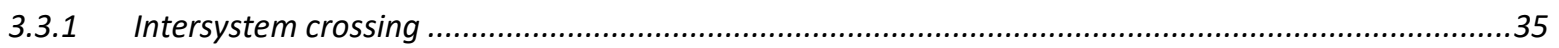

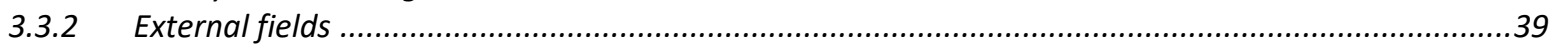

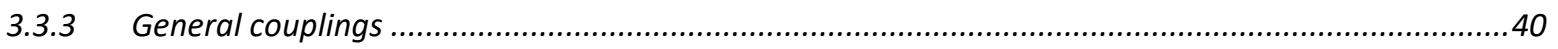

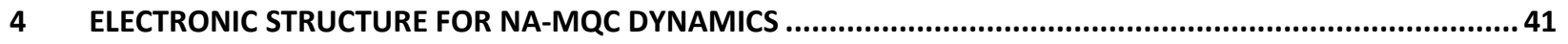

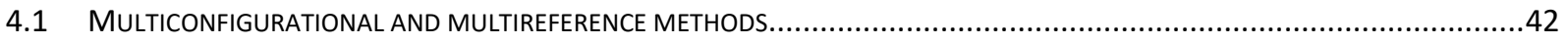

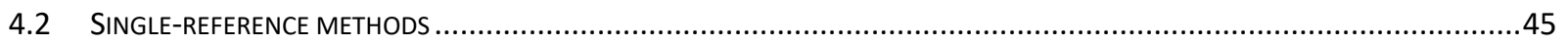

4.2.1 Nonadiabatic dynamics with single reference: does it make sense? ...........................................45

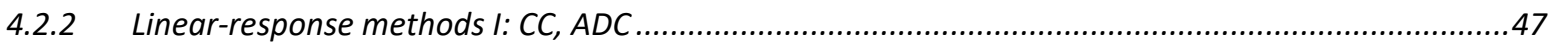

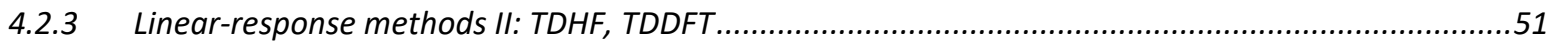


4.2.4 Real-time methods I: frozen nuclei

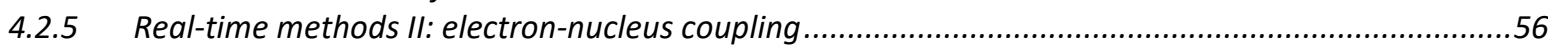

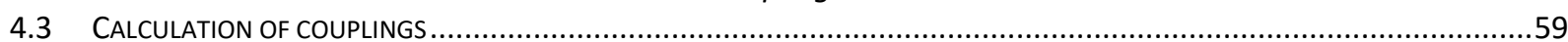

$5 \quad$ SPECTROSCOPIC SIMULATIONS BASED ON NA-MQC DYNAMICS ........................................................62

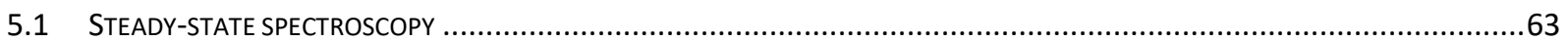

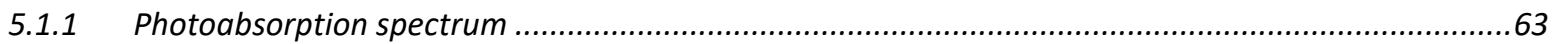

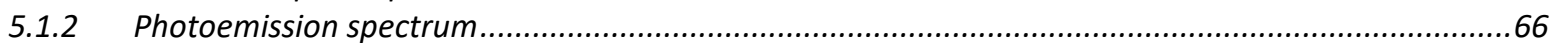

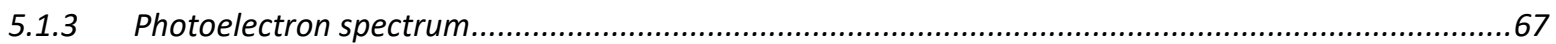

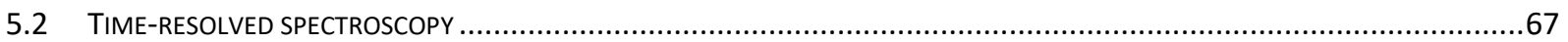

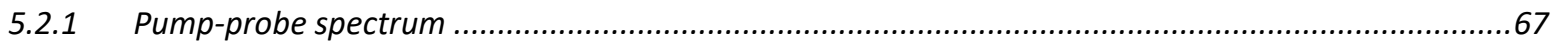

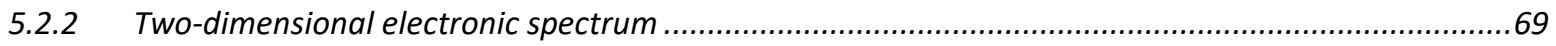

6 SOFTWARE RESOURCES FOR NA-MQC DYNAMICS .............................................................................70

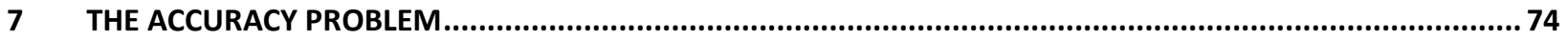

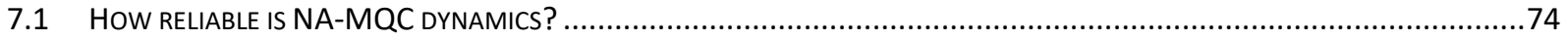

7.2 REACTION PATHS OR NA-MQC DYNAMICS: WHAT'S THE BEST QUANTUM CHEMISTRY WE CAN DO? ...............................82

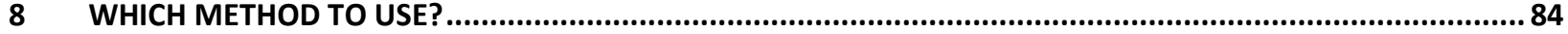

\begin{abstract}
Nonadiabatic mixed quantum-classical (NA-MQC) dynamics methods form a class of computational theoretical approaches in quantum chemistry, tailored to investigate the timeevolution of nonadiabatic phenomena in molecules and supramolecular assemblies. NA-MQC is characterized by a partition of the molecular system into two subsystems, one to be treated quantum-mechanically (usually, but not restricted to electrons); and another to be dealt with classically (nuclei). The two subsystems are connected through nonadiabatic couplings terms, to enforce self-consistency. A local approximation underlies the classical subsystem, implying that direct dynamics can be simulated, without needing pre-computed potential energy surfaces. The NA-MQC split allows reducing computational costs, enabling the treatment of realistic molecular systems in diverse fields. Starting from the three most well-established methods-mean-field Ehrenfest, trajectory surface hopping, and multiple spawning, this review focus on the NA-MQC dynamics methods and programs developed in the last ten years. It stresses the relations between approaches and their domains of application. The electronic structure methods most commonly used together with NA-MQC dynamics are reviewed as well. The accuracy and precision of NAMQC simulations are critically discussed, and general guidelines to choose an adequate method for each application are delivered.
\end{abstract}




\section{Introduction}

Photochemical and photophysical phenomena in molecules, supramolecular assemblies, and solids involve the time evolution of the electronic population through a manifold of electronic states. Modeling these processes requires considering the coupling between the nuclear and electronic motions beyond the adiabatic regime. The high computational costs of such simulations have led to the development of different strategies. On the one hand, it is possible to tackle the problem fully quantum mechanically but at reduced dimensionality by exclusively treating, for instance, the electron dynamics in a frozen nuclear frame or incorporating few nuclear modes. On the other hand, full dimensionality may be retained at the cost of splitting the system between a set of degree of freedom to be treated fully quantum mechanically and another set to be treated classically. This second strategy is the basis of the Nonadiabatic Mixed Quantum-Classical (NAMQC) dynamics explored in this review.

NA-MQC dynamics is a general umbrella under which we may classify several different approaches developed to deal with time-resolved simulations over the last forty years. Among these approaches, we may include trajectory surface hopping (TSH), mean-field Ehrenfest (MFE), mixed quantum-classical Liouville equation (QCLE), ${ }^{1-3}$ the mapping approach, ${ }^{4-5}$ multiple spawning (MS), ${ }^{6-7}$ nonadiabatic Bohmian dynamics (NABDY), ${ }^{8-9}$ and the recently proposed coupled-trajectories mixed quantum-classical (CT-MQC) method. ${ }^{10}$ Naturally, as in any classification, there is a degree of arbitrariness: should MS be still considered an NA-MQC approach, as it ultimately recovers the information on the nuclear wave packet? We broadly define the NA-MQC methods as those propagating the nuclei (or more generally, slow particles) via classical trajectories. We believe, however, that it is not productive to focus on such a taxonomic question. In the interest of pragmatism, we instead assume some porous boundaries and discuss methods that incorporate full dimensional treatment of electrons and nuclei, the inclusion of nonadiabatic transitions, and some type of classical/quantum partition. Fig. 1 schematically illustrates the hierarchic relation between some of the key methods for nonadiabatic dynamics.

With this definition in mind, we prepared this review focusing on methods, rather than on applications. Nonetheless, it would be yet a Homeric work to attempt to survey all classes of NAMQC methods. For this reason, we have narrowed our focus even further to NA-MQC methods often used in conjunction with direct (or on-the-fly) ${ }^{11}$ calculations of electronic structure properties 
(in opposition to methods that have been mostly applied with model Hamiltonians). In the last fifteen years or so, on-the-fly NA-MQC dynamics has been pushing the boundaries of excitedstate computation chemistry, becoming a central tool for investigating practical problems in diverse fields. ${ }^{6,12-15}$

In the sub-class of on-the-fly NA-MQC methods, we first examine the three cornerstone approaches - mean field Ehrenfest, trajectory surface hopping, and multiple spawning (Section 2.3). From them, we guide the reader through a myriad of new methods that have been developed, especially in the last decade (Section 3). The equations of motion (EOM) for the main methods are written out, sharing a standard notation to emphasize the relations between them. The used symbols are outlined in Table 1.

Due to the narrow focus, with few exceptions, we will not discuss methods related to NABDY, QCLE, and the mapping approach. The first class is reviewed in Ref. ${ }^{16}$. An excellent introduction to the latter two classes of methods can be found in Ref. ${ }^{17}$. Concerning QCLE, we also recommend Ref. ${ }^{18}$ for details on the momentum-jump (MJ) QCLE and the generalized quantum master equation (GQME) approaches.

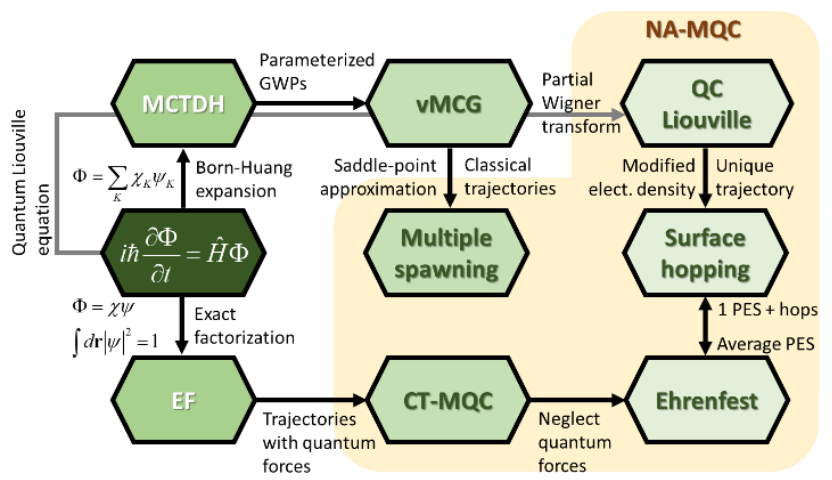

Fig. 1 Schematic relation between methods for nonadiabatic dynamics. Starting from the exact non-relativistic timedependent Schrödinger equation (center, left), the full molecular problem may be solved either via a Born-Huang expansion (as done in MCTDH), an exact factorization (EF) of the molecular wavefunction, or propagation of the density via Liouville equations. In the Born-Huang branch, MCTDH combined with Heller's frozen Gaussian wave packets (GWP) approach renders the vMCG approximation, which, in the limit of a coherent GWPs, converges to Multiple Spawning (MS).$^{19}$ In the EF branch, a trajectory approximation of the nuclear wave packet leads to the CTMQC method, in which trajectories are coupled by quantum forces. If these quantum forces are neglected, the method reduces to the mean field Ehrenfest (MFE) approach. ${ }^{10}$ The connection between MFE and vMCG is discussed in Ref. ${ }^{20}$. If instead of propagating the trajectories on an average potential energy surface, they are propagated on a single surface, which can be stochastically exchanged by another, surface hopping (TSH) is recovered. In the third branch, the quantum density is propagated via Liouville equations. The mixed-quantum-classical limit of the partial Wigner trasform of the density gives rise to the quantum-classical Liouville equations (QCLE). From QCLE, assuming unique trajectories, large nuclear velocities, and modifying the electronic density matrix leads to fewest switches TSH. ${ }^{21}$ If one assumes some soft boundaries in the classification, the NA-MQC methods may be identified with the methods 
propagating the nuclei through classical trajectories, which includes multiple spawning, CT-MQC, Ehrenfest, QCLE, and surface hopping. This chart presents some of the main approaches in the field, but it is far from representing the broad variety of alternatives available. ${ }^{17,22}$

The importance that on-the-fly NA-MQC dynamics has acquired in computational chemistry rests on how general it became thanks to interfaces between dynamics algorithms and general electronic structure methods. We cover this relation as well, discussing the leading electronic structure methods that have been employed for NA-MQC simulations (Section 4). At this point, we prefer to assume a critical perspective, focusing our account on the limitations and potential problems of each of these methods.

Table 1. Table of symbols recurrently used in the text.

\begin{tabular}{|c|c|}
\hline Symbol & Definition \\
\hline$\Phi$ & Molecular wavefunction \\
\hline$\Psi, \psi_{K}$ & Electronic wavefunction \\
\hline$\chi$ & Nuclear wavefunction \\
\hline$\Theta, \varphi, \vartheta$ & Slater determinant, molecular orbital, atomic orbital \\
\hline$c_{K}, A_{K}$ & Electronic and nuclear time-dependent coefficients \\
\hline$\rho$ & Density matrix \\
\hline$S_{J K}$ & Wavefunction overlap \\
\hline$\hat{H}, \hat{H}_{e}$ & Molecular and electronic Hamiltonian \\
\hline$\hat{K}_{n}, \hat{K}_{e}$ & Nuclear and electronic kinetic energies \\
\hline$\hat{T}, \hat{\tau}$ & Cluster operator, excitation operator \\
\hline $\begin{array}{l}E_{e}, E_{K} \\
v\end{array}$ & $\begin{array}{c}\text { Electronic energy, adiabatic energy } \\
\text { Potential }\end{array}$ \\
\hline $\mathbf{F}, \mathbf{G}$ & Force, energy gradient \\
\hline$\sigma_{J K}^{N A C}$ & Time-derivative nonadiabatic coupling \\
\hline$\sigma_{J K}^{S O C}, \sigma_{J K}^{E M C}$ & Spin-orbit coupling, radiation-matter coupling \\
\hline $\mathbf{d}_{J K}$ & Nonadiabatic coupling vector \\
\hline$\mu_{J K}, f_{J K}$ & Transition dipole moment, oscillator strength \\
\hline $\begin{array}{c}I, J, K, L \\
\alpha\end{array}$ & $\begin{array}{l}\text { Index for electronic states; } L \text { is the active state } \\
\text { Index of nuclei }\end{array}$ \\
\hline$N$ & Index for trajectories of ensemble points \\
\hline$i, j ; a, b$ & Indexes for occupied and unoccupied orbitals \\
\hline $\mathbf{R}, \mathbf{r}$ & Nuclear and electronic coordinates \\
\hline $\mathbf{v}, \mathbf{P}, \mathbf{M}$ & Nuclear velocity, momentum, and mass \\
\hline$\overline{\mathbf{A}}, \hat{\mathbf{A}}$ & Classical value and operator of observable $\mathbf{A}$ \\
\hline$t, \tau$ & Time, decoherence time \\
\hline$P, \eta$ & Probability, distribution \\
\hline
\end{tabular}


The development of time-resolved spectroscopy has revolutionized the way we explore chemical systems. ${ }^{23-26}$ The information delivered by these experimental methods, however, needs to be deconvoluted, which has raised the importance of computational chemistry. Theoretical simulations are now vital ingredients for the analysis of any advanced experimental set of data; and NA-MQC dynamics plays an important role for that, naturally providing time-resolved information. In Section 5, we discuss how NA-MQC dynamics has been used to simulate several spectroscopic techniques directly.

With the popularization of the NA-MQC methods, several computational programs dedicated to NA-MQC dynamics (or having NA-MQC dynamics incorporated as an auxiliary algorithm) have been developed and released in the last ten years or so. We are ourselves developers of one of such programs, the Newton-X platform. In Section 6, we survey these implementations, but we already anticipate this part of the review will be quickly outdated, given the frenetic rate of new developments released nowadays.

NA-MQC dynamics comes at a cost. Hundreds of thousands of CPU hours may be required to simulate a single molecule. Researchers have coped with such cost by both developing new optimized techniques and downgrading theoretical levels. The price to pay for this second strategy may be too high, leading to unacceptable loss of accuracy. This problem is discussed in Section 7.

As specialists in the field, developing a major program platform for NA-MQC dynamics and applying these methods to investigate many different systems, we have accumulated an experience that we believe may be useful to share. Throughout the review, especially in Section 8 , we lay down a series of recommendations on methods and procedures. We hope they will be useful not only for beginners in the field but also for experienced researchers, who may re-evaluate their own choices. Naturally, these are educated but somewhat subjective opinions. The reader will always be warned when this is the case.

\section{Standard methods for NA-MQC dynamics}

Three of the most traditional NA-MQC dynamics methods for treating nonadiabatic phenomena are the MFE, SH, and MS. Each of them tackles the nonadiabatic process in an entirely different way either by averaging electronic states (MFE), hopping between states (TSH), or spawning new basis functions to other states (MS). These methods have been discussed and 
reviewed in detail in Refs. ${ }^{16,27-32}$. In this section, we only outline their main features, which will be useful to discuss the new developments that have been recently proposed.

In common, these three types of methods share a treatment of nuclear motion in terms of classical trajectories (which in the case of MS are used as an auxiliary grid for a quantum propagation of the nuclei). As a consequence, at each time step of a trajectory evolution, they require computation of electronic quantities (potential energies, energy gradients, couplings, etc.) for the classical position of the nuclei (local approximation). Such approximation has a significant impact on computational costs because pre-computed multidimensional surfaces for electronic coordinates are not required anymore. Instead, these methods may be implemented as to compute these quantities on-the-fly during the trajectory integration. Naturally, the classical localization of nuclei is also the drawback of these methods, as they fail to provide a description of quantum phenomena depending on global features (like tunneling, for instance).

\subsection{Mean-Field Ehrenfest Dynamics}

We start with the time-dependent Schrödinger equation (TDSE)

$$
i \hbar \frac{\partial \Phi}{\partial t}=\hat{H} \Phi
$$

where $\Phi$ is the total (non-relativistic) molecular wavefunction. The full Hamiltonian in this equation is taken as

$$
\hat{H}=\hat{K}_{n}+\hat{H}_{e}
$$

where $\hat{K}_{n}$ is the kinetic energy operator for the slow particles (usually nuclei) and $\hat{H}_{e}$ is the Hamiltonian for the fast particles (usually, but not necessarily only electrons ${ }^{33-34}$ ).

In the mean-field approximation, the molecular wavefunction is factorized in terms of a function of coordinates $\mathbf{r}$ describing the fast particles and a function of coordinates $\mathbf{R}$ describing the slow particles: ${ }^{35}$

$$
\Phi(\mathbf{r}, \mathbf{R}, t)=\chi(\mathbf{R}, t) \Psi(\mathbf{r}, t) \exp \left(\frac{i}{\hbar} \int_{t_{0}}^{t} d t^{\prime} E_{e}\left(t^{\prime}\right)\right)
$$


where the phase factor is $E_{e}=\left\langle\Phi\left|\hat{H}_{e}\right| \Phi\right\rangle$.

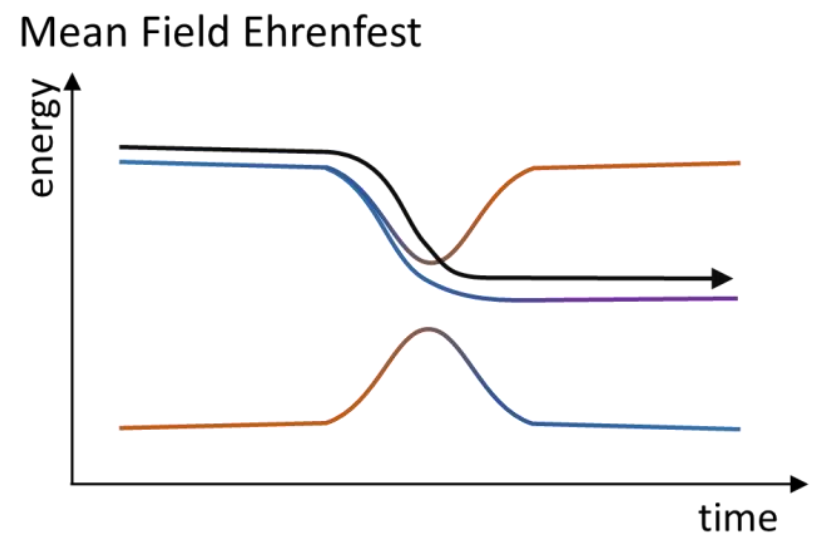

Fig. 2 Schematic illustration of the mean field Ehrenfest (MFE) dynamics. A trajectory is run on a surface averaged over all electronic states weighted by their respective electronic population.

After replacing this wavefunction Ansatz, Eq. (3), in Eq. (1), the TDSE can be projected in the fast-coordinates space and in the slow-coordinate space leading to two coupled timedependent equations for $\chi$ and $\Psi$. The classical limit $(\hbar \rightarrow 0)$ of the equation for $\chi$ can be easily shown ${ }^{35}$ to be equivalent to Newton's equations for the motion of each slow particle $\alpha$ (with classical coordinate $\overline{\mathbf{R}}$ and mass $M_{\alpha}$ ) on the average potential of the fast particles:

$$
\frac{d^{2} \overline{\mathbf{R}}}{d t^{2}}=\frac{1}{M_{\alpha}} \mathbf{F}(\overline{\mathbf{R}})
$$

where

$$
\mathbf{F}(\overline{\mathbf{R}}) \equiv-\nabla_{\alpha}\left\langle\Psi(\mathbf{r}, t)\left|\hat{H}_{e}(\mathbf{r} ; \overline{\mathbf{R}})\right| \Psi(\mathbf{r}, t)\right\rangle_{\mathbf{r}}
$$

The fast particles, in turn, evolve according to

$$
i \hbar \frac{\partial \Psi(\mathbf{r}, t ; \overline{\mathbf{R}})}{\partial t}=\hat{H}_{e}(\mathbf{r} ; \overline{\mathbf{R}}) \Psi(\mathbf{r}, t ; \overline{\mathbf{R}}),
$$

where we have made explicit the parametric dependence of the electronic wavefunction on the classical nuclear coordinate. (For the complete derivation of Eqs. (4) and (6), see Ref. ${ }^{16}$.) 
The classical equation of motion (EOM), Eq. (4), can be integrated with standard methods, as the velocity Verlet algorithm. ${ }^{36}$ The quantum EOM, Eq. (6), can be solved numerically along the classical trajectories without a need of choosing basis functions. Alternatively, if the fast particles correspond to electrons, the time-dependent electronic wavefunction $\Psi$ can be expanded as a linear combination of electronic states:

$$
\Psi(\mathbf{r}, t ; \overline{\mathbf{R}})=\sum_{K} c_{K}(t) \psi_{K}(\mathbf{r} ; \overline{\mathbf{R}})
$$

where $\psi_{K}$ are electronic wavefunctions for state $K$, with parametrical dependence on the classical nuclear coordinates $\overline{\mathbf{R}}(t)$. If this multiconfigurational approach is used, the quantum EOM (Eq. (6)) is reduced to 35

$$
\frac{d c_{J}}{d t}=\sum_{K}-c_{K}\left(\frac{i}{\hbar} H_{J K}+\sigma_{J K}^{N A C}\right) .
$$

In this equation,

$$
H_{J K}(\overline{\mathbf{R}}) \equiv\left\langle\psi_{J}\left|\hat{H}_{e}\right| \psi_{K}\right\rangle
$$

and

$$
\sigma_{J K}^{N A C}(\overline{\mathbf{R}}) \equiv\left\langle\psi_{J} \mid \frac{\partial \psi_{K}}{\partial t}\right\rangle=\mathbf{d}_{J K} \cdot \overline{\mathbf{v}}
$$

In the last equation,

$$
\mathbf{d}_{J K} \equiv\left\langle\psi_{J} \mid \nabla \psi_{K}\right\rangle
$$

is the nonadiabatic coupling (NAC) vector and $\overline{\mathbf{v}}$ is the classical nuclear velocity. The coefficients $c_{J}$ define a density matrix $\rho$ whose diagonal terms $\rho_{J J} \equiv c_{J} c_{J}^{*}$ are the populations, and the offdiagonal terms $\rho_{I J} \equiv c_{I} c_{J}^{*}$ are the coherences.

Still with the expansion in Eq. (7), the force acting on the nuclei is

$$
\mathbf{F}(\overline{\mathbf{R}})=-\sum_{I J} c_{I}^{*} c_{J}\left\langle\psi_{I}\left|\nabla \hat{H}_{e}\right| \psi_{J}\right\rangle
$$


Particular expressions for the force in the adiabatic and diabatic representations are given in Eqs. (29) and (30) of Ref. ${ }^{37}$. The implementation of a second-order Ehrenfest method based on CASSCF, in which Hessian information is used to increase integration time steps in the classical EOM, is discussed in Ref. ${ }^{38}$.

To summarize, in MFE, the system is propagated by simultaneously solving the quantum EOM for the classical coordinates $\overline{\mathbf{R}}$, Eq. (8), to obtain the matrix elements of $\mathbf{c}$; and the classical EOM, Eq. (4) with the average force in Eq. (12), to obtain $\overline{\mathbf{R}}$. The nuclear motion on the averaged potential energy surface is schematically illustrated in Fig. 2.

Because of the average description of the potential, MFE dynamics cannot represent different physical situations found when a system leaves regions of strong NACs. Moreover, MFE does not satisfy the principle of detailed balance, ${ }^{35,39}$ which means that at equilibrium a forward process is not balanced by its reverse process. The inclusion of quantum corrections through a modified symmetric coupling matrix element may produce Boltzmann distributions in the longtime limit. ${ }^{40-41}$ This approach, however, is restricted to propagation in the diabatic representation. The MFE approach, with emphasis on its more recent multiconfigurational variants, has been recently reviewed in Ref. $^{22}$ (see also Section 3.2.5). We further discuss the MFE approach in the context of real-time single-reference methods in Sections 4.2.4 and 4.2.5.

\subsection{Trajectory Surface Hopping}

In trajectory surface hopping (TSH), sometimes also called molecular dynamics with quantum transitions (MDQT), ${ }^{42}$ a swarm of classical and independent trajectories approximates the evolution the nuclear wave packet evolving on individual Born-Oppenheimer (BO) surfaces. Nonadiabatic transitions are considered using a stochastic algorithm to decide whether the system will stay on the current electronic state or hop to another one (Fig. 3). ${ }^{43}$ Because of its conceptual simplicity and straightforward implementation, TSH is likely the most popular NA-MQC method. 


\section{Surface Hopping}

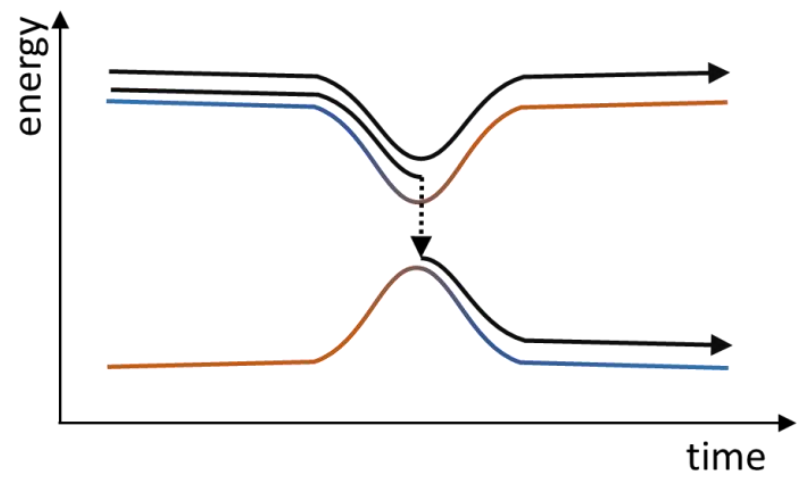

Fig. 3 Schematics illustration of trajectory surface hopping (TSH). An ensemble of independent trajectories is propagated on single BO surfaces. Random events allow trajectories to change the surface mainly at coupling regions.

Although TSH has been in use since the early 1970s, it was only in 1990 that it gained its most famous formulation, the fewest-switches surface hopping algorithm (FSSH). ${ }^{43}$ In this approach, the electronic time-evolution is obtained via the quantum EOM given in Eq. (8) (the same one used in MFE), while the nuclear dynamics for each nucleus $\alpha$ is propagated on a single BO potential energy surface (PES) of a state $L$

$$
\frac{d^{2} \overline{\mathbf{R}}_{\alpha}}{d t^{2}}=-\frac{1}{M_{\alpha}} \nabla_{\alpha} H_{L L} .
$$

(In an adiabatic basis, $H_{L L}$ is simply the adiabatic energy $E_{L}$.)

During the propagation, the instantaneous probability that the trajectory will nonadiabatically hop from state $L$ to a state $J$ is given by

$$
\begin{aligned}
P_{L \rightarrow J}^{F S S H} & =\max \left[0, \frac{2 \Delta t}{\left|c_{L}\right|^{2}}\left(\hbar^{-1} \operatorname{Im}\left(H_{L J} c_{J} c_{L}^{*}\right)-\operatorname{Re}\left(\sigma_{L J}^{N A C} c_{J} c_{L}^{*}\right)\right)\right] \\
& =\max \left[0, \frac{2 \Delta t}{\rho_{L L}}\left(\hbar^{-1} \operatorname{Im}\left(H_{L J} \rho_{J L}\right)-\operatorname{Re}\left(\sigma_{L J}^{N A C} \rho_{J L}\right)\right)\right]
\end{aligned}
$$

which in the adiabatic basis simplifies to 


$$
\begin{aligned}
P_{L \rightarrow J}^{F S S H} & =\max \left[0, \frac{-2 \Delta t}{\left|c_{L}\right|^{2}} \operatorname{Re}\left(\sigma_{L J}^{N A C} c_{J} c_{L}^{*}\right)\right] \\
& =\max \left[0, \frac{-2 \Delta t}{\rho_{L L}} \operatorname{Re}\left(\sigma_{L J}^{N A C} \rho_{J L}\right)\right]
\end{aligned}
$$

Whether a hopping event from $L$ to $J$ happens or not is estimated by sampling a random number $r_{t}$ $([0,1])$ and evaluating the following condition:

$$
\sum_{K=1}^{J-1} P_{L \rightarrow K}^{F S S H}<r_{t} \leq \sum_{K=1}^{J} P_{L \rightarrow J}^{F S S H}
$$

In addition to the inequality (16), some criterion for the conservation of energy is also generally imposed, usually by rescaling the velocity after the hopping in the direction of the NAC vector by a value corresponding to the potential energy gap at the hopping time. ${ }^{44}$ The rescaling in the NAC directions is motivated by the Pechukas force occurring during the nonadiabatic transition. ${ }^{2,45-46}$ If NAC vectors are not available, the velocity is sometimes rescaled in the direction of the momentum, which is an ad hoc procedure to grant energy conservation without further justification. If no scaling can enforce energy conservation, the hop event is not allowed (forbidden or frustrated hop) ${ }^{35}$ For a discussion on how to treat the momentum in case of forbidden hops, see Ref. ${ }^{17}$, P. 279-280, and references therein. In the method variant named fewest switches with time uncertainty (FSTU), the Heisenberg uncertainty principle is invoked to allow the classically forbidden hop to occur at a nearby geometry. ${ }^{47}$

In practical terms, the integration of the quantum and classical EOMs (Eq. (8) and Eq. (13) ) is not done with the same time steps. While the classical EOM requires time steps of about 0.1 to $0.5 \mathrm{fs}$, the fast oscillations in the quantum EOM require much shorter steps, 0.005 to 0.01 fs. If energies, forces, and nonadiabatic coupling were to be computed at shorter steps as every 0.005 fs, NA-MQC dynamics would not be possible due to the computational costs. Thus, commonly, these electronic quantities are calculated only at the classical steps. The values used for integration of the quantum steps are given by interpolation between subsequent classical steps.

Despite its success, FSSH is an ad hoc theory, not directly derived from first principles. Subotnik and coworkers ${ }^{21}$ and, later, Kapral ${ }^{48}$ have recently discussed how FSSH can be connected to QCLE; a first-principle approach developed since the nineties by Martens ${ }^{1,49}$ and Kapral, ${ }^{2-3}$ and 
more recently by Markland. ${ }^{18}$ In Ref. ${ }^{21}$, it is shown that FSSH can be approximately derived from QCLE provided that two major conditions are satisfied: first, the nuclei should be moving quickly; and secondly, there are no explicit interference effects between nuclear wave packets. In addition, decoherence corrections based on forces differences must be considered as well, an element missed in the FSSH formulation discussed above (see Section 3.1.1). The connection of FSSH to QCLE has also been applied to derive formal ways to evaluate diabatic populations, and expectation values for a TSH propagated in adiabatic representation, as well as to generate initial conditions for an electronic state that is not an adiabatic wavefunction at time zero. ${ }^{50}$

Different from MFE, FSSH in the adiabatic representation approximately satisfies the principle of detailed balance. ${ }^{51-52}$

\subsection{Multiple Spawning}

The multiple spawning (MS) method ${ }^{6-7}$ expands the nuclear wavefunction by Gaussian functions that are propagated as classical trajectories. In its exact formal framework, MS is also known as full multiple spawning (FMS). When MS is connected to a particular electronic structure method, it is commonly called ab initio multiple spawning (AIMS).

In MS, the number of nuclear functions $\left(N_{K}(t)\right)$ is allowed to change through spawning events, to represent the bifurcation of the wave packet in regions of significant nonadiabatic couplings (Fig. 4) ${ }^{53-54}$ Historically, the first on-the-fly NA-MQC simulation based on an ab initio method was done with MS employing generalized valence bond (GVB) wavefunctions. ${ }^{55}$ (One year before, an on-the-fly TSH had been reported but based on a semiempirical method. ${ }^{56}$ )

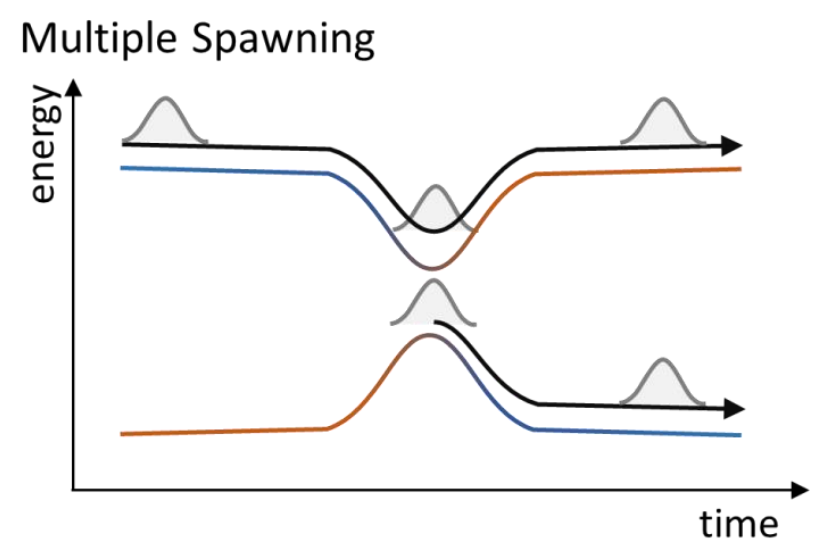


Fig. 4 Schematic illustration of multiple spawning (MS). A classical trajectory serves as the center for a generalized Gaussian wave packet. In the coupling region, new Gaussians may be created to explore other surfaces.

The derivation of MS starts from a Born-Huang expansion of the total wavefunction

$$
\Phi(\mathbf{r}, \mathbf{R}, t)=\sum_{K} \chi_{K}(\mathbf{R}, t) \psi_{K}(\mathbf{r} ; \mathbf{R})
$$

The nuclear wave packet $\chi_{K}$ is written as a linear combination of multidimensional frozen Gaussian functions $g_{K}^{m}$ with time-dependent coefficients $A_{K}^{m}$ and $N_{f}$ degrees of freedom:

$$
\chi_{K}(\mathbf{R}, t)=\sum_{m=1}^{N_{K}(t)} A_{K}^{m}(t) g_{K}^{m}\left(\mathbf{R} ; \overline{\mathbf{R}}_{K}^{m}(t), \overline{\mathbf{P}}_{K}^{m}(t), \gamma_{K}^{m}(t), \alpha_{K}^{m}\right)
$$

where

$$
g_{K}^{m}\left(\mathbf{R} ; \overline{\mathbf{R}}_{K}^{m}, \overline{\mathbf{P}}_{K}^{m}, \gamma_{K}^{m}\right)=\left(\frac{2 \alpha}{\pi}\right)^{N_{f} / 4} \exp \left(-\alpha\left(\mathbf{R}-\overline{\mathbf{R}}_{K}^{m}\right)^{2}+i \hbar^{-1} \overline{\mathbf{P}}\left(\mathbf{R}-\overline{\mathbf{R}}_{K}^{m}\right)+i \hbar^{-1} \gamma_{K}^{m}\right)
$$

The Gaussian widths $(\alpha)$ are time-independent parameters, ${ }^{57}$ the nuclear phase $\left(\gamma_{K}^{m}\right)$ is propagated semiclassically ${ }^{53,58}$

$$
\frac{\partial \gamma_{K}^{m}}{\partial t}=-H_{K K}\left(\mathbf{R}_{K}^{m}\right)+\sum_{i=1}^{3 N} \frac{\left(P_{K i}^{m}\right)^{2}}{2 M_{i}}
$$

and the position-momentum Gaussian centers $\left(\overline{\mathbf{R}}_{K}^{m}\right.$ and $\left.\overline{\mathbf{P}}_{K}^{m}\right)$ are propagated classically

$$
\begin{aligned}
\frac{d \overline{\mathbf{R}}_{K}^{m}}{d t} & =\frac{\overline{\mathbf{P}}_{K}^{m}}{M_{\alpha}} \\
\frac{d \overline{\mathbf{P}}_{K}^{m}}{d t} & =-\nabla_{\alpha} H_{K K}\left(\overline{\mathbf{R}}_{K}^{m}\right) .
\end{aligned}
$$

Eqs. (17) and (18) are inserted into the TDSE (Eq. (1)), which is then projected on a particular state $(J, m)$, resulting in an $\mathrm{EOM}$ for $\mathbf{A}_{J}$ :

$$
\frac{d \mathbf{A}_{J}}{d t}=-i\left(\mathbf{S}_{J J}\right)^{-1}\left\{\left[\mathbf{H}_{J J}-i \dot{\mathbf{S}}_{J J}\right] \mathbf{A}_{J}+\sum_{K \neq J} \mathbf{H}_{J K} \mathbf{A}_{K}\right\}
$$


where the matrix elements are given by $\mathbf{S}_{I l, J m}=\left\langle g_{I}^{l} \mid g_{J}^{m}\right\rangle_{\mathbf{R}} \delta_{I J}, \dot{\mathbf{S}}_{I l, J m}=\left\langle g_{I}^{l} \mid \frac{\partial}{\partial t} g_{J}^{m}\right\rangle_{\mathbf{R}} \delta_{I J}$, and $H_{I l, J m}=\left\langle g_{I}^{l} \psi_{I}\left|\hat{K}_{n}+\hat{H}_{e}\right| g_{J}^{m} \psi_{J}\right\rangle_{\mathbf{R}, \mathbf{r}}$. The evaluation of the Hamiltonian matrix elements is the bottleneck of MS simulations. A zero-order saddle point approximation (SPA) is assumed to calculate these integrals: ${ }^{53}$

$$
\begin{aligned}
\left\langle g_{I}^{l} \Psi_{I}|\hat{\mathbf{O}}| g_{J}^{m} \Psi_{J}\right\rangle_{\mathbf{R}, \mathbf{r}} & =\left\langle g_{I}^{l}\left|\mathbf{O}_{I J}(\mathbf{R})\right| g_{J}^{m}\right\rangle_{\mathbf{R}} \\
& \approx\left\langle g_{I}^{l} \mid g_{J}^{m}\right\rangle_{\mathbf{R}} \mathbf{O}_{I J}(\tilde{\mathbf{R}}),
\end{aligned}
$$

where $\tilde{\mathbf{R}}$ is the centroid of the product of the functions $g_{I}^{l}$ and $g_{J}^{m}$. This approximation allows calculating the required parameters on-the-fly. The SPA is applied to compute adiabatic energies $E_{I}(\tilde{\mathbf{R}})$ and nonadiabatic couplings $\mathbf{d}_{J K}(\tilde{\mathbf{R}})$. Because both should be determined at the centroid $\tilde{\mathbf{R}}$, it implies that additional electronic structure calculations should be done at each time step. A bra-ket approximation (BAT), which only uses quantities computed at $\overline{\mathbf{R}}$, has been proposed in the zeroth ${ }^{59}$ and first order ${ }^{60}$ to reduce these costs.

The most prominent feature of the MS approach is the spawning of new basis functions to represent the wavefunction bifurcation after leaving the region of significant nonadiabatic coupling. The spawning algorithm is explained in details in Ref. ${ }^{53}$. At each time step, nonadiabatic couplings $\mathbf{d}_{J K}$ (appearing within $H_{I J}$ ) for all nuclear basis functions are calculated. Each basis function can spawn new Gaussians when regions with large effective coupling $\Lambda_{J K}^{e f f}=\left|\dot{\mathbf{R}} \cdot \mathbf{d}_{J K}\right|$ (for adiabatic representation) are found. Two parameters, $\lambda_{0}$ and $\lambda_{f}$, are defined to set the limits of the region of large effective coupling. These parameters are system dependent and are defined by running test calculations. As soon as $\Lambda_{J K}^{e f f}>\lambda_{0}$, the parent basis function is classically propagated (Eqs. (21)) until the condition $\Lambda_{J K}^{e f f}<\lambda_{f}$ indicates the end of the large effective coupling region. Then, a predefined number of basis functions is evenly spawned in this region (with one of them necessarily at the point with largest $\Lambda_{J K}^{e f f}$ ). In general, the new function is spawned on a different potential energy surface, but it is also possible to spawn new functions on the same electronic surface to simulate tunneling. ${ }^{53}$ 
The spawning concept has been the inspiration for other adaptive basis set approaches as the ab initio multiple cloning based on multiconfigurational Ehrenfest (AIMC-MCE), ${ }^{60}$ which is discussed in Section 3.2.5.

In contrast to TSH, MS solutions can converge to the exact solution if an infinite basis is considered and the matrix elements are entirely computed. ${ }^{19}$ Their limitations are associated with the truncation of the basis and the use of the local approximations in the evaluation of the integrals.

\section{Recent advances in NA-MQC dynamics}

\subsection{Nonlocal effects in NA-MQC}

\subsubsection{Incorporating Decoherence}

The propagation of the semi-classical TDSE (Eq. (8)) in MFE or TSH is entirely coherent. This means that the electronic coherences - the off-diagonal terms of the density matrix $\rho_{I J}=c_{I} c_{J}^{*}$ -do not vanish during the dynamics. This problem has long been recognized ${ }^{42,61-62}$ and has been the central focus of developments in NA-MQC methods since then. It affects MFE and TSH, but not MS, which adequately addresses it. The decoherence problem has been recently reviewed by Subotnik et al. in Ref. ${ }^{29}$.

When the FSSH was proposed, it was thought that the stochastic nature of the algorithm, with each independent trajectory hopping at a different point in the phase space, would be enough to enforce decoherence over the average of the trajectories. ${ }^{43}$ Nevertheless, this stochastic effect is not sufficient ${ }^{63}$ and the overcoherence leaves clear effects on TSH results, as, for instance, in the

form of substantial divergences between the average of the electronic populations $\rho_{I I}=c_{I} c_{I}^{*}$ and the number $N_{I}$ of trajectories in each state. ${ }^{64-65}$ In other words, the equality

$$
\frac{1}{N_{\text {trajs }}} \sum_{n=1}^{N_{\text {Trajs }}} c_{I}(t) c_{I}^{*}(t)=\frac{N_{i}(t)}{N_{\text {trajs }}}
$$

which expresses the internal consistency of the algorithm, is not usually satisfied. Thus, because of the overcoherence, the nonadiabatic distribution of the trajectories deteriorates after passing multiple times through regions of significant nonadiabatic couplings. ${ }^{66}$ Decoherence corrections 
have been shown to be essential to render reliable surface hopping dynamics. ${ }^{21}$ In more fundamental terms, Ouyang and Subotnik have shown that decoherence corrections set the Poincaré recurrence time to infinity, increasing the FSSH accuracy. ${ }^{67}$ (In a different context, Bastida et al. have derived hopping algorithms constrained to satisfy Eq. $(24)^{68}$; see Section 3.2.3.)

The overcoherence is a direct effect of the nuclear localization at the classical coordinates. When the nuclear wave packet separates in different states after crossing a region of significant nonadiabatic couplings, their overlap and the nondiagonal terms of the density matrix should quickly vanish. This does not happen in MFE or TSH, where the amplitudes of the ghost states ( $c_{K}$ with $K \neq L$ ) are propagated along the same classical trajectory computed for the active state $L$. The overcoherence can also be understood as consequence of a lack of correlation in a mean field approach. ${ }^{69}$

Several ad hoc schemes have been proposed to include decoherence in each independent trajectory (see Refs. ${ }^{29,66}$ and references therein). The most straightforward treatment is to assume that decoherence is instantaneous and reset the wavefunction to

$$
\begin{aligned}
& c_{K}=0, \quad \forall K \neq L, \\
& c_{L}=1,
\end{aligned}
$$

whenever a hop to state $L$ happens. ${ }^{70}$ The instantaneous decoherence (ID) approach has been evaluated in Ref. ${ }^{65}$, where Nelson et al. show that it does not lead to internal consistency (Eq. (24) ). The ID wave-function re-setting in Eq. (25) is on the basis of more involved methods, as the augmented $\mathrm{FSSH}(\mathrm{A}-\mathrm{FSSH})^{71}$ and the decoherence-induced $\mathrm{SH}$ (DISH). ${ }^{72-73}$

Zhu, Truhlar, and co-workers have pioneered in the development of energy-based decoherence corrections (EDC), proposing a series of decay-of-mixing (DM) approaches for MFE and TSH. ${ }^{74-76}$ An approximated version of the nonlinear DM (SDM for simplified decay of mixing) approach developed by Granucci and Persico ${ }^{64}$ for TSH has become extremely popular due to its simplicity, low computational cost, and the ability to enforce internal consistency (Eq. (24)). In this approach, at each time step after integrating the semi-classical TDSE, the coefficients $c_{I}$ are corrected according to 


$$
\begin{aligned}
& c_{K}^{\text {new }}=c_{K} e^{-\Delta t / \tau_{K L}}, \quad \forall K \neq L, \\
& c_{L}^{\text {new }}=\frac{c_{L}}{\left|c_{L}\right|}\left[1-\sum_{K \neq L}\left|c_{K}^{\text {new }}\right|^{2}\right]^{1 / 2} .
\end{aligned}
$$

In these equations, $L$ is the active state and the decoherence time $\tau_{K L}$ is given by the phenomenological equation

$$
\frac{1}{\tau_{K L}^{S D M}}=\frac{\left|E_{K}-E_{L}\right|}{\hbar}\left(C+\frac{\varepsilon}{\bar{K}_{n}}\right)^{-1},
$$

where $E_{I}$ is the potential energy of state $I, \bar{K}_{n}$ is the classical kinetic energy of the nuclei. $C$ and $\varepsilon$ are parameters whose recommended values are 1 and 0.1 Hartree, respectively. ${ }^{74}$ With such values, the decoherence time for a 1-eV energy gap and 1-eV kinetic energy is approximately $1 \mathrm{fs}$. Nelson et al. ${ }^{65}$ have benchmarked the effects of the SDM (and of the original nonlinear DM) corrections to TSH, and tested the dependence on the two parameters.

While the decoherence time in Eq. (27) arose from a phenomenological analysis, a more formal derivation from the overlap evolution of frozen Gaussian wave packets have shown that this time should be proportional to the difference between the forces in different states, ${ }^{61}$ i.e.

$$
\frac{1}{\tau_{K L}^{O D C}} \propto \frac{1}{\hbar}\left|\mathbf{F}_{K}-\mathbf{F}_{L}\right|
$$

Such insight has given rise to a series of overlap-based decoherence corrections (ODC), which are based on approximated estimates of wave packet overlap decay. Granucci and Persico, ${ }^{66}$ for instance, have proposed an ODC approach dependent on two parameters, the wave packet width and the minimum overlap threshold.

The A-FSSH algorithm from Subotnik's group, in turn, propagates an auxiliary set of coordinates to estimate the overlap decay without any open parameters. ${ }^{29,63,77}$ Supposing that decoherence events can be described by a Poisson process, a stochastic algorithm is invoked to destroy the coherence of a specific state $I$ in favor of the active state $L$ according to 


$$
\begin{aligned}
& c_{K}^{\text {new }}=c_{K}, \quad \forall K \neq I, K \neq L, \\
& c_{I}^{\text {new }}=0, \\
& c_{L}^{\text {new }}=\frac{c_{L}}{\left|c_{L}\right|}\left[\left|c_{L}\right|^{2}+\left|c_{I}\right|^{2}\right]^{1 / 2} .
\end{aligned}
$$

The A-FSSH method is significantly more expensive than the traditional FSSH, and recent modifications have been proposed to speed up these calculations. ${ }^{71}$ A new development, named simultaneous FSSH (S-FSSH), improves the description of the decoherence with the explicit propagation of wave packet widths. ${ }^{78}$ Transition rates for a one-dimensional spin-boson model are benchmarked with A-FSSH and FSSH against Marcus rates in Ref. ${ }^{77}$.

This class of ODC approaches has been generalized by Gao and Thiel, ${ }^{79}$ who derived a non-Hermitian equation-of-motion (nH-EOM) approach for the full density matrix evolution, starting from the Born-Huang expansion for the molecular wavefunction (Eq. (17)) and adopting a polar form for the nuclear wavefunction. In this way, a dissipative term responsible for decoherence and proportional to the quantum nuclear momentum is naturally introduced in the TDSE. The quasiclassical limit of this method can be obtained with frozen Gaussian functions and treated in the frame of surface hopping $(\mathrm{nH}-\mathrm{SH})$. A similar approach has been derived by Ha, Lee, and Min based on an independent-trajectory approximation of the exact factorization. ${ }^{80}$

A decoherence time in the form of Eq. (28) has also been used in non-ODC approaches for NA-MQC as well, like the coherence penalty functional (CPF) for MFE ${ }^{69}$ and DISH. ${ }^{72}$ In the case of $\mathrm{CPF}$, a new term proportional to $\tau_{K L}^{-1}$ is included in the Hamiltonian, penalizing development of coherences. DISH, on its turn, innovates by using decoherence as the hop criterion.

The methods reviewed in this section rely on the independent trajectory approximation and aim at correcting the overcoherence in individual trajectories. The decoherence problem, however, can also be addressed at the ensemble level, through coupled-trajectory methods. This class of methods will be discussed later (Section 3.2.4).

\subsubsection{Incorporating Tunnelling}

One of the main challenges for NA-MQC simulations is the treatment of quantum phenomena beyond nonadiabatic effects. In particular, including tunneling has proved to be a 
challenging task. Because of their high computational cost and lack of generality, none of the existing algorithms is still in routine use.

In the context of MS, tunneling is considered by spawning new functions in the same electronic state. ${ }^{53}$ The particles are identified as tunneling, donors, and acceptors; and their identity can change during the simulations. The tunneling vectors (for all donor-acceptor combinations) are defined optimizing the system to their local minima. Tunneling thresholds (minimum donoracceptor distance) allow detecting when tunneling events can occur in analogy to the spawning threshold, while the direction of tunneling is defined using a straight-line path. When a turning point is found, the basis functions are displaced along the tunnel path. Details of the implementation and applications can be found in Refs. ${ }^{53,81}$.

A method inspired by the MS, the ab initio multiple cloning (AIMC) approach (see Section 3.2.5), considers an Ehrenfest wavefunction with the nuclear part described by a Gaussian coherent state. $^{60,82}$ Two configurations are generated to describe the bifurcation of the wave packet in the regions of strong nonadiabatic couplings. This cloning approach was recently extended to describe tunneling of hydrogen atoms, with the cloning at the turning points of the potential barrier. ${ }^{82}$

Truhlar's group proposed the army ants method to sample rare events in NA-MQC. ${ }^{83}$ The recent version of the algorithm, the army ants tunneling method, allows exploring regions of the phase space reached only by tunneling. ${ }^{84}$ It has been generalized for its use in nonadiabatic dynamic simulations in particular with Ehrenfest method, but in principle can be extended to TSH. ${ }^{85}$ The tunneling coordinate (or a combination of two) is defined using internal coordinates beforehand. So calculations need to be preceded by careful exploration of the PES. An initial ensemble of trajectories is chosen, and the probability of tunneling is calculated when a turning point is reached according to a Wentzel-Kramers-Brillouin approximation (WKB):

$$
P^{W K B}=\exp \left[\frac{-2}{\hbar} \int_{0}^{\xi_{\max }} \sqrt{2 \mu\left[\bar{E}(\mathbf{q})-\bar{E}\left(\mathbf{q}_{\mathbf{0}}\right)\right]} d \xi\right] .
$$

In this equation, $\xi$ is the distance in iso-inertial coordinates (scaled to a reduced mass $\mu$ ) at $\mathbf{q}$ with respect to the starting point $\mathbf{q}_{0}$ along the tunneling path. $\xi_{\max }$ is the length of the tunneling path. $\bar{E}$ is the mean (adiabatic or diabatic) PES. The rate of change of the coefficients $c_{J}$ (Eq. (8)) during the tunneling path are given as 


$$
\frac{d c_{J}}{d \xi}=\frac{1}{\sqrt{2 \mu^{-1}\left[\bar{E}(\mathbf{q})-\bar{E}\left(\mathbf{q}_{\mathbf{0}}\right)\right]}} \frac{d c_{J}}{d t}
$$

A probabilistic algorithm is used to decide whether the system will tunnel or not. The population of the trajectories is modulated according to the tunneling probability. Details of these algorithms can be found in Refs. ${ }^{84-85}$.

The potential of the army ants tunneling has been shown in a recent study for phenol photodissociation dynamics considering the combined effect of coherence, decoherence, and multidimensional tunneling. These simulations show the bimodal nature of the kinetic energy spectra. $^{86}$

Methods based on path integral formulation, such as ring-polymer molecular dynamics (RPMD), ${ }^{87}$ taking into account quantum behavior of quantum particles can include the effect of zero-point vibrational energy and tunneling. Recent implementations of these methods with Ehrenfest and TSH schemes for the electronic represent an alternative for the treatment of such quantum effects, as discussed below. ${ }^{88-91}$

In RPMD, quantum particles are mapped onto a closed flexible polymer of $P$ beads, profiting from an isomorphism between the quantum-statistical problem formulated in terms of a discretized version of Feynman's path integral and a classical problem. RPMD is derived for equilibrium processes, its use for non-equilibrium processes such as excited state dynamics, is done ad hoc. Sushkov, Li, and Tully ${ }^{90}$ developed a nonadiabatic version of RPMD in the frame of FSSH (RPSH). In their approach, the ring polymer is interpreted as an effective molecule moving on an effective potential energy surface coupled by NACs. With such formulation, they have proposed two different models for an effective semiclassical TDSE (Eq. (8)), which is employed for FSSH. The method is aimed at the treatment of systems with quantum and near-classical degrees of freedom and was specifically tested for computation of reaction rates with a significant contribution of tunneling.

Lu and Zhou ${ }^{88}$ have developed a conceptually different version of RPMD with TSH named path integral molecular dynamics with surface hopping (PIMD-SH). While in the RPSH the ring is treated as a molecule (each bead is an atom) that moves on a single potential energy surface, in the PIMD-SH, each bead may occupy a different state, directly related to the actual electronic 
states. The aim of the PIMD-SH method has been to sample equilibrium distributions to compute thermal averages for observables.

In Ref. ${ }^{8}$, Tavernelli derives a nonadiabatic Bohmian trajectory-based quantum dynamics (NABDY), which can treat tunneling problems. In this approach, the trajectories evolve under the action of adiabatic and nonadiabatic quantum potentials, dependent on the other trajectories, which make the dynamics exact in principle.

\subsection{New approaches to NA-MQC}

\subsubsection{Dynamics near intersections}

The nonadiabatic coupling matrix in Eq. (10) can be written as

$$
\mathbf{d}_{J K} \equiv\left\langle\psi_{J} \mid \nabla \psi_{K}\right\rangle=\frac{\left\langle\psi_{J}\left|\nabla \hat{H}_{e}\right| \psi_{K}\right\rangle}{E_{K}-E_{J}},
$$

where $E_{I}$ is the adiabatic potential energy of state $I$. Near state crossings ( $E_{K} \approx E_{J}$ ), the coupling diverges producing a steep cusp. In practical terms, the actual intersection $\left(E_{K}=E_{J}\right)$-where the coupling diverges, and the calculation of hopping probabilities breaks down - is a rare event, and usually does not pose a problem for most of the trajectories. Nevertheless, the steep shape of the nonadiabatic coupling may be missed during the trajectory propagation, if the time steps are too large (Fig. 5). ${ }^{92}$ Take, for instance, the results for $\mathrm{CNH}_{4}{ }^{+}$from Ref. ${ }^{93}$. They show that the NAC is significant in a narrow range of about $0.1 \mathrm{rad}$ around the twisted geometry. Given that the excitedstate torsional period for this molecule is about $40 \mathrm{fs}$, strong couplings are restricted to a time window of about $0.6 \mathrm{fs}$, which is of the same order as the time steps typically employed for the integration of Newton's equations $(0.1-0.5 \mathrm{fs})$.

This problem is even maximized in supramolecular assemblies, where the high density of states causes many state crossings due to localized adiabatic states lying in monomers far away in the space, and, therefore, not contributing to the electron dynamics. This type of crossing has been called trivial or unavoided crossings. ${ }^{94}$ In trivial crossings, the NAC shows an even sharper peak than usual, which in terms of dynamics translates into a substantial time localization that may be 
easily missed during numerical integration. As a result, artifacts may occur due to an improper change of diabatic character of the active state.

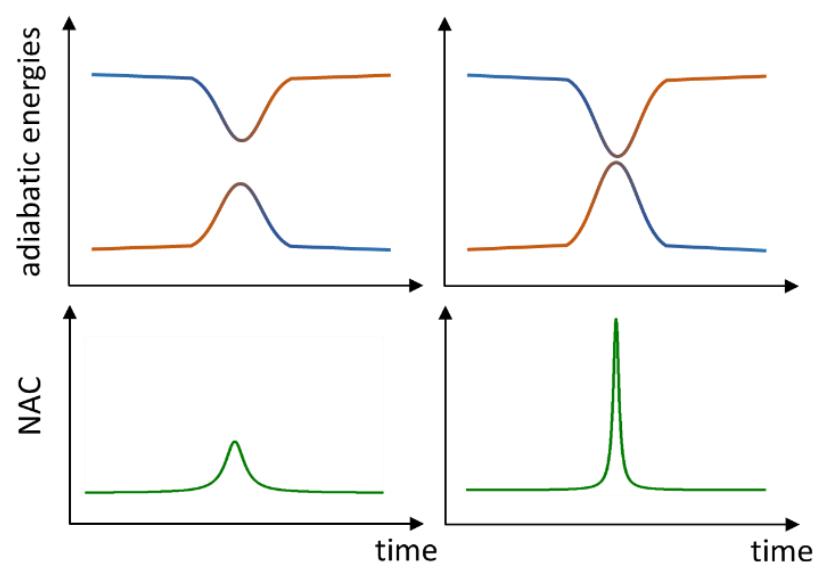

Fig. 5 Schematic illustration of the adiabatic energies and nonadiabatic couplings (NAC) as a function of time. In a weak-coupling region (right side), the width of the nonadiabatic coupling peak may be of the same order of the integration time step, $\sim 0.5$ fs.

To properly deal with NAC localization may require reducing time steps, turning the computational costs prohibitive. Alternatively, this problem has been handled with different strategies. In the context of AIMS, a method to adaptively decrease the time steps was proposed by Levine and co-workers. This algorithm keeps track of overlaps between the wavefunctions of two consecutive time steps at a reasonable computational cost. ${ }^{95}$ Spörkel and Thiel ${ }^{96}$ also developed an adaptive-step algorithm for $\mathrm{TSH}$, which propagates the trajectories with conventional time steps, but keeps track of energy conservation and orbital overlaps. When certain thresholds are surpassed, the integration takes one step back, and it is repeated with shorter time steps. Such adaptive step algorithms improve not only the description of the coupling but also minimizes instabilities caused by orbital rotations in multiconfigurational spaces.

Another strategy to deal with NAC cusps was proposed by Granucci and Persico, ${ }^{97}$ who implemented a local diabatization algorithm for TSH (LD-SH). In LD-SH, the time-dependent coefficients are not obtained by integrating Eq. (8), but through a unitary transformation

$$
\mathbf{c}(\Delta t)=\mathbf{T}^{\dagger} e^{-i \mathbf{Z} \Delta t} \mathbf{c}(0)
$$

where $\mathbf{T}$ is an adiabatic-to-diabatic transformation matrix obtained for the diabatization condition $\sigma_{J K}^{N A C}=0$. This condition implies that only NAC projections along the direction of the nuclear 
velocities are required to be null (see Eq. (10)), which renders the local character to the diabatization. In Ref. ${ }^{97}$, it is shown that the $T_{J K}$ matrix elements can be conveniently obtained from the wavefunction overlaps $S_{J K}(\Delta t)=\left\langle\psi_{J}(0) \mid \psi_{K}(\Delta t)\right\rangle$. The matrix $\mathbf{Z}$ in Eq. (33) is a simple function of the energies and diabatic Hamiltonian, which is obtained from $\mathbf{T}$. Note that the diabatization is used only to propagate Eq. (33), but the coefficients c are still defined on an adiabatic basis, and the FSSH is done in this representation. One additional advantage of the LD$\mathrm{SH}$ method is that it does not require explicit computation of NACs, as their information is already contained in T. Taking a Landau-Zener model as the standard, Plasser et al. ${ }^{98}$ showed that for weakly coupled states, LD-SH produces accurate results with time steps ten times larger than those needed to integrate Eq. (8) with the same accuracy.

Tretiak's group has also developed an algorithm to deal with trivial crossings. ${ }^{99}$ Their methodology keeps track of the overlap between electronic states in consecutive time steps (the same overlap functions $S_{J K}$ mentioned above). If the overlap exceeds a certain threshold, the crossing is considered trivial, and the hop takes place with unity probability.

The computation of the couplings $\sigma_{J K}^{N A C}$ using the norm-preserving interpolation (NPI) approach from Meek and Levine ${ }^{100}$ has been shown to account for trivial crossings as well. More details on this method are given in Section 4.3.

Wang and Prezhdo ${ }^{94}$ proposed a self-consistency (SC) check that may account for trivial crossings by simply correcting the hopping probabilities. By construction, the full FSSH probability from the active state $L$ into any state at a particular time should be ${ }^{43}$

$$
P_{L}^{\text {exact }}=\sum_{J} P_{L \rightarrow J}^{\text {exact }}=\frac{-1}{\rho_{L L}} \frac{d \rho_{L L}}{d t} .
$$

Therefore, if the coefficients $\mathbf{c}$ (or the density $\rho$ ) can be propagated accurately on a diabatic basis, then $P_{L}^{\text {exact }}$ can be computed by finite differences and compared to the sum of the actual hopping probabilities $P_{L}^{F S S H}=\sum_{J} P_{L \rightarrow J}^{F S S H}$ in Eq. (15) arising from the integration of Eq. (8). If a divergence between these probabilities is detected, it signals the occurrence of a trivial crossing. In this case, the probability for the state $I$ with the smallest energy gap to $L$ is replaced by 


$$
P_{L \rightarrow I}^{S C}=P_{L}^{\text {exact }}-\sum_{J \neq I} P_{L \rightarrow J}^{F S S H}
$$

Ref. ${ }^{94}$ claims that this SC-FSSH algorithm fixes the problem with trivial crossings leading to significant computational time savings.

\subsubsection{Niche methods}

One of the reasons for the immense success of the standard NA-MQC methods is their generality. Different from many previous nonadiabatic transition models that explicitly depend on details of the systems, as the specific topography of the crossing region, ${ }^{101} \mathrm{MFE}$, TSH, and MS require only the definition of the molecular system. There are, however, new problems for which the standard methods are not entirely tailored to deal with, but they still work as a general frame for new developments aimed at particular niches.

An example is the independent-electron SH (IESH) developed by Shenvi, Roy, and Tully ${ }^{102-104}$ to tackle the vibrational relaxation of a molecule adsorbed on a metal surface. Such a process occurs via the creation of multiple electron-hole pairs in a continuum of electronic states. The IESH approaches this problem by propagating single-electron Hamiltonians for each noninteracting electron on the metal surface, assembled as a single Slater determinant. A convenient simplification is that nonadiabatic couplings are computed as a sum of one-electron terms. With these approximations, the density matrix is calculated, and FSSH evaluated.

Another niche that has been the focus of several methodological innovations is the charge transfer between molecules within large molecular assemblies. As discussed in Section 3.2.1, the high density of states in such systems leads to problems with trivial crossings between states with electronic densities spatially far away from each other. Wang and Beljonne proposed the flexible $\mathrm{SH}$ algorithm (FSH), where TSH is applied only to a subsystem of molecules around the charge excess. ${ }^{105}$ The algorithm monitors the charge propagation to readapt the subsystem as needed. Analogous subsystem separations are also used in TSH based on QM/MM electronic structure, to avoid unphysical energy transfers between the active site and the environment. ${ }^{106}$

Still to deal with charge transfer in supramolecular assemblies, Spencer and coworkers ${ }^{107}$ developed the fragment orbital-based $\mathrm{SH}(\mathrm{FOB}-\mathrm{SH})$. In this method, the time-dependent electronic wavefunction (Eq. (7)) is written as linear combinations of site-localized wavefunctions. 
These wavefunctions are obtained from singly occupied molecular orbitals (SOMO) calculated for the isolated molecules. NACs are computed between the SOMOs and used to propagate the density matrix, allowing direct application of FSSH. Akimov has also developed a fragment molecular orbital approach in connection to $\mathrm{TSH}^{108}$ The method, based on a tight-binding extended Huckel theory and MSSH (see Section 3.2.3), has been applied to investigate systems with over 600 atoms for 5 ps.

Working on model Hamiltonians, Hammes-Schiffer and co-workers have developed a TSH methodology to study proton-coupled electron transfer (PCET) reactions in diverse media, such as solutions and interfaces with semiconductors. ${ }^{15,}$ 42, 109-111 Their approach stands out from conventional TSH applications, by the treatment of the transferring proton among the fast particles. Thus, the nonadiabatic/adiabatic branching of the process, with the proton answering to the environment's fluctuations to be guided to its final quantum state, can be simulated.

\subsubsection{Alternatives to fewest-switches probability}

Although the FSSH algorithm ${ }^{43}$ has been almost universally adopted as the standard way to obtain hopping probabilities, there are several alternatives to deal with specific problems. Stock and Thoss pointed out that it is possible to distinguish three classes of surface hopping methods. ${ }^{17}$ The first one, the quantum-classical Liouville equation (QCL) approach, comprises methods in which the partial Wigner transformed density operator in the adiabatic representation is propagated by the Quantum-Classical Liouville equation. ${ }^{2-3,18,112}$ The density evolution is written in terms of trajectories, which can switch to different adiabatic (and to averages of adiabatic) states, thanks to the nonadiabatic coupling term appearing in one of the terms of the QCL operator. We already mentioned in Section 2.2 that QCLE approaches have been used to approximately derive FSSH. ${ }^{21}$ ${ }^{48}$ A recent method in this class, the consensus surface hopping (CSH) proposed by Martens ${ }^{49}$ is discussed in more detail in Section 3.2.4.

The second class of surface hopping methods, the semiclassical approach, includes methods in which the state transition is modeled by probabilities derived from the WKB semiclassical wavefunction Ansatz, ${ }^{113-117}$ as the Landau-Zener probability for instance. ${ }^{118-120}$ In contrast to FSSH probabilities (Eq. (14)), which are instantaneous probabilities computed at each time step during the trajectory propagation, methods in the semiclassical class follow an entirely 
different philosophy. They predict transition probabilities globally after the system leaves the nonadiabatic coupling interaction region or the energy gap reaches a minimum. ${ }^{120}$ Moreover, as this class of methods does not require propagation of the TDSE, it does not suffer from decoherence problems. ${ }^{121}$

In the case of Landau-Zener TSH in adiabatic representation, a convenient way to treat the hopping probability is writing it as ${ }^{121-122}$

$$
P_{L \rightarrow J}^{L Z}=\exp \left(\frac{-\pi}{2 \hbar} \sqrt{\frac{\Delta E_{L J}\left(\mathbf{R}\left(t^{*}\right)\right)^{3}}{\left.\frac{d^{2}}{d t^{2}} \Delta E_{L J}(\mathbf{R}(t))\right|_{t=t^{*}}}}\right),
$$

where $\Delta E_{L J}$ is the adiabatic energy gap between state $L$ and $J$ evaluated at the time $t^{*}$ when it reaches its minimum value. Note that in this formulation of the Landau-Zener probability, the calculation of couplings is not necessary.

Landau-Zener theory breaks down when the collision energy becomes equal to the crossing energy. In the Zhu-Nakamura semiclassical theory, ${ }^{123}$ this problem is overcome by computing the hopping probability as

$$
P_{L \rightarrow J}^{Z N}=\exp \left[-\frac{\pi}{4 \sqrt{a^{2}}} \sqrt{\frac{2}{b^{2}+\sqrt{\left|b^{4} \pm 1\right|}}}\right]
$$

where $a$ and $b$ are functions of the diabatic forces on the two surfaces, the diabatic coupling between them, and the kinetic energy of the nuclei (see Ref. ${ }^{116}$ for explicit formulas).

Historically, semiclassical probability methods were developed first, as a direct application of perturbation theory. ${ }^{101}$ A recent benchmark comparing FSSH and Zhu-Nakamura TSH for cistrans azobenzene photoisomerization showed that both methods yield equivalent results. ${ }^{117}$ Benchmark comparison between FSSH and Landau-Zener TSH for a two-dimensions/three-states model system also revealed a good agreement between the two methods. ${ }^{121}$ Such agreement in both cases is not completely surprising, as the crossing regions in these examples can be well represented by the linear-crossing topography for which the Landau-Zener model was derived. 
Nevertheless, as semiclassical probabilities are usually derived for specific crossing topographies, ${ }^{101}$ they may not be entirely adequate to be employed in general NA-MQC methods.

The third class of surface hopping, the quasi-classical approach, includes methods in which the state transition probability is modeled by a local approximation of the TDSE, ${ }^{124-127}$ as in the FSSH itself. Recent methodological extensions to account for time-dependent fields and spin-orbit couplings fall within this category. ${ }^{128-129}$ They are discussed in Section. 3.3.

Still in this latter class, Bastida et al. ${ }^{68}$ derived two hopping probability models to fulfill internal consistency (Eq. (24)). The first model is based on collective probabilities (CP) and depends on the fraction of trajectories in the initial and target states, moving beyond the independent trajectory approximation (Section 3.2.4). The second model, named independent probabilities (IP) algorithm, imposes internal consistency for independent trajectories, leading to the hopping probability

$$
P_{L \rightarrow J}^{I P}=c_{J}(t+\Delta t)
$$

They showed, however, that the IP algorithm is computationally inefficient, requiring a large number of trajectories to converge. More recently, Akimov et al. ${ }^{130}$ rederived the IP algorithm in the context of Markovian processes in their Markov State Surface Hopping (MSSH). The MSSH (or IP) approach has been shown to outperform FSSH for a three-state superexchange model (involving probability transfer between non-directly coupled states), delivering probabilities in better agreement with the exact results. ${ }^{130}$

Wang, Trivedi, and Prezhdo ${ }^{131}$ developed the global flux SH (GFSH), which differs from the standard FSSH only in the way the probability is computed. Instead of using Eq. (14), in GFSH the states are split into two groups, those in which the population increased between two timesteps (group A, with $\Delta \rho_{I I}=\rho_{I I}(t+\Delta t)-\rho_{I I}(t)>0$ ) and those in which the population reduced (group $\mathrm{B}, \Delta \rho_{J J}<0$ ). Then, the population flow balance between the two groups allows defining the hopping probability from the active state $L$ belonging to group A to a state in group B as

$$
P_{L \rightarrow J}^{G F S H}=\frac{\Delta \rho_{J J}}{\rho_{J J}} \frac{\Delta \rho_{L L}}{\sum_{K \in A} \Delta \rho_{K K}} \quad(L \in A ; J \in B)
$$


The GFSH was also formulated to simulate super-exchange phenomena, as it occurs in singlet fission and Auger processes.

\subsubsection{Coupled trajectories}

The independent trajectory approximation has been fundamental to the success of TSH and MFE, allowing for their computational efficiency and straightforward on-the-fly implementation. This approximation, however, is the reason of some of the main handicaps of these methods, as the overcoherence discussed in Section 3.1.1. Several algorithms to coupled trajectories but still in the frame of on-the-fly propagation have been developed. An example is the coupled probabilities algorithm (CP) from Bastida et al., ${ }^{68}$ in which hopping probabilities explicitly depend on the fraction of trajectories in each state, enforcing internal consistency.

The second-quantized surface hopping (SQUASH) ${ }^{132}$ is a multi-trajectory version of TSH, allowing energy transfer between trajectories, but requiring energy conservation at the ensemble level. In this way, it aims at emulating a wave packet propagation. The SQUASH formalism is completely analogous to that of FSSH but generalized to an $N$-particles (or trajectories) formulation, each one following an independent semiclassical TDSE. Thus, hopping probabilities (Eq. (14)) are not computed using the usual coefficients $c_{J}^{(n)}$ for the electronic state $J_{n}$ of trajectory $n$ but using $N$-trajectory coefficients $C_{\gamma}=c_{J_{1}}^{(1)} c_{J_{2}}^{(2)} \cdots c_{J_{N}}^{(N)}$ for a state $\gamma$ defined by the state occupations of all trajectories $\left(J_{1}, J_{2}, \cdots, J_{N}\right)$. The local TDSE in SQUASH differs from that in Eq. (8) by considering the nuclear kinetic energy term. Strictly speaking, SQUASH is not a coupled-trajectory method, as each trajectory is still propagated independently. Nevertheless, there is a flow of information between trajectories, first, because of the $N$-trajectory character of the states and, second, because the energies of the $N$-trajectory states are used to decide about the hop rejection and momentum rescaling. For a single-trajectory state, SQUASH reduces to conventional FSSH. Some effects beyond FSSH are already recovered at the two-trajectory states level. ${ }^{133}$

Another method defining the hoping based on the trajectory ensemble is the consensus surface hopping (CSH) proposed by Martens. ${ }^{49}$ In CSH, phase space populations and coherences are employed to propagate a set of coupled equations for the mixed-quantum-classical limit of the 
Liouville equation (QCLE). ${ }^{1}$ The state occupied by a certain trajectory at time $t$ is stochastically updated between time steps according to the probability

$$
P_{L \rightarrow J}^{C S H}=\frac{-2 \Delta t}{\left\langle\rho_{J J}(\overline{\mathbf{R}}, \overline{\mathbf{P}})\right\rangle_{N}} \sigma_{L J}^{N A C}(\overline{\mathbf{R}}, \overline{\mathbf{P}})\left\langle\operatorname{Re}\left(\rho_{L J}(\overline{\mathbf{R}}, \overline{\mathbf{P}})\right)\right\rangle_{N}
$$

This expression for the adiabatic representation is analogous to the FSSH probability (Eq. (15)) but with the critical difference that the populations and coherences at the classical phase space point $(\overline{\mathbf{R}}, \overline{\mathbf{P}})$ are computed as a mean value over all $N$ trajectories. For this reason, all trajectories are effectively coupled to each other. As in SQUASH, no energy conservation is imposed for individual trajectories, as it should be conserved only for the ensemble. Moreover, no decoherence correction is needed, as the evolution of the coherences is explicitly accounted for.

The usual approach to solving the TDSE for the full molecular systems starts from the Born-Huang expansion of the total molecular wavefunction given in Eq. (17). ${ }^{134}$ Abedi, Maitra, and Gross have proposed, instead, a new time-dependent formalism denominated exact factorization $(\mathrm{EF}) .{ }^{135-136}$ Based on Hunter's work on the time-independent case, ${ }^{137}$ they proved that the total wavefunction of a molecule can be exactly factorized as

$$
\Phi(\mathbf{r s}, \mathbf{R} \boldsymbol{\sigma}, t)=\chi(\mathbf{R} \boldsymbol{\sigma}, t) \Psi_{\mathbf{R}}(\mathbf{r s}, t)
$$

where $\Psi_{\mathbf{R}}(\mathbf{r s}, t)$ satisfies the partial normalization condition

$$
\sum_{\mathbf{s}} \int d \mathbf{r}\left|\Psi_{\mathbf{R}}(\mathbf{r s}, t)\right|^{2}=1
$$

(Nuclear $\sigma$ and electronic $\mathbf{s}$ spin coordinates are explicitly indicated.) $\Psi_{\mathbf{R}}(\mathbf{r s}, t)$ can be interpreted as a conditional probability parametrically depending on the nuclear coordinates $\mathbf{R}$, while $\chi(\mathbf{R} \boldsymbol{\sigma}, t)$ is a marginal probability for the nuclear coordinates. The wavefunctions in Eq. (41) are unique within a phase-dependent component and can also be identified as the nuclear $(\chi)$ and electronic $(\Psi)$ wavefunctions. Within the EF framework, nuclei and electrons can be propagated quantum mechanically by a set of coupled equations for $\chi$ and $\Psi$, whose equationsof-motion depend on a time-dependent potential energy surface (TDPES) and on a time-dependent potential vector. 
The EF provides a framework to analyze the nuclear-electron coupling within static and dynamical approaches, ${ }^{138-143}$ allowing the definition of new NA-MQC dynamics methods. ${ }^{144-145}$ This is the case of the recently developed coupled-trajectory MQC (CT-MQC), ${ }^{10,146-148}$ which shares some similarities with the traditional Ehrenfest method. Starting from the expansion in the adiabatic states of the electronic wavefunction given by Eq. (7), the equation-of-motion for the time-dependent coefficients becomes

$$
\frac{d c_{J}^{E F}}{d t}=\frac{d c_{J}^{M F E}}{d t}+\frac{d c_{J}^{Q M}}{d t}
$$

where $\dot{c}_{J}^{M F E}$ is given by the standard MFE quantum EOM (Eq. (8)) and $\dot{c}_{J}^{Q M}$ is a correction coming from the EF model, which depends on the quantum momentum $\mathbf{Q}$ (see Eq. S17 of the Supporting Information of Ref. ${ }^{147}$ for the definition of this quantity) and on the adiabatic impulse $\mathbf{f}$ ( $\left.\mathbf{f}=-\int^{t} d t^{\prime} \nabla H_{K K}\right)$. The force acting on the nuclei are analogously written as

$$
\mathbf{F}^{E F}=\mathbf{F}^{M F E}+\mathbf{F}^{Q M},
$$

where $\mathbf{F}^{M F E}$ is the mean-field force given by Eq. (12) and $\mathbf{F}^{Q M}$ is the EF correction, also dependent on $\mathbf{Q}$ and $\mathbf{f}$. A core feature of the CT-MQC method is that the evaluation of the quantum momentum $\mathbf{Q}$ along a trajectory depends on the nuclear positions in all other trajectories at the same time step. ${ }^{10}$

Gorshkov, Tretiak, and Mozyrsky have developed a semiclassical Monte-Carlo (SCMC) approach, which post-process a conventional TSH result to obtain fully correlated results. ${ }^{149}$ In their method, they first use a path integral formalism to get an expression to the nuclear wavefunctions (for a specific electronic state) in powers of nonadiabatic couplings. This procedure results in a convoluted general formula for the probability of the electrons occupy a given state at time $t$. This probability, corresponding to a double path integral in the subspace of electronic states, is then computed by using a conventional TSH simulation to sample the space for a Monte Carlo integration. Although the SCMC method opens new perspectives for high-level NA-MQC dynamics, it is still unpractical due to its high computational costs, being restricted so far to tests on model systems. Implementation of on-the-fly branching of the wavepackets ${ }^{150}$ is the first step toward reducing the underlying numerical effort in the SCMC approach. 


\subsubsection{Trajectory-guided Gaussian methods}

Trajectory-guided Gaussian methods form a class of methods that model the nuclear wave packet time evolution by frozen Gaussians centered at classical trajectories. Such methods remount to the works of Heller ${ }^{151}$ and those of Herman and $\mathrm{Kluk}^{46}$ on semiclassical frozen Gaussians in the early 1980s. Their advantage in comparison to the full quantum propagation of the nuclear wave packet $^{152-153}$ is that, due to the constraint to follow a classical trajectory (which at each time step is only a hyper point in the phase space), they can be adapted to on-the-fly protocols, not necessarily requiring pre-computed potential energy surfaces.

A series of methods have adopted the trajectory-guided Gaussian concept as a strategy to collect information on decoherence to correct NA-MQC dynamics. This is the case of the overlapbased decoherence corrected (ODC) methods discussed in Section 3.1.1. These methods, however, do not truly propagate the nuclei as Gaussian wave packets, but instead, use short-term auxiliary expansions to estimate the overlap dissipation between trajectories evolving in different electronic states.

Among the methods in which nuclei are really propagated by trajectory-guided Gaussians, the most well-known is the multiple spawning (MS), ${ }^{6}$ discussed in Section 2.3.

Recent developments in this class of methods include the Multiconfigurational Ehrenfest (MCE), ${ }^{59,} 154$ where the molecular wavefunction Ansatz is generalized from the single configuration given by Eq. (3), into a linear combination of configurations. MCE is closely related to MS. ${ }^{154}$ The molecular wavefunction is also expanded on a set trajectory basis functions (TBF) composed of electronic and nuclear parts. The nuclear parts are written as generalized Gaussians. Nevertheless, different from MS, in which Gaussian centers are propagated on a single potential energy surface, in MCE, they are propagated on a mean field. The comparison between MCE and decoherence-corrected TSH suggests that MCE can naturally account for decoherence. ${ }^{22}$

A new development of MCE named ab initio multiple cloning (AIMC-MCE) ${ }^{60}$ includes the cloning of Ehrenfest configurations into two, one of which is then guided by a single PES, while the other is guided by the mean-field force for the remaining states. With such cloning procedure, the method can describe the bifurcation of the wavefunction after leaving the strong nonadiabatic coupling region and tunneling. ${ }^{82}$ 
Other Gaussian-guided methods are the Kondorskiy-Nakamura model, ${ }^{155}$ the quantum trajectories on Gaussian basis (QTGB), ${ }^{156-157}$ and the semiclassical Monte-Carlo (SCMC) approach. ${ }^{149}$ The former merges the Kluk-Herman semi-classical frozen Gaussian method with the Zhu-Nakamura theory of nonadiabatic transitions (see Section 3.2.3 for information on the ZhuNakamura method). QTGB employs Gaussian-guided quantum trajectories to overcome the limitations of computing the quantum potential in Bohmian dynamics. SCMC is discussed in Section 3.2.4.

A compelling framework for the trajectory-guided Gaussian methods has been provided by the Gaussian-based multiconfigurational time-dependent Hartree (G-MCTDH) approach. ${ }^{158}$ The full MCTDH method ${ }^{152-153}$ can, in principle, provide the exact full-quantum mechanical nonadiabatic wave packet propagation of a molecular system with $N_{f}$ nuclear degrees of freedom. It is based on a multiconfigurational Ansatz for the nuclear wavefunction with general form ${ }^{134}$

$$
\chi_{K}(\mathbf{R}, t)=\sum_{m} A_{K}^{m}(t) \xi^{m}\left(R_{1}, R_{2}, \ldots, t\right),
$$

where each configuration $\xi^{m}$ is given in terms of a Hartree product of functions $\phi$ of single nuclear coordinates $R_{q}$. In G-MCTDH, this Hartree product is split into two subsets, those nuclear coordinates that will be treated by full quantum mechanics (as in the full MCTDH), and those that will be dealt with approximately, using Gaussian functions $g$ (either frozen or thawed): ${ }^{153}$

$$
\xi^{m}=\prod_{q=1}^{p} \phi_{q}^{m} \prod_{q=p+1}^{N_{f}} g_{q}^{m} .
$$

In the limit that all $N_{f}$ nuclear degrees of freedom are treated by Gaussian functions, the method is named variational multiconfigurational Gaussian wave packet (vMCG). In this case, the timedependent coefficients $A_{K}^{m}$ in Eq. (45) are propagated by the same EOM as in multiple spawning, Eq. (22), but without the constraint of following classical trajectories. Then, a series of approximations on the integrals contained in EOM may be adopted to control the quantum level, from full global to local approximations. ${ }^{159}$ In the local limit, the method reduces to the direct dynamics trajectory-guided frozen Gaussian level (DD-vMCG). Thus, the G-MCTDH approach can be used to test the effect of various approximations on nonadiabatic dynamics hierarchically. A comparative analysis of vMCG, MS, and TSH is made in Ref. ${ }^{19}$. The formal connection between vMCG and MFE is discussed in Ref. ${ }^{20}$. 


\subsubsection{Slow and rare events}

Computational cost is usually the primary constraint limiting on-the-fly NA-MQC simulations. In the particular case of phenomena involving rare events (requiring thousands of trajectories) or events taking place in long time range (requiring propagation for hundreds of picoseconds or more), it may be unfeasible to resort to dynamics, and reaction rate theory may still be the best option. ${ }^{160-162}$ Nevertheless, there are various algorithms that may help speed-up or extend the range of applicability of NA-MQC. This is the case, for instance, of the army ants method to sample rare events with MFE and TSH. ${ }^{83}$ Another example is the use of Hessians to integrate the classical EOM with large time steps, as developed in the 1990s by Helgaker and coworkers ${ }^{163}$ and recently implemented in an MFE approach. ${ }^{38}$

In Ref. ${ }^{164}$, a TSH approach inspired in the meta-dynamics ${ }^{165}$ is proposed to deal with slow or rare events. Named metasurface-hopping (MSH), it works on biased sampling to speed-up the data acquisition, which are later corrected to deliver the unbiased results. The basic idea is to perform biased TSH dynamics by speeding up the transitions with a scaled NAC

$$
\mathbf{d}_{J K}^{\text {biased }}(t)=\kappa \mathbf{d}_{J K}(t)
$$

where $\kappa$ is a time-independent scaling factor $(\kappa>1)$. MSH has explicitly been formatted to deliver ensemble-averaged reaction rates $\Gamma$ derived from the Fermi's golden rule. Because these rates are proportional to $\mathbf{d}_{J K}^{2}$, the relation between the biased and unbiased ensemble-averaged rates is simply

$$
\left\langle\Gamma^{\text {biased }}\right\rangle \approx \kappa^{2}\left\langle\Gamma^{\text {unbiased }}\right\rangle
$$

Nijamudheen and Akimov ${ }^{166}$ pointed out that the scaling relation in Eq. (47) is rigorously valid only for a two-level system. For a more general case, they propose to run the dynamics with several values of $\kappa$, and then fit the time constants obtained from these simulations according to

$$
\tau(\kappa)=\frac{1}{\kappa^{2} A+B}
$$

After getting $A$ and $B$, the unbiased time constant can be obtained merely by making $\kappa=1$ in Eq. (49). This procedure has been called accelerated nonadiabatic dynamics (X-NA-MD). 
During dynamics, the vibronic Hamiltonian $\left[H_{J K}-i \hbar \sigma_{J K}^{N A C}\right]$ in Eq. (8) fluctuates randomly around some average values. Based on this observation, Akimov has also recently proposed to adopt a quasi-Stochastic Hamiltonian for longer dynamics in condensed-matter systems, sampling Hamiltonian from the short-time dynamics, getting frequencies and amplitudes, and recovering the Hamiltonian for the indefinite time. ${ }^{167}$

\subsection{NA-MQC: beyond internal conversion}

\subsubsection{Intersystem crossing}

NA-MQC methods were initially developed to deal with internal conversion (IC) processes, the fastest nonadiabatic processes known. Nevertheless, spin-orbit coupling (SOC) inducing transitions between states of different multiplicities, may also be relevant on short timescales. ${ }^{168}$ For this reason, in the last few years, the incorporation of SOC into NA-MQC schemes to simulate intersystem crossing (ISC) dynamics has become an active area of research. ${ }^{129,169-174}$

In NA-MQC, SOC is usually included by considering the coupling term

$$
\sigma_{J K}^{S O C}=\frac{i}{\hbar}\left\langle\psi_{J}\left|\hat{H}^{S O C}\right| \psi_{K}\right\rangle
$$

where $\hat{H}^{S O C}$ is the perturbative contribution to the electronic Hamiltonian $\hat{H}^{T O T}=\hat{H}_{e}+\hat{H}^{S O C}$. Several investigations in this field worked the spin-diabatic (sd) representation, where the electronic wavefunctions are spin-eigenstates. ${ }^{115,171,173,175-177}$ (This spin-diabatic representation is simply the conventional adiabatic representation commonly used in quantum chemistry; see Fig. 6.) SOC modifies the nondiagonal terms, but energies and forces are evaluated for the unperturbed PES. In these applications, ISC TSH has been simulated either using Landau-Zener theory ${ }^{115}$ or through a direct application of the FSSH algorithm, replacing $\sigma_{J K}^{N A C}$ by $\sigma_{J K}^{T O T}=\sigma_{J K}^{N A C}+\sigma_{J K}^{S O C}$ in Eq. (8). ${ }^{171,173,175}$ 


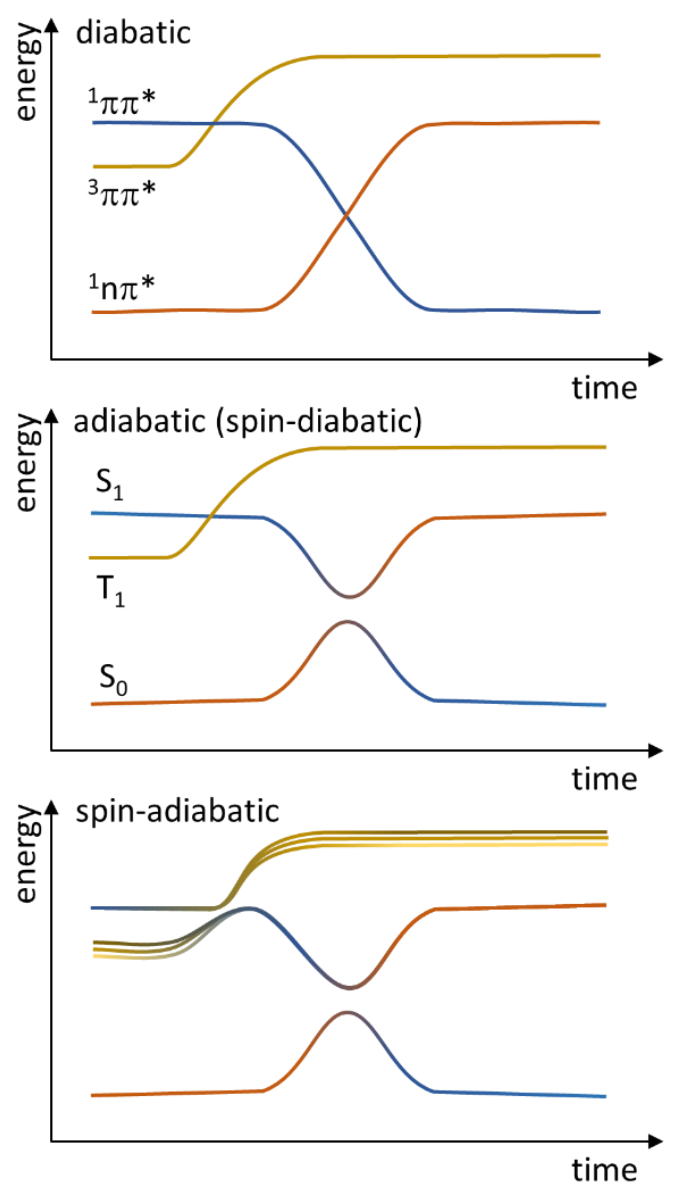

Fig. 6 Schematic illustration of the time evolution of the potential energies in different representations as a function of time. Top: diabatic representation. The nonadiabatic coupling between any pair of states is null. Each state is characterized by the same electronic type of density and multiplicity at all times (e.g., ${ }^{1} \mathrm{n} \pi^{*},{ }^{1} \pi \pi^{*}$, etc.). Middle: adiabatic (or spin-diabatic, or still molecular Coulomb Hamiltonian ${ }^{169}$ ) representation. States of different multiplicities have zero nonadiabatic couplings (e.g. $T_{1}$ and $S_{1}$ ). The electronic character may change during time. Bottom: spin-adiabatic (or diagonal, or fully adiabatic) representation. Every pair of states may be nonadiabatically coupled. The electronic and spin characters of the states may change with time. Triplet states split into three components corresponding to $M_{s}=0,+1$, and -1 in the adiabatic representation. The split is not shown at the correct scale in the figure.

Granucci and Persico have shown, however, that the spin-diabatic representation poses some severe challenges to control the SOC phases during the dynamics, which usually results in wrong hopping probabilities. ${ }^{172}$ In contrast, these problems are not present if dynamics is propagated in a spin-adiabatic (sa) representation (Fig. 6). This representation considers the $H^{\text {TOT }}$ eigenstates, where $\sigma_{L K}^{S O C}=0$ and the effect of SOC is included in the energies. It involves the diagonalization of the Hamiltonian matrix

$$
\hat{\mathbf{H}}^{T O T, s a}=\mathbf{U}^{\dagger} \hat{\mathbf{H}}^{T O T, s d} \mathbf{U},
$$


sorting out the problems with phases and rotational invariance.

In the spin-adiabatic representation, the quantum EOM (Eq. (8)) can be rewritten using the matrix representation

$$
\frac{d \mathbf{c}^{s a}}{d t}=-\mathbf{U}^{\dagger}\left(\frac{i}{\hbar} \mathbf{H}^{s d}+\boldsymbol{\sigma}^{T O T, s d}\right) \mathbf{U} \mathbf{c}^{s a}-\mathbf{U}^{\dagger} \frac{d \mathbf{U}}{d t} \mathbf{c}^{s a} .
$$

The evaluation of the time derivative of the transformation matrix ( $\dot{\mathbf{U}}$ ) is technically challenging ${ }^{169}$ and in the SHARC (surface-hopping in adiabatic representation including arbitrary couplings) approach from González's group, a 3-step integrator approach is proposed to avoid evaluating these terms: ${ }^{129,}, 178$

$$
\mathbf{c}^{s a}(t) \stackrel{\mathbf{U}}{\longrightarrow} \mathbf{c}^{s d}(t) \stackrel{\Delta t}{\longrightarrow} \mathbf{c}^{s d}(t+\Delta t) \stackrel{\mathbf{U}^{\dagger}}{\longrightarrow} \mathbf{c}^{s a}(t+\Delta t) .
$$

Thus, the time-dependent coefficients are propagated on the spin-diabatic basis and transformed back to the spin-adiabatic basis for the calculation of the hopping probabilities. A variant of the 3step integrator has been recently implemented by Perderzoli and Pittner, ${ }^{178}$ with specific phase control. In the same paper, they present another method with explicit treatment of $\dot{\mathbf{U}}$.

To avoid that $\dot{\mathbf{U}}$ appear explicitly in the FSSH probabilities, the hopping probabilities are not directly calculated with Eq. (15). Instead, they rely on a variant of the FSSH formula first derived in Ref. ${ }^{97}$ :

$$
P_{L \rightarrow J}^{F S S H \bmod }=\max \left[0,\left(1-\frac{\left|c_{L}^{s a}(t+\Delta t)\right|^{2}}{\left|c_{L}^{s a}(t)\right|^{2}}\right) \frac{\operatorname{Re}\left(c_{J}^{s a}(t+\Delta t) P_{J L}^{s a^{*}} c_{L}^{s a^{*}}(t)\right)}{\left|c_{L}^{s a}(t)\right|^{2}-\operatorname{Re}\left(c_{L}^{s a}(t+\Delta t) P_{L L}^{s a^{*}} c_{L}^{s a^{*}}(t)\right)}\right]
$$

where $\mathbf{P}$ is the time-propagator matrix $\mathbf{c}^{s a}(t+\Delta t)=\mathbf{P}^{s a}(t+\Delta t, t) \mathbf{c}^{s a}(t)$, given by $\mathbf{P}^{s a}(t+\Delta t, t)=\mathbf{U}^{\dagger}(t+\Delta t) \mathbf{P}^{s d}(t+\Delta t, t) \mathbf{U}(t)$. The propagator in the spin-diabatic representation is simply $\mathbf{P}^{s d}=\exp \left(-\left(i \hbar^{-1} \mathbf{H}^{T O T, s d}+\boldsymbol{\sigma}^{T O T, s d}\right) \Delta t\right)$. (Note that Eq. (53) is not restricted to ISC problems and may be used for conventional FSSH as well, as it was first done in Ref. ${ }^{97}$.)

Spin-orbit couplings also impact the propagation of the classical EOM (Eq. (13)), whose forces should be computed in the spin-adiabatic representation, where they are given as 


$$
\mathbf{F}_{L}^{s a}=-\nabla_{\alpha} E_{L}^{s a}=-\sum_{J K} U_{J L}^{*} U_{K L}\left(\left\langle\psi_{J}\left|\nabla_{\alpha} H^{s d}\right| \psi_{K}\right\rangle-i \hbar \nabla_{\alpha} \sigma_{J K}^{S O C}\right),
$$

A practical limitation to apply Eq. (54) is that the gradient of the spin-orbit coupling $\left(\nabla \boldsymbol{\sigma}^{S O C}\right)$ is not usually available in quantum chemistry programs. For this reason, this quantity is routinely neglected ${ }^{169,178}$ :

$$
\nabla_{\alpha} \sigma_{J K}^{S O C} \approx 0
$$

rendering the forces

$$
\mathbf{F}_{L}^{s a} \approx-\sum_{J K} U_{J L}^{*}\left[\delta_{J K} \nabla_{\alpha} E_{J}^{s d}+\left(E_{K}^{s d}-E_{J}^{s d}\right) \mathbf{d}_{J K, \alpha}^{s d}\right] U_{K L}
$$

where $\mathbf{d}$ is the nonadiabatic coupling vector defined in Eq. (11). Full calculation of the forces through Eq. (54) has been implemented in the semiempirical FOMO-CI method by Granucci and Persico. ${ }^{172}$

Although the three-step integrator pioneered by the SHARC approach ${ }^{129}$ has represented an advance in comparison to the earliest simulations based spin-diabatic representations, the impact of the underlying approximations is still not completely clear. An exemplary case is benzophenone in the gas phase, whose transfer from the singlet to the triplet manifold is 5 ps, as experimentally determined by time-resolved photoelectron spectroscopy. ${ }^{179}$ While TSH simulations on a spin-adiabatic basis using the semiempirical FOMO-CI method predicted a time constant of 6 ps, ${ }^{180}$ TSH simulations with SHARC using CASSCF delivered an artificially fast time constant, with $S_{1}$ disappearing within 0.7 ps. ${ }^{181}$ It is possible that the reason for the difference resides on the different electronic structure methods (FOMO-CI x CASSCF) rather than on the basis transformation; a comparison of these methods based on the same electronic structure would be welcome to shed light on this point.

MS methods have also been recently generalized to consider ISC events based on the spindiabatic representation. ${ }^{176-177}$ In the method called Generalized Ab initio Multiple Spawning (GAIMS), the equations of motions were modified, including SOC along nonadiabatic couplings into effective NAC terms. As discussed in Ref. ${ }^{176}$, the problems to control the SOC phase, which severely affect TSH, does not occur in GAIMS. 


\subsubsection{External fields}

The interaction with external fields has also been addressed in the context of NA-MQC dynamics. $^{73}$, 128-129, 178, 182-186 The Hamiltonian for the radiation-matter interaction (or electromagnetic coupling, EMC) is given as ${ }^{184}$

$$
\hat{H}^{E M C}=-\frac{e}{2 m_{e} c} \sum_{i} \mathbf{A}\left(\mathbf{r}_{i}, t\right) \cdot \hat{\mathbf{p}}_{i}
$$

where $\mathrm{A}(\mathbf{r}, t)$ is the vector potential of the electromagnetic field, $\hat{\mathbf{p}}_{i}$ is the momentum operator of electron $i$, and the sum runs over all electrons with coordinate $\mathbf{r}_{i} \cdot e, m_{e}$, and $c$ are the electron charge, the electron mass, and the speed of light. In the dipole approximation, it results in a coupling between states $K$ and $L$ given by

$$
\sigma_{L K}^{E M C}=-\frac{i}{\hbar} \boldsymbol{\mu}_{L K} \cdot \mathbf{E}(t)
$$

where $\boldsymbol{\mu}_{L K}$ is the electric transition dipole matrix element and $\mathbf{E}(t)$ the time-dependent electric field $(\mathbf{E}=-\dot{\mathbf{A}} / c)$.

Working in the semiclassical limit of the quantum Liouville-von Neumann equation, Mitrić et al. ${ }^{128}$ showed that, for a pure initial state and neglecting dissipative effects, the propagation of Wigner functions in the phase space is equivalent to the semi-classical TDSE in Eq. (8) with $\sigma_{J K}^{E M C}$ replaced for $\sigma_{J K}^{N A C}$. Thus, FSSH including radiation-matter interactions can be directly done, in a method they have named field-induced surface hopping (FISH). ${ }^{128,187}$ If internal conversion is also allowed, the semiclassical TDSE will contain both coupling terms $\sigma_{J K}^{N A C}+\sigma_{J K}^{E M C}$ .184

FISH has been recently extended to take into account nonlinear field effects up to $\boldsymbol{\alpha E}^{2}$, where $\boldsymbol{\alpha}$ is the polarizability. ${ }^{188}$ This is accomplished by first separating the ensemble of electronic states into two subgroups, essential and nonessential, according to their response to the field. With this separation, a quadratic correction

$$
\sigma_{J K}^{E M C(2)}=-\frac{i}{4 \hbar^{2}} \mathbf{E}^{T} \boldsymbol{\alpha}_{J K} \mathbf{E}
$$


is added to $\sigma_{J K}^{E M C}$, with $\boldsymbol{\alpha}_{J K}$ written in terms of the transition dipole matrices between essential and nonessential states.

The FISH approach does not consider the effect of the field on the potential energy surfaces, only on the nonadiabatic transitions. In Ref. ${ }^{189}$, Bajo and coworkers discuss how for strong fields, field-induced changes in the gradients should also be taken into account for a proper treatment of the dynamics. Using the SHARC approach discussed above in Section 3.3.1, which explicitly includes the field effects on the potentials, they showed that NA-MQC simulations might deliver an excellent agreement with full quantum results.

The coupling between molecular electronic states and those of an electromagnetic field confined within a cavity has been recently addressed in Ref. ${ }^{190}$, in the context of surface hopping simulations. Different from previous applications, in which the field only disturbed the electronic states, in this novel approach, the dynamics is propagated in a basis of polariton states arising from the molecule/field coupling in the first order in the electric field. Simulations based on QM/MM TSH have been performed for up to 1600 Rhodamine chromophores within the cavity.

External fields have been included in MS as well. In the External Field Ab initio Multiple Spawning (XFAIMS) method, ${ }^{191}$ Eq. (22) is modified to include the radiation-matter interaction in the $H_{J K}$ term of the Hamiltonian, still in the dipole approximation. In the coupling region, new trajectory basis functions are spawned when the field reaches an extreme, which may happen few times for long pulses.

\subsubsection{General couplings}

We have discussed in the last two sections that although the standard NA-MQC methods have been first derived for internal conversion, other kinds of couplings inducing nonadiabatic transitions between electronic states, as spin-orbit couplings or time-dependent electric field interactions, may be considered without changing the formalism substantially. (In the case of SOC and strong fields, however, a change of representation may be required as discussed in Sections 3.3.1 and 3.3.2.)

In principle, dynamics based on a general coupling like

$$
\sigma_{J K}^{T O T}=\sigma_{J K}^{N A C}+\sigma_{J K}^{S O C}+\sigma_{J K}^{E M C}+\sigma_{J K}^{E M C(2)}+\ldots
$$


would allow monitoring the real-time competition between diverse nonadiabatic processes. Such flexibility towards different couplings has been explored to develop general methods tailored for such arbitrary couplings. This philosophy is on the basis of at least two NA-MQC implementations, SHARC (which clearly states this idea already in its name explicitly mentioning

arbitrary couplings) ${ }^{129,169}$ and PYXAID. ${ }^{73}$ Recent developments by Martínez, Curchod, and coworkers indicate that MS will progress in the same direction. ${ }^{82,176,191}$

Although this arbitrary-coupling philosophy has been opening new research possibilities, it is still unclear how general, such approach may really be. Typically, different nonadiabatic interactions work on different time scales. To simulate the full dynamics at once, from few femtoseconds of EM interactions, through the few picoseconds of IC, to the nanoseconds of ISC may result unpractical. Thus, these methods for arbitrary couplings are tailored not for general, but to particular problems, where various nonadiabatic interactions tend to compete on the same timescale.

\section{Electronic structure for NA-MQC dynamics}

The overall tendency of NA-MQC dynamics is to couple the dynamics method to an electronic structure method, which can provide the key ingredients for the time evolution, the potential energies of the ground and excited electronic states $\left(E_{J}\right)$, the gradients of these energies $\left(\mathbf{G}_{J}\right)$, and the nonadiabatic couplings $\left(\mathbf{d}_{J K}\right)$ between pair of states:

$$
\begin{aligned}
& E_{J}=\left\langle\psi_{J}\left|\hat{H}_{e}\right| \psi_{J}\right\rangle, \\
& \mathbf{G}_{J}=\nabla E_{J}, \\
& \mathbf{d}_{J K}=\left\langle\psi_{J} \mid \nabla \psi_{K}\right\rangle .
\end{aligned}
$$

All these quantities are computed for specific nuclear geometries, in general, dictated by classical trajectories. Depending on the process and properties investigated, other quantities like transition dipole moments and spin-orbit couplings may be needed as well. Alternatively, the $\mathbf{d}_{J K}$ vectors may be replaced by $\sigma_{J K}^{N A C}$ (see Eq. (10)).

The calculation of these quantities is the computational bottleneck of the on-the-fly NAMQC propagation. Moreover, their quality is the main determinant of the dynamics accuracy. In this section, we survey the main electronic structure methods that have been used for NA-MQC 
dynamics, highlight the pros and cons of each one, and pointing out potential relevant developments. We also separately discuss the computation of couplings in Section 4.3, which is often treated as a post-processing of the electronic structure data. Later, in Section 7, we return to the accuracy problem. NA-MQC dynamics has often been run in association with hybrid methods, ${ }^{13}$ most notably quantum-mechanics/molecular-mechanics (QM/MM). ${ }^{32,}{ }^{192}$ The methodological extension into hybrid methods is straightforward, and it is not discussed here. It has been, however, reviewed and discussed in details by Weingart in Ref. ${ }^{193}$.

\subsection{Multiconfigurational and multireference methods}

Multiconfigurational self-consistent field (MCSCF), ${ }^{194}$ especially in the particular form of the complete active space self-consistent field (CASSCF), has been a frequent choice for NA-MQC simulations for different reasons, including its computational efficiency, the availability of analytical energy gradients and nonadiabatic couplings, its ability to describe regions in the PES with a significant multireference character, and availability in many computational chemistry packages. The main limitation of MCSCF is the lack of dynamical electron correlation, required to provide a balanced description of several regions in the PES. Moreover, the imbalance of between non-dynamical and dynamical electron correlations leads to dramatic overshoot of the ionic state energies. ${ }^{195-196}$ From the numerical point of view, the incompleteness of usual active spaces may render unstable dynamics, due to orbital rotations between subspaces, affecting the state description and energy conservation. ${ }^{197}$

Orbital rotations can be, in principle, controlled by enlarging the active space. The problem, naturally, is that the computational costs become quickly prohibitive. MCSCF, however, is flexible enough to deal with "by hand" built wavefunctions, where the active space is split into disjoint subspaces designed to address particular problems, at a minimum number of configurations. An example of such approach is in the TSH dynamics of ethylene reported in Ref. ${ }^{198}$. In that case, correlation beyond the minimal $\left(\pi, \pi^{*}\right)$ subspace was extended to the $(\sigma, \sigma *)_{\mathrm{CC}},(\sigma, \sigma *)_{\mathrm{CH}}$, and Rydberg orbitals in the MCSCF (and in the MRCI reference) by allowing full excitations within each subspace, and connecting the subspaces through single excitations.

From the methodological standpoint, the development and implementation of methods based on the density-matrix renormalization group SCF (DMRG-SCF) may boost MCSCF 
capabilities. ${ }^{199}$ These methods can deliver CAS-type wavefunctions with orbital spaces about five to six times larger than the CASSCF limits. Another promising emergent approach is the full configuration interaction quantum Monte Carlo method (FCIQMC), ${ }^{200-201}$ which may recover the full electron correlation at much lower computational scaling than conventional diagonalization of the full CI matrix.

Martínez's group has made significant efforts implementing ab-initio methods in GPU platforms. ${ }^{202-203} \mathrm{~A}$ new algorithm for CASSCF tailored for GPUs enables calculation of gradients and nonadiabatic couplings. ${ }^{204-205}$ These implementations have a positive scaling with respect to molecular size, extending the applications of NA-MQCs with CASSCF to systems with hundreds of atoms.

Some of the artifacts caused by the lack of dynamical electron correlation in CASSCF have been addressed from an empirical perspective by rescaling the potential energy surfaces to match fully correlated results. The initial efforts in this area, undertaken by Olivucci's group, ${ }^{206}$ have been recently updated by Martínez' group. ${ }^{207}$

Several of the MCSCF limitations may be overcome by post-treatment to recover dynamic electron correlation. Such methods may either be based on multireference configuration interaction (MRCI $)^{208-209}$ or multireference perturbation theory (MRPT). ${ }^{210-211}$ NA-MQC dynamics with MRCI, for which analytical gradients and analytical NACs are available,${ }^{212-214}$ has been reported. Nevertheless, the high cost of this method has restricted its application to strong CI truncation ${ }^{215}$ or small systems, as ethylene. ${ }^{198}$ The lack of implementation of analytical gradients and NACs for MRPT methods in general public codes has hampered their application in NAMQC. Martínez has led the use of complete active space perturbation theory to the second order (CASPT2) using MS. ${ }^{216-220}$ In a recent work, the González' group has performed TSH dynamics of a non-canonical nucleobase using MS-CASPT2 with numerically computed energy gradients and considering the effect of SOC. ${ }^{221}$

Park and Shiozaki ${ }^{222}$ have implemented an analytical gradient and NAC algorithm for CASPT2, which profits from a new factorization of the Lagrangian derivative terms with respect to the $\mathrm{CI}$ coefficients, to reduce the scaling of the calculations with the size of the active space. With that method, they have been able to deliver affordable computational costs for dynamics simulations at XMS-CASPT2 level. ${ }^{223}$ 
Semiempirical MRCI algorithms have also been used for NA-MQC dynamics. This is the case of the MRCI based on the orthogonalization method $x$ (OMx/MRCI, $x=1-3)$ from Thiel's group $^{224-226}$ and the floating occupation molecular orbital CI (FOMO-CI) from Granucci ${ }^{97,} 227$ based on AM1 and PM3 Hamiltonians. Strictly speaking, the FOMO-CI approach is not a multireference method, but its fractional occupation of virtual orbitals in the single reference determinant emulates a CASSCF wavefunction. ${ }^{27}$ The FOMO-CI approach has been implemented for an ab-initio Hamiltonian as well. ${ }^{228-229}$ Historically, the first on-the-fly TSH calculations ${ }^{56}$ were performed with the hybrid molecular mechanics / parameterized valence bond (MMVB) method developed by Bernardi, Olivuccci, and Robb ${ }^{230}$ to simulate CASSCF potential energy surfaces.

The most prominent advantage of such semiempirical approaches is the remarkably reduced computational costs, enabling an increase in the number of trajectories and reduction of time steps as compared to ab initio methods. However, the quality of such methods is intrinsically dependent on their parameterization ${ }^{231}$ and in many cases, parameterization is done for individual systems, ${ }^{109}$ not being directly transferable. Recent developments in machine learning algorithms applied to reparameterization have potential to boost these approaches. ${ }^{232}$ The absolute errors in OM2 atomization enthalpies computed for a benchmark of six thousand $\mathrm{C}_{7} \mathrm{H}_{10} \mathrm{O}_{2}$ constitutional isomers dropped from $6.3 \mathrm{kcal} / \mathrm{mol}$ with conventional parameterization, to astonishing 1.7 $\mathrm{kcal} / \mathrm{mol}$ with machine-learning parametrization.

Currently, most of the available multireference/multiconfigurational approaches based on density functional theory (DFT) ${ }^{233-239}$ do not count on analytical gradients, the minimum requirement for them to be coupled to NA-MQC methods. The exception is the recent implementation of analytical gradients for spin-restricted ensemble-referenced Kohn-Sham (REKS) method at (2 electrons, 2 orbitals) level. ${ }^{240}$ Non-dynamical electron correlation in DFT 241 — which Becke has claimed to be "the last frontier" 242 - is still an underdeveloped field despite recent advances in ensemble theory, ${ }^{240,} 243-244$ ensemble $^{245}$ and time-dependent ${ }^{236}$ hybrid multiconfigurational wavefunction and short-range DFT, and multiconfigurational DFT. ${ }^{246}$ 


\subsection{Single-reference methods}

\subsubsection{Nonadiabatic dynamics with single reference: does it make sense?}

In the early days of on-the-fly NA-MQC simulations, much of the attention was focused on the internal conversion from the excited state into the ground state when dealing with classical problems as ethylene ${ }^{6,97}$ or retinal $^{247}$ photodynamics. In such situation, the use of a multireference method is mandatory because, at the crossing seam where the conversion takes place, the ground state cannot be adequately described by a single reference. These initial studies may have led to the impression that NA-MQC dynamics must always be based on multireference methods. This is not strictly true. Many types of problem are restricted to the nonadiabatic evolution of the excited states only, where single-reference methods may perform well. ${ }^{248}$

Take for instance a fluorescent system. After the photoexcitation into a high electronic state, the molecule relaxes until reaching the minimum of the lowest excited state, where it remains oscillating up to it decays by photoemission. During the whole evolution of such a system, the ground state maintains a single reference character, and a single-reference method may be adequate for its description. In Fig. 7, we illustrate the molecular time evolution of the potential energy through a nonadiabatic process schematically. If the dynamics is somewhat restricted to regions like in $t_{0}, t_{1}$, or $t_{2}$, a single-reference method may work well, as the ground state wavefunction is dominated by a single determinant $|S R\rangle$. Note that even nonadiabatic couplings between excited states can be correctly described by single-reference methods. ${ }^{249}$

In fact, the emphasis on multiconfigurational/multireference methods to perform NA-MQC dynamics may have even led to some adverse effects. Many simulations have been based on CASSCF to describe the crossing seam with the ground state properly. Thus, to get right the nondynamical electron correlation affecting the dynamics during few tens of femtoseconds of motion near the crossing seam, these simulations completely neglected the dynamic electron correlation for hundreds of femtoseconds of the entire trajectory.

In addition to the observation that multireference description may not be an essential requirement, another feature favoring single-reference methods is that they are usually much faster than multireference calculations. These considerations have led to an increase in the popularity of single-reference methods in NA-MQC applications. 
Conversely, single-reference methods cannot be expected to be applicable to all kinds of problems. They will fail near an intersection with the ground state, as at time $t_{3}$ in Fig. 7. The topology of the intersection seam with the ground state will have wrong dimensionality. ${ }^{250-253}$ (This dimensionality problem is not restricted to single reference methods, and also affects single-state CASPT2 ${ }^{251}$ ) Many of the electronic-structure methods currently used for NA-MQC dynamics are based on linear response and, as such, they will not correctly describe double or multiple excitations, like the $\tau_{i j}^{a b}|S R\rangle$ state. In our hypothetical example (Fig. 7), this state is energetically above the energies of interest, but this may not always be the case. ${ }^{254}$

After converting to the ground state, the system may return to a single reference state, for instance, if it returns to the parent conformation. It may also continue through a multireference state, in the case of dissociation. Either way, NA-MQC simulations based on single-reference methods will not be adequate to describe this part of the dynamics ( $t_{4}$ in Fig. 7) because all events happening after the crossing the crossing to the ground state ( $\left.t_{3}\right)$ are not reliable. Our own strategy (and also of other groups ${ }^{253}$ ) in such cases has been to stop the trajectory propagation if the energy gap to the ground state drops below a certain threshold (usually $0.2-0.1 \mathrm{eV}$ ). This criterion must be considered with caution since the time needed for a particular system to decay through the crossing seam depends strongly on its nature. In our simulations for thymine based on algebraic diagrammatic construction to the second order $(\operatorname{ADC}(2))$, the effect of changing the energy gap threshold from 0.15 to $0.30 \mathrm{eV}$ reduced the $\mathrm{S}_{1}-\mathrm{S}_{0}$ time constant by $100 \mathrm{fs} .{ }^{255}$

Midway between multireference and single reference methods, the spin-flip (SF) strategy has become an alternative to include approximately multireference and double excitation effects at modest computational costs. In SF, calculations start from an unrestricted triplet ground state with $M_{S}=+1$. Excitations from this reference generate states with $M_{S}=0$, which may provide a reasonable description of conical intersections (as first suggested in Ref. ${ }^{250}$ ) and double excitations at the cost of spin contamination. SF has been developed and tested for DFT, CC, ${ }^{256} \mathrm{CI}$, ${ }^{257}$ and ADC ${ }^{258}$ methods. 


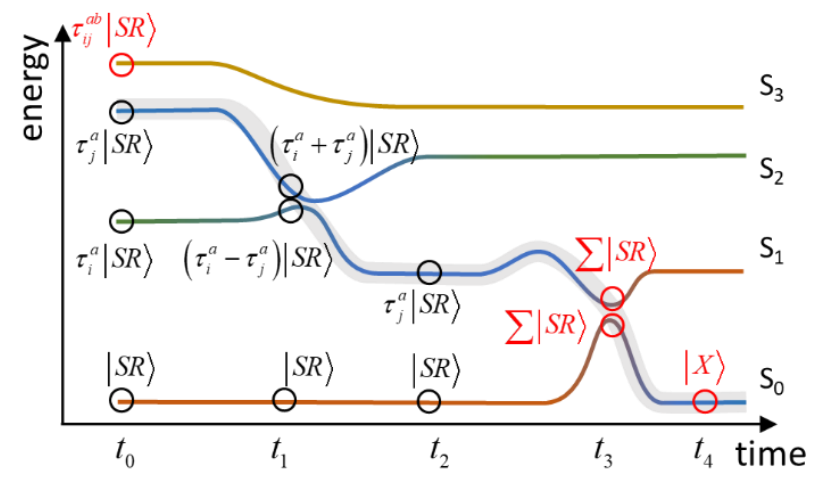

Fig. 7 Schematic time evolution of potential energy surfaces during the dynamics. Ground $\left(\mathrm{S}_{0}\right)$ and three excited states are indicated $\left(S_{1}\right.$ to $\left.S_{3}\right)$. The shaded curve shows the active state at each time. The main configuration dominating the wavefunction of each state at different time steps is given as well. $|S R\rangle$ indicates a single reference determinant. $\tau_{i}^{a}|S R\rangle$ indicates an electron promotion from orbital $i$ into orbital $a$ in the $|S R\rangle$ state. Analogously, $\tau_{i j}^{a b}|S R\rangle$ corresponds to a double excitation from $i, j$ into $a, b . \sum|S R\rangle$ means that more than one determinant is needed to describe the state.

The single-reference methods most commonly employed for on-the-fly NA-MQC dynamics may be grouped into two categories, real-time and linear-response methods, depending on how the electronic Schrödinger equation is addressed. They are reviewed in the next Subsections.

\subsubsection{Linear-response methods I: CC, $A D C$}

In response theory, the poles in the response function occur when the frequency of an external perturbation is equal to an eigenvalue of the stability matrix of the electronic structure method describing the unperturbed system. ${ }^{259}$ When the response function is expanded to contain up to linear terms in the perturbation (linear-response (LR) theory), the problem can be developed into a generalized eigenvalue equation

$$
\mathbf{A X}=\Omega \mathbf{X}
$$

where $\Omega$ is the excitation energy.

In coupled cluster (CC) theory, ${ }^{260-261}$ the matrix $\mathbf{A}$ is the Jacobian whose elements are

$$
\mathbf{A}_{\mu_{i} v_{j}}=\left\langle\Theta_{0}\left|t_{\mu_{i}}^{\dagger} \exp (-\hat{T})\left[\hat{H}_{e}, \hat{\tau}_{v_{j}}\right] \exp (\hat{T})\right| \Theta_{0}\right\rangle
$$


where $\hat{T}$ is the cluster operator formed by the product of the cluster amplitudes $t_{\mu_{i}}$ by the excitation operator $\hat{\tau}_{\mu_{i}} \cdot \mu_{i}$ represents an $i$-fold excitation, which is applied to the reference state, usually a Hartree-Fock wavefunction $\Theta_{0}$.

The CC Jacobian is a non-Hermitian matrix, which means that the excitations should be calculated twice, for the eigenvector acting at right (as in Eq. (62)) and also acting at the left:

$$
\begin{aligned}
& \mathbf{A R}=\Omega_{R} \mathbf{R}, \\
& \mathbf{L A}=\Omega_{L} \mathbf{L}, \\
& \mathbf{L}^{\dagger} \mathbf{R}=\mathbf{1}, \\
& \Omega_{R}=\Omega_{L} .
\end{aligned}
$$

As a consequence of $\mathbf{A}$ being non-Hermitian, when two excited states become degenerated, convergence problems in the determination of the excitations energies arise. ${ }^{262}$ Such lack of convergence renders LR-CC methods useless for NA-MQC dynamics. In Ref. ${ }^{263}$, TSH dynamics with LR-CC2 (coupled cluster to approximated second order) was tested and all trajectories failed within 100 fs due to numerical errors.

An alternative approach is to work with algebraic diagrammatic construction (ADC) for the polarization propagator. ${ }^{264-265}$ In this case, $\mathbf{A}$ (usually named M) is Hermitian, and the energy of degenerated excited states can be obtained by diagonalizing

$$
\begin{aligned}
& \mathbf{M X}=\Omega \mathbf{X}, \\
& \mathbf{X}^{\dagger} \mathbf{X}=\mathbf{1} .
\end{aligned}
$$

ADC is a general umbrella term for methods using the same approach for different propagators. ${ }^{266}$ Excitation energies are obtained from polarization propagators, but many other properties may be calculated as well, such as electron affinities from the electron propagator or ionization potentials from the hole propagator.

For practical implementations, ADC is derived in terms of basis of intermediate states, which are obtained by the action of excitation operators on a Møller-Pleset ground state. In general, if the correlated ground state is the MPn wavefunction, one arrives at the $\mathrm{ADC}(n)$ method. ${ }^{266} \mathrm{Up}$ to $\mathrm{ADC}(3)$, the $\mathbf{M}$ matrix is built in a space including single and double excitations. The most suitable level for NA-MQC in terms of accuracy/computational cost is ADC(2) (sometimes noted as $\mathrm{ADC}(2)$-s for strict), which provides single excitations at second-order order perturbation level. 
Nevertheless, in $\mathrm{ADC}(2)$, the couplings between doubles is zero, not describing adequately double excitations. An ad-hoc extension of ADC named ADC(2)-x adds these couplings to first order. ${ }^{267}$ The accuracy of this method is, however, low, and it is restricted to diagnosing the presence of doubles among the lowest excitations. ${ }^{268}$

A semiempirical parameterized version of ADC named SOS-ADC (for spin-opposite scaling) has also been implemented to reduce computational costs. It is derived by neglecting same-spin components in the doubles' contributions and rescaling (with the semiempirical factors) the opposite-spin contributions. In principle, SOS-ADC(2)-x could be an interesting semiempirical alternative for dealing with doubles in NA-MQC. The energetic separation between core and valence orbitals has been explored in ADC to separate these subspaces by neglecting their couplings in M. The CVS-ADC(2) (core-valence separated) can lead to accurate inner-shell excitation energies. ${ }^{269}$ The CVS-ADC could be the basis for NA-MQC-based simulations of innershell spectroscopy (see Section 5).

There is a link between ADC and $\mathrm{CC}$ theories that has been explored for efficient implementations of $\mathrm{ADC}$. $\mathrm{ADC}(2)$ matrix $\mathbf{M}^{A D C(2)}$ can be obtained as a symmetrized CC Jacobian. ${ }^{266}$ Starting from CCSD (coupled cluster with singles and doubles), if the double contributions are simplified to retain only the terms up to the lowest order in the fluctuation potential, it renders the CC2 approximation. ${ }^{260-261}$ Then, if in the CC2 Jacobian, the $t_{1}$ amplitudes are neglected, it leads to the $\operatorname{CIS}\left(\mathrm{D}_{\infty}\right)$ approximation, with Jacobian $\mathbf{A}^{\operatorname{CIS}\left(D_{\infty}\right)}$. Finally, the $\operatorname{ADC}(2)$ matrix can be written as $\mathbf{M}^{A D C(2)}=\left[\mathbf{A}^{C I S\left(D_{\infty}\right)}+\mathbf{A}^{C I S\left(D_{\infty}\right) \dagger}\right] / 2$.

Since the publication of the algorithm to evaluate approximate nonadiabatic couplings with $\mathrm{CC} 2$ and $\mathrm{ADC}(2),{ }^{263}$ different groups have used and implemented this methodology. ${ }^{255,270-280}$ So far, most of the ADC(2) NA-MQC dynamics simulations have focused on the photochemistry of heterocycles ${ }^{270-273}$ and the effect of aggregation with water on their excited-state dynamics..$^{274-278}$

A recent systematic study of some oligocenes and hetero-atomic fused rings has raised some concerns about the description of the $\mathrm{L}_{\mathrm{a}}$ and $\mathrm{L}_{b}$ states with both $\mathrm{CC} 2$ and $\mathrm{ADC}(2){ }^{281}$ Tuna et al. have shown that while $\mathrm{CC} 2$ is able to correctly describe the topography of the $\mathrm{S}_{1} / \mathrm{S}_{0}$ crossing in the protonated Schiff PSB3, a popular model for rhodopsin, ADC(2) (-s and $-\mathrm{x}$ ) produces intersection seams with the wrong dimensionality. ${ }^{252}$ In the case of CC methods, it has been 
recently shown that if the Jacobian matrix is nondefective, i.e., it can be diagonalized, the topology of the intersection seam is adequately described. ${ }^{282}$ In Ref. ${ }^{283}$, it is shown that the spin-flip ADC(3) level yields conical intersections with the correct dimensionality.

In line with these findings, it has been found that minimum energy conical intersections geometries obtained with $\mathrm{ADC}(2)$ can significantly deviate from the CASSCF geometries for some reaction coordinates. ${ }^{272,279}$ For instance, in the case of 2'-hydroxychalcone, where the deactivation to the ground state involves intramolecular rotation, the dihedral angle obtained with $\mathrm{ADC}(2)$ is $30^{\circ}$ smaller than that obtained with either CASSCF or CC2 ${ }^{279}$ This behavior, apparently associated with both the wrong description of the MP2 ground state and the ADC(2) excited state, has been explored by Szabla et al. considering NEVPT2 calculations. ${ }^{272}$ Constantly monitoring the dynamics with the $\mathrm{D}_{1}{ }^{284}$ and $\mathrm{D}_{2}{ }^{285}$ diagnostics may help to detect the build-up of multireference character in the ground state. (Note, however, that the $\mathrm{D}_{1}$ and $\mathrm{D}_{2}$ values recommended in Refs. ${ }^{284-}$ ${ }^{285}$ were derived for a too restricted set of molecules and are too small for many systems of interest. ${ }^{263}$ )

Another case to highlight is the photo-deactivation of pyrrole, where TD-B3LYP provides an appropriate description of the $\pi \pi^{*}$ states and time constants very similar to the experimental values. ${ }^{286}$ In contrast, $\operatorname{ADC}(2)$ shows an artificial mixing between the $\pi \pi^{*}$ and $3 p$ Rydberg and decay time constants more than three times longer than the experimental values.

Despite these problems, $\operatorname{ADC}(2)$ can still be considered a good option for NA-MQC dynamics, if its limits of validity are respected. The problems associated with the MP2 description of the ground state can be diagnosed with the $\mathrm{D}_{1}$ and $\mathrm{D}_{2}$ parameters, and by comparing with PES obtained with a correlated multiconfigurational method, such as CASPT2.

There is still a final problem affecting linear-response methods in general (also those discussed in the next section), which may impact dynamics: unphysical divergences. It occurs for a particular situation when the system has two states with excitation energies $\Delta E_{I}$ and $\Delta E_{J}$, and there is still a third state with excitation energy $\Delta E_{K}=\left|\Delta E_{J}-\Delta E_{I}\right|$. In such a case, spurious poles appear in the response function, leading to unphysical properties. This problem is discussed in detail in Ref. ${ }^{287}$. 


\subsubsection{Linear-response methods II: TDHF, TDDFT}

The derivation of the time-dependent Hartree-Fock (TDHF), also known as random phase approximation (RPA), starts from the electronic TDSE given in Eq. (6), but now adding an arbitrary single-particle time-dependent operator $\hat{v}_{e x t}(\mathbf{r}, t)$ to the electronic Hamiltonian $H_{e} \cdot{ }^{288-289}$ With the approximation that the time-dependent electronic wavefunction $\Psi(\mathbf{r}, t)$ can be described by a single determinant $\Theta(\mathbf{r}, t)$ of time-dependent molecular orbitals $\varphi_{i}^{H F}(t)$, the electronic TDSE is written as a time-dependent version of the Hartree-Fock equation ${ }^{290}$

$$
i \hbar \frac{\partial \varphi_{i}^{H F}}{\partial t}=\left[\hat{K}_{e}+\hat{v}_{c}+\hat{v}_{x}+\hat{v}_{e x t}\right] \varphi_{i}^{H F}
$$

where $\hat{K}_{e}$ is the electron kinetic energy operator, $\hat{v}_{c}$ and $\hat{v}_{x}$ are the electron-electron Coulomb and exchange interactions, and $\hat{v}_{\text {ext }}$ may contain additional arbitrary single-particle fields in addition to the electron-nuclei interaction.

The excited states for this model can once more be calculated from a linear-response (LR) approach, which in practical terms is reduced to the determination of the eigenvalues of the equation

$$
\left[\begin{array}{cc}
\mathbf{A} & \mathbf{B} \\
\mathbf{B}^{*} & \mathbf{A}^{*}
\end{array}\right]\left[\begin{array}{l}
\mathbf{X} \\
\mathbf{Y}
\end{array}\right]=\Omega\left[\begin{array}{cc}
1 & 0 \\
0 & -1
\end{array}\right]\left[\begin{array}{l}
\mathbf{X} \\
\mathbf{Y}
\end{array}\right],
$$

where the matrix elements are written in terms of orbital transitions from occupied orbitals $(i, j)$ into virtual orbitals $(a, b)$ as

$$
\begin{aligned}
& A_{i a, j b}=\delta_{i j} \delta_{a b}\left(\varepsilon_{a}-\varepsilon_{i}\right)+(i a \| j b), \\
& B_{i a, j b}=(i a \| b j) .
\end{aligned}
$$

In these equations, $(i a \| j b)$ is the anti-symmetrized two-electrons integral and $\varepsilon$ are orbital energies in the Mulliken notation. $\mathbf{B}=0$ defines the Tamm-Dancoff approximation (TDA), which in the TDHF case, it is merely the CIS approximation.

Mukamel and co-workers ${ }^{291-292}$ have explored TDHF and CIS combined with semiempirical Hamiltonians in a methodology named collective electron excitation (CEO), to 
efficiently compute excitation energies and optical response. The method has also been extended to provide energy gradients and nonadiabatic couplings. ${ }^{293}$ The CEO combined with FSSH and other TSH developments from Tretiak's group gives rise to the nonadiabatic excited-state molecular dynamics (NA-ESMD) method for NA-MQC dynamics. ${ }^{12,294}$

Kohn-Sham (KS) density functional theory (DFT) can also be recast in a time-dependent form analogous to TDHF. ${ }^{288-289,295-296}$ The main formal difference is that, in function of the RungeGross theorem relating the external potential and the density $\rho$, the TDSE is now written in terms of time-dependent Kohn-Sham orbitals $\varphi_{i}^{K S}(t)$ for the noninteracting system ${ }^{297}$

$$
i \hbar \frac{\partial \varphi_{i}^{K S}}{\partial t}=\left[\hat{K}_{e}+\hat{v}_{c}+\hat{v}_{x c}+\hat{v}_{e x t}\right] \varphi_{i}^{K S}
$$

where $\hat{v}_{x c}$ is the exchange-correlation potential. By construction, the density is related to the TDKS orbitals via

$$
\rho=\sum_{i}^{N}\left|\varphi_{i}^{K S}\right|^{2}
$$

In the linear response framework, the TDKS (or TDDFT) excitation energies are still given by the eigenvalues of Eq. (67), but with matrix elements

$$
\begin{aligned}
& A_{i a, j b}=\delta_{i j} \delta_{a b}\left(\varepsilon_{a}-\varepsilon_{i}\right)+(i a \mid j b)+\left(i a\left|f_{x c}\right| j b\right), \\
& B_{i a, j b}=(i a \mid b j)+\left(i a\left|f_{x c}\right| b j\right),
\end{aligned}
$$

where $f_{x c}$ is the exchange-correlation (xc) kernel and the integrals are still given in Mulliken notation. A fundamental approximation usually employed in TDDFT is the adiabatic local density approximation (ALDA), which assumes that the density varies slowly with time. ${ }^{297}$ This approximation allows using a local ground-state $f_{x c}$, delivering one of the most used methods for excited-state calculation.

In Eq. (71), the matrix elements are given for a pure density functional. They can be trivially extended to deal with hybrid functionals (see Eqs. (95) and (96) in Ref. ${ }^{288}$ ). Once more, $\mathbf{B}=0$ corresponds to TDA. ${ }^{298}$ 
Although LR-TDDFT has been exceptionally useful for NA-MQC dynamics ${ }^{299}$ it bears many limitations we should be aware of. An excellent discussion of them can be found in Ref. ${ }^{289}$, associated with specific approximations in the xc-functional $\left(E_{x c}\right)$, the xc-potential $\left(v_{x c}\right)$, and the xc-kernel $\left(f_{x c}\right)$. Particularly concerning NA-MQC dynamics, the most significant problems we may expect from conventional (ALDA) LR TDDFT are:

- Failure to describe bond breaking. During bond breaking, triplet and near-singlet instabilities arise. Moreover, an energetic switch between the HOMO and the LUMO along the dissociation causes numerical instabilities. The use of TDA and unrestricted approaches may alleviate these problems. ${ }^{253}$ In any case, it is better to avoid dissociative cases.

- Failure to describe conical intersections with the ground state. As a single reference method, Kohn-Sham DFT is not expected to adequately describe regions with multireference character in the ground state. Additionally, the substantial density variations at the crossing region challenge the validity of the adiabatic (ALDA) approximation. ${ }^{289}$ Finally, the crossing seam with conventional TDDFT has the wrong dimensionality, having one rather than two dimensions. ${ }^{250}$ There is not much to do to alleviate these problems but to avoid dynamics involving crossings to the ground state. The use of SF-TDDFT may be an option too. ${ }^{300-301}$ (An example of SF-TDDFT dynamics, but still without including nonadiabatic events, is discussed in Ref. ${ }^{302}$.)

- Failure to describe energies of charge-transfer states. Conventional TDDFT tends to underestimate the energy of states with small overlaps between the initial and final orbitals. ${ }^{303-304}$ This is a well-known problem related to the asymptotic behavior of $v_{x c}$ .$^{35,305-307}$ The standard solution has been to adopt range-separated functionals. ${ }^{308-309}$

- Failure to describe states with double and higher excitations. Due to the adiabatic approximation, $f_{x c}$ is limited to single excitations. ${ }^{295}$ The use of SF-TDDFT may once more be an option. ${ }^{300-301}$ The safe strategy is to monitor the excited states during the dynamics, with some auxiliary method able to diagnose the occasional presence of higher excitations in the spectral region of interest. One of such methods is the DFT/MRCI. ${ }^{233}$ 
- Failure to describe high-energy states. High-energy excited states tend to collapse between minus HOMO energy and the actual ionization potential. ${ }^{310}$ This renders an artificially large density of states in that region. ${ }^{311}$ There are some approaches to deal with this problem, ${ }^{310,312-313}$ but they have not been tested in NA-MQC dynamics.

An option to treat large systems still in the linear-response density functional level is to adopt the time-dependent density functional tight binding (TD-DFTB). ${ }^{314}$ In density functional tight binding, the ground-state reference density is written as a sum of the neutral densities of all atoms $^{315}$

$$
\rho_{0}=\sum_{\alpha} \rho_{0}^{\alpha} .
$$

This reference density is then perturbed by a fluctuation density $\delta \rho$ and the total energy is expanded in the respective orders: ${ }^{316}$

$$
E^{\text {DFTB }}[\rho]=\underbrace{\underbrace{E^{0}\left[\rho_{0}\right]+E^{1}\left[\rho_{0}, \delta \rho\right]}_{\text {DFTB1 }}+E^{2}\left[\rho_{0}, \delta \rho^{2}\right]}_{\text {DFTB3 }}+E^{3}\left[\rho_{0}, \delta \rho^{3}\right]+\cdots,
$$

giving rise to the diverse DFTB models. The commonly known self-consistent charge DFTB (SCC-DFTB) $)^{315}$ corresponds to the DFTB2 model. Thanks to a minimum basis set representation, the neglect of three-center integrals, and tabulated Hamiltonian and orbital overlap terms (which are calculated using atomic DFT), DFTB is an extremely efficient method. It is estimated, for instance, to be 1000 times faster than a B3LYP calculation. ${ }^{317}$

Electronic excitations for DFTB can be computed with the same LR-TD approach as employed for TDDFT (Eqs. (67) and (71)). ${ }^{314,318}$ The accuracy of TD-DFTB is limited by the accuracy of the functional used for its parameterization. ${ }^{319-320}$ In the same way, all problems we have discussed before occurring in DFT and TDDFT may be expected to occur in DFTB and TDDFTB.

LR TD-DFTB has been taken as the basis for NA-MQC dynamics with TSH by Mitric $e t$ $a l^{321}$ and more recently by Stojanović et al. ${ }^{322}$ DFTB has also been employed in other NA-MQC dynamics implementations but using real-time methods. They will be reviewed in Section 4.2.5. 
As a general recommendation when using LR-TDDFT, particular attention must be paid to the selection of the functional. This is especially important when the dynamics is determined by charge transfer states, such as in excited-state proton transfer ${ }^{323}$ and organic photovoltaics applications. $^{324}$

Applications of TSH using LR-TDA/TDDFT excited states include studies of the deactivation of organic molecules with biological applications, ${ }^{325-329}$ excited state proton transfer, ${ }^{330-331}$ atmospheric chemistry, ${ }^{332}$ and organometallic complexes. ${ }^{333-335}$ A recent study from Muuronen et al. has explored the dynamics of water absorbed on $\mathrm{TiO}_{2}$ clusters. ${ }^{336}$ Exciting applications with impact in organic photovoltaics include the study of hot charge-transfer states in bithiophene dimer and the investigation of the ultrafast energy transfer in an orthogonal molecular dyad. $^{324,337}$

\subsubsection{Real-time methods I: frozen nuclei}

The solution of either the TDHF (Eq. (66)) or the TDKS equations (Eq. (69)) can be directly obtained by integration in the real-time (RT) domain. ${ }^{290,338}$ (We highlight that at this level, the goal is to simulate the electron dynamics with frozen nuclei.) The integration of the EOMs may be based on several frameworks, ${ }^{339}$ including real-space grids, ${ }^{340}$ plane waves, ${ }^{341}$ and localized atomic orbitals. ${ }^{342}$ In the RT formalism, the excited states are not explicitly computed but described as a coherent superposition in the Ehrenfest approximation, ${ }^{343}$ the MFE approach discussed in Section 2.1. The information on the excited states appears as population transfer between orbitals, induced by the external field, usually an oscillating electric field. After Fourier transforming the dipole moment evolution with time, the excitation spectrum is obtained. ${ }^{344}$

The computation of the spectrum in the RT formalism requires thousands of time steps. For example, the spectrum reported in Ref. ${ }^{345}$ was computed by propagating the TDKS by 25 fs with 0.002 fs time step (i.e., 12,500 time-steps), with frozen nuclei. Even though the integrals to form the Fock matrix do not need to be computed at every time step $^{290}$ and the current implementations count on massive parallelization, ${ }^{346}$ the computational costs associated to getting excited states via real-time approaches are still overwhelming if compared to that those at linearresponse theory. 
Beyond computational efficiency, other factors are favoring LR over RT approaches. For approximated wavefunctions (and this is always the case), the expected values of an observable obtained via linear response tend to be more accurate than when obtained via real-time integration; moreover, LR approaches allow getting the properties of many states at once. ${ }^{287}$ Together, all these reasons have contributed to the dominance of LR methods in the current quantum chemistry of excited states.

\subsubsection{Real-time methods II: electron-nucleus coupling}

The RT approach is a direct entry door to NA-MQC dynamics within the Ehrenfest formalism, by coupling the electronic response to the nuclear motion. At this point, nonadiabatic dynamics and electronic structure calculations merge into the same methods. ${ }^{347}$ The external single-particle potential $\hat{v}_{\text {ext }}$ appearing in Eqs. (66) and (69) is the key for this coupling, as it contains the electron-nuclei interaction $\hat{v}_{e-n}$. For the simple determination of electronic excitations, RT approaches will take the nuclei frozen, and $\hat{v}_{e-n}$ is constant. (The time dependence of $\hat{v}_{\text {ext }}$ is restricted to the applied field.) For NA-MQC dynamics, the nuclear motion along trajectories is considered, implying that $\hat{v}_{e-n}(t)$ is now a function of time as well.

At this level, the time evolution of the molecular system gains an elegant description within the time-dependent self-consistent approach: ${ }^{35}$ not only the electrons respond to the timedependent field of the nuclei through $\hat{v}_{e-n}(t)$, but the new time-dependent electronic state determines the forces acting on the nuclei through the mean field force in Eq. (12).

In practical terms, the main difficulty in MFE TDHF/TDKS dynamics is to deal with the significant difference between the time-step needed to integrate the electronic (TDSE) and nuclear (classical) equations, which is of the order of 10 to 100 times larger in the latter. $\mathrm{Li}$ and coworkers $^{343}$ have proposed a three time-step integrator to address this problem. In their algorithm, implemented for TDHF, the classical equations are integrated with the largest time step. The electronic integrals to form the Fock matrix are computed for intermediary time-steps, and the TDHF equations are integrated with the smallest time steps. 
As in the case of fixed nuclei, MFE dynamics based on RT-TDKS has several implementations based on real-space grids, ${ }^{340}$ plane waves, ${ }^{341}$ and atomic orbitals. ${ }^{342}$ It also counts on DFTB extensions. ${ }^{348}$ In a basic implementation working on atomic orbitals $\vartheta_{p}$, the TDKS orbitals are given as ${ }^{342}$

$$
\begin{aligned}
& \varphi_{i}(t)=\sum_{p} c_{i p}(t) \vartheta_{p}(\mathbf{r} ; \overline{\mathbf{R}}), \\
& S_{p q}=\left\langle\vartheta_{p} \mid \vartheta_{q}\right\rangle,
\end{aligned}
$$

which leads to the equation-of-motion

$$
\frac{d \mathbf{c}_{i}}{d t}=-\mathbf{S}^{-1}\left[\frac{i}{\hbar} \mathbf{H}^{K S}+\boldsymbol{\sigma}^{N A C}\right] \mathbf{c}_{i}
$$

In this equation, $H_{p q}^{K S}=\left\langle\vartheta_{p}\left|H^{K S}\right| \vartheta_{q}\right\rangle$ are the matrix elements of the KS-Hamiltonian (the terms in the brackets of the right side of Eq. (69)) and $\sigma_{p q}^{N A C}=\left\langle\vartheta_{p} \mid \dot{\vartheta}_{q}\right\rangle$.

Conceptually, extending the RT-TDHF or RT-TDKS approaches to TSH does not follow as readily as it does for MFE. In the exact (fully coherent) method, the wavefunction propagated by the TDSE (Eq. (6)) is written as a linear combination of many-electron states (Eq. (7)). TSH follows naturally by imposing that the propagation should take place on just one of these manyelectron states. In TDHF and TDKS, however, the TDSE (Eqs. (66) and (69)) propagate singleelectron wavefunctions (orbitals), and there is no information about on which many-electron state the trajectory should be propagated.

Despite this conceptual difficulty, RT-TDKS has been adapted to TSH, and it has been intensively explored by Prezhdo's group in the last decade. ${ }^{349-351}$ In their method, the external potential acting on the noninteracting system is also taken to be the time-dependent electrostatic field of the nuclei, while the TDKS orbitals $\varphi_{i}(\mathbf{r}, t)$ are expanded on the basis of time-independent KS orbitals $\tilde{\varphi}_{i}(\mathbf{r} ; \overline{\mathbf{R}})$

$$
\varphi_{i}(\mathbf{r}, t)=\sum_{j} c_{i j}(t) \tilde{\varphi}_{j}(\mathbf{r})
$$

This expansion leads to the equation-of-motion 


$$
\frac{d c_{i j}}{d t}=-\sum_{k}\left[\frac{i}{\hbar} \varepsilon_{k} \delta_{j k}+\sigma_{j k}^{N A C}\right] c_{p k}
$$

where $\varepsilon_{k}$ are the KS orbital energies and $\sigma_{j k}^{N A C}=\left\langle\varphi_{j} \mid \dot{\varphi}_{k}\right\rangle$. A many-electron time-dependent coefficient is formed by the product of the single-electron coefficients c. They are then used to calculate the populations and coherences needed to obtain the FSSH probability (Eq. (15)).

Effectively, the TDKS information is used only to compute FSSH probabilities, as the nuclei are propagated on excited states given as single determinants composed of time-independent KS orbitals. ${ }^{349}$ (We will refer to this method as time-dependent single-determinant Kohn-Sham, TD-SDKS.) In this way, the excitation energies are reduced to bare KS gaps, i.e. the difference between KS orbital energies. ${ }^{351}$ Such approximation leads to enormous computational savings, allowing to treat extensive systems, ${ }^{166,352-353}$ well beyond the limits of LR-TDDFT. NA-MQC dynamics of systems composed of thousands of atoms are becoming a real possibility for the extension of this methodology to DFTB. ${ }^{354-355}$

The price to pay for such computational efficiency of the TD-SDKS is in the low accuracy of the electronic states. Maitra ${ }^{356}$ pointed out that the single-determinant Kohn-Sham approximation does not form an adequate basis for describing adiabatic states, with potential energy curves resembling more those of diabatic states. Compared to LR-TDDFT, this poor description of the energies arises due to two factors: first, the neglect of the contributions of the Hartree and exchange-correlation kernels (the two last terms in the right side of the matrix elements $A_{i a, j b}$ in Eq. (71)); second, the lack of mix with other singly-excited configurations resulting from the diagonalization of Eq. (67) ${ }^{356}$ (see also the discussion in Ref. ${ }^{28}$ ).

Fischer et al. $^{350}$ have presented benchmark results for TSH based on TD-SDKS and LRTDDFT for few different systems, showing a reasonable agreement between them. Stojanović et al. also compared SDKS results (based on DFTB) to TD-DFTB. ${ }^{322}$ A reasonable agreement between these methods was found too. Nevertheless, it tends to degrade for delocalized densities. Although these benchmarks help to understand the accuracy of TD-SDKS beyond the conceptual discussion, we believe that more extensive comparisons are still needed to truly settle the matter.

The description of excited states in terms of a single KS determinant in the TD-SDKS ${ }^{349}$ invokes the NA-MQC dynamics based on the restricted open-shell Kohn-Sham (ROKS) approach, 
which was one of the first available methods for on-the-fly surface hopping, proposed by Doltsinis and Marx. ${ }^{357-358}$ The main difference is that in ROKS the excited state (limited to $\mathrm{S}_{1}$ ) was given by a spin-adapted sum of two KS determinants, whose KS orbitals were independently determined for the $\mathrm{S}_{0}$ and $\mathrm{S}_{1}$ states. Additionally, in the Doltsinis-Marx approach, the nuclei were propagated via Car-Parrinello molecular dynamics (CPMD), ${ }^{359}$ rather than the usual Bohr-Oppenheimer molecular dynamics (BOMD).

\subsection{Calculation of couplings}

One of the keys parameters to perform NA-MQC dynamics is the nonadiabatic coupling. Analytical NACs are available for multiconfigurational approaches such as MCSCF and MRCI. ${ }^{213-}$ ${ }^{214}$ Analytical NACs for MS-CASPT2 were derived and applied for AIMS calculations. ${ }^{216-217}$ They have also been implemented for XMS-CASPT2 222 and employed for QM/MM TSH dynamics. ${ }^{223}$ As already mentioned (Section 4.1), the method presented in Ref. ${ }^{222}$ shows a reduced scaling of the calculations with the size of the active space.

Different formulations for analytical NACs are available for (LR) TDDFT. ${ }^{360-363}$ The theoretical foundation for calculating these couplings has been established by Baer $^{364}$ and Chernyak and Mukamel. ${ }^{360} \mathrm{Hu}$ and co-workers have further developed the Chernyak-Mukamel approach to calculating nonadiabatic coupling vectors between the ground state and the first excited state. ${ }^{365-367}$ Tavernelli and co-authors ${ }^{362}$ derived an expression for NAC between any pair of states based on Casida's auxiliary multielectron wavefunctions (AMEW). ${ }^{295}$ Send and Furche ${ }^{361}$ showed that Hu's implementations neglected molecular orbitals derivatives, and proposed their own derivation including such terms. Tavernelli's derivation has also been criticized ${ }^{361}$ on the basis of imposing too strong approximations when treating the derivative coupling as a one-electron operator. Fully Chernyak-Mukamel complying derivations of NAC have been provided by Send and Furche ${ }^{361}$ for ground/excited states and by Ou et al. ${ }^{368}$ for excited/excited states.

The equations of motion for MFE and TSH (see Eq. (8)) can be evaluated without explicitly calculating the NACs $\left(\mathbf{d}_{J K}\right)$ by using the time-derivative couplings $\sigma_{J K}^{N A C}$. This is particularly useful in the case of methods and programs for which analytical NACs are not available. The $\sigma_{J K}^{N A C}$ couplings can be evaluated using finite differences as proposed by Hammes-Schiffer and Tully (HST) as ${ }^{42}$ 


$$
\sigma_{J K}^{N A C}\left(t+\frac{\Delta t}{2}\right) \approx \frac{1}{2 \Delta t}\left[S_{J K}(t+\Delta t)-S_{K J}(t+\Delta t)\right]
$$

where

$$
S_{J K}(t)=\left\langle\psi_{J}(t-\Delta t) \mid \psi_{K}(t)\right\rangle
$$

are wavefunction overlaps between consecutive time steps. This expression can be more conveniently written as ${ }^{369}$

$$
\sigma_{J K}^{N A C}(t) \approx \frac{1}{4 \Delta t}\left[3 S_{J K}(t)-3 S_{K J}(t)-S_{J K}(t-\Delta t)+S_{K J}(t-\Delta t)\right]
$$

The approximate couplings obtained within the HST scheme are in good agreement with the analytical couplings. 370

For methods with explicit wavefunctions (MCSCF, MRCI, etc.), Eq. (80) can be directly used. ${ }^{370}$ In the case of linear-response methods (CC, ADC, TDDFT, etc.), an AMEW corresponding to the configuration interaction Ansatz

$$
\left|\psi_{K}\right\rangle=\sum_{i a} C_{i a}^{K}\left|\Theta_{i}^{a}\right\rangle
$$

is considered, where $\left|\Theta_{i}^{a}\right\rangle$ are Slater determinants (with a single excitation from orbital $i$ into orbital $a$ ) and the linear-response coefficients $X_{i a}^{K}$ from Eq. (62) are taken for $C_{i a}^{K}: 263,371-373$

$$
C_{i a}^{K}=\left\{\begin{array}{ccc}
R_{i a}^{K}, L_{i a}^{K} & \text { for } & C C, \\
M_{i a}^{K} & \text { for } & A D C, \\
(X+Y)_{i a}^{K} & \text { for } & T D D F T .
\end{array}\right.
$$

Meek and Levine have proposed that a better approximation than the HST may be obtained if the couplings are averaged over the time interval after interpolating the wavefunctions using a unitary transformation. ${ }^{100}$ In their norm-preserving interpolation (NPI) scheme, the couplings are

$$
\sigma_{J K}^{N A C}=\frac{1}{\Delta t} \int_{t-\Delta t}^{t} d \tau\left\langle\psi_{J}(\tau) \mid \frac{\partial \psi_{K}(\tau)}{\partial \tau}\right\rangle,
$$

where $|\psi(\tau)\rangle=\mathbf{U}(\tau)|\psi(t-\Delta t)\rangle$ and the transformation matrix is given as 


$$
\begin{aligned}
& U_{J J}(\tau)=\cos \left(\frac{\tau-(t-\Delta t)}{\Delta t} \cos ^{-1}\left(S_{J J}(t)\right)\right), \\
& U_{J K}(\tau)=\sin \left(\frac{\tau-(t-\Delta t)}{\Delta t} \sin ^{-1}\left(S_{J K}(t)\right)\right) .
\end{aligned}
$$

The multistate generalization of this formalism is derived in Ref. ${ }^{71}$, where it is discussed that the NPI approach effectively takes the logarithm of the overlap matrix, allowing for considerable time scale speed-up.

With the implementation of NA-MQC dynamics based on semiempirical methods, the computation cost associated with the coupling evaluation becomes a serious issue. Some savings may be made if the number of states to be considered in the calculation of the couplings is reduced. ${ }^{337,370,374}$ Moreover, the couplings may be estimated from the CI coefficient alone, neglecting the orbital derivatives, as done in Refs. ${ }^{375-378}$

Usual implementations of the HST approach calculate time derivatives (and wavefunction overlaps) on the basis of Slater determinants. ${ }^{370,379}$ Such determinant derivative (DD) approaches approximately scale to $N_{o c c}^{5} N_{v i r t}^{2}$ for a CIS-type wavefunction, where $N_{o c c}$ and $N_{v i r t}$ are the numbers of occupied and virtual orbitals. This scaling results from the number of determinant pairs $\left(\left(N_{o c c} N_{\text {virt }}\right)^{2}\right)$ times the cost of computing the overlap between two nonorthogonal determinants, which using the Lödwin formula ${ }^{380}$ scales to $N_{o c c}^{3}$. The computational effort can be largely reduced if certain determinant overlaps terms are neglected when the product of their CI coefficients drops below some threshold or if the determinant's excitation rank is too high. ${ }^{370}$ Plasser et al. ${ }^{381}$ have shown that there are substantial redundancies in the overlap calculations. If repeated terms are stored and reused, the effective scaling in their optimized-DD approach reduces from $N_{o c c}^{5} N_{v i r t}^{2}$ to $N_{o c c}^{4} N_{v i r t}$. (See also Refs. ${ }^{382-383}$ for some historical background on such optimized wavefunctions.)

A different approach to compute the time derivative couplings within the HST approach been proposed by Ryabinkin, Nagesh, and Izmaylov. They have shown that if the time derivatives of a CIS-type wavefunction are computed on the basis of molecular orbitals (orbital derivative approach, OD), rather than in a basis of determinants, the scaling is then reduced from $N_{o c c}^{5} N_{v i r t}^{2}$ in the full-DD approach to $N_{o c c} N_{v i r t}^{2} \cdot 384$ 
Both the optimized-DD and OD approaches have shown excellent results in comparison to the full DD, with significantly reduced computational costs involved in the evaluation of the couplings. The optimized-DD approach, however, counts with a couple of the advantages over the OD approach: it scales better to $N_{\text {virt }}$ and it can be directly applied to high excitation ranks. The OD algorithm has been recently implemented for TSH, being available for TDDFT, TD-DFTB, CIS, ADC(2), and CC2. ${ }^{255}$ The optimized-DD algorithm has also been implemented for TSH, and it is available for MCSCF, MRCI, ${ }^{381}$ and TDDFT. ${ }^{385}$

The use of AMEW based on LR coefficients as those in Eqs. (81) and (82) has been generalized $^{263,299}$ and popularized beyond the computation of time-derivative NACs. These auxiliary functions have been used for the calculation of various other properties including spinorbit couplings, ${ }^{170,373,386}$ transition dipole moments, ${ }^{362,387}$ nonadiabatic coupling vectors, ${ }^{362,} 388$ and Dyson orbitals. ${ }^{389-390}$

\section{Spectroscopic simulations based on NA-MQC dynamics}

During the execution of NA-MQC dynamics, the semi-classical nuclear phase space is populated, generating an ensemble of nuclear geometries and momenta in different electronic states, and distributed as a function of time. The nuclear ensemble approach (NEA) can be explored to simulate different steady and time-resolved spectroscopic techniques, including inhomogeneous broadening. ${ }^{391-392}$

Usually, working as a post-processing of the dynamics results, the nuclear ensembles have been applied for simulations of a large variety of time-resolved spectra, including twodimensional, differential transmission, ${ }^{393}$ photoelectron, ${ }^{24,217,325,389-390,394-397}$ ultrafast Auger, ${ }^{24,398}$ and X-ray photo-scattering ${ }^{24}$ spectroscopies. These developments and applications have been based on a broad range of approximations and electronic structure methods, from simple estimates of transition probabilities ${ }^{24,325,387,395,399-401}$ to involved modeling of transition moments. ${ }^{217,389,396,}$ 400, 402-403 


\subsection{Steady-state spectroscopy}

\subsubsection{Photoabsorption spectrum}

Photoabsorption spectra are usually simulated before NA-MQC dynamics as a way to generate initial conditions for the trajectories. In a typical situation, an ensemble $\eta(\mathbf{R}, \mathbf{P})$, containing $N_{p}$ phase-space points with nuclear coordinates and momenta $\mathbf{R}$ and $\mathbf{P}$, is created in the ground state by either propagating long ground-state trajectories or sampling some adequate phasespace distribution. A convenient option is to assume that the ground state motion is harmonic and employ a Wigner distribution for the quantum harmonic oscillator, to sample normal coordinates ${ }^{92,}$ 404-405

$$
P^{W}(\mathbf{q}, \mathbf{p})=\frac{1}{(\pi \hbar)^{3 N_{a t}-6}} \prod_{i=1}^{3 N_{a t}-6} \exp \left(\frac{-q_{i}^{2}}{2 \varpi_{q i}^{2}}\right) \exp \left(\frac{-p_{i}^{2}}{2 \varpi_{p i}^{2}}\right)
$$

where

$$
\begin{aligned}
& \varpi_{q i}^{2}=\frac{\hbar}{2 \mu_{i} \omega_{i}}, \\
& \varpi_{p i}^{2}=\frac{\hbar \mu_{i} \omega_{i}}{2} .
\end{aligned}
$$

In these equations, $q_{i}$ and $p_{i}$ are the coordinates and momenta for each normal mode $i$ with reduced mass $\mu_{i}$ and angular frequency $\omega_{i}$. (The impact of choosing either trajectories or distributions to build the ensemble is discussed in Section 7.) Whatever method is chosen to build the ensemble $\eta(\mathbf{R}, \mathbf{P})$, as soon as it is ready, the photoabsorption cross section can be computed as ${ }^{406}$

$$
\sigma^{p a}(E)=\frac{\pi e^{2} \hbar}{2 m_{e} c \varepsilon_{0} E} \sum_{L}^{N_{f s}} \frac{1}{N_{p}} \sum_{n}^{N_{p}} \Delta E_{1 L}\left(\mathbf{R}_{n}\right) f_{1 L}\left(\mathbf{R}_{n}\right) w_{s}\left(E-\Delta E_{1 L}\left(\mathbf{R}_{n}\right), \delta\right)
$$

where $E$ is the photon energy, $\varepsilon_{0}$ is the vacuum permittivity, $c$ is the speed of light, and $e$ and $m_{e}$ are the electron charge and mass. $w_{s}$ is a normalized sharp line shape (Gaussian or Lorentzian, for instance) centered at the vertical transition energy $\Delta E_{1 L}$ between the ground state (state 1) and the electronic state $L(L>1)$, computed at for each of the ensemble geometries $\mathbf{R}_{n} . f_{1 L}$ is the oscillator strength between the two states at the same geometry. A total of $N_{f s}$ electronic states are included. 
The parameter $\delta$ is the width of the line shape function $w_{s}$. Because this parameter is usually chosen to be much narrower than the width of the absorption band, it does not significantly interfere with the simulated spectrum.

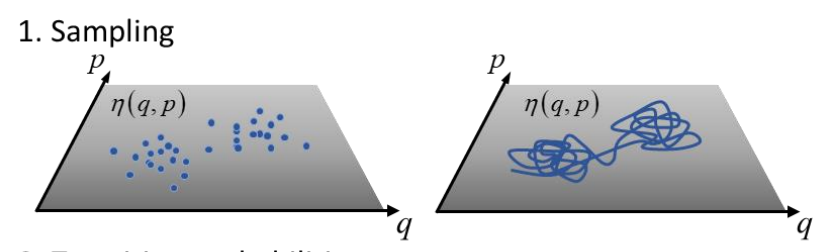

2. Transition probabilities

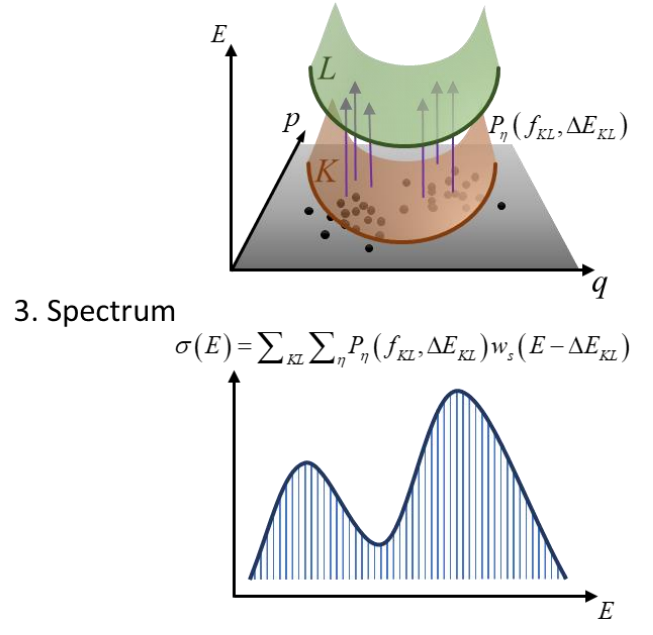

Fig. 8 Spectrum simulation with the nuclear ensemble approach. 1. The nuclear phase space in the source state $K$ is populated either with a probability distribution function (left) or via dynamics (right). 2. For each point in the ensemble, the transition probability between the source state $K$ and the target state $L$ is computed. This probability is a function of the transition moment (represented by the oscillator strength $f$ in the figure) and of the resonant energy $\Delta E_{K L}$ between the states. 3 . The spectrum is obtained as an incoherent sum of these transition probabilities, broadened by a thin line shape function. The spectrum can be used to select initial conditions for NA-MQC dynamics as well as be the result of post-processing NA-MQC simulations. Several types of spectrum (absorption, emission, photoelectron, time-resolved, etc.) can be simulated by appropriate choice of the initial ensemble definition, the states involved, and the probability function.

The absorption spectrum computed with the NEA (see Fig. 8), Eq. (87), is able to provide a reasonable approximation for the absolute width and absolute height of the absorption bands. It also allows estimating the shift between the vertical excitation and the band maximum and, because it goes beyond the Condon approximation, it delivers the intensity of dark vibronicallycoupled bands as well. ${ }^{406}$ The NEA, however, does not contain any information about the vibrational structure and it does not recover the band asymmetry well. ${ }^{407}$ 
The shortcomings of the NEA are related to the lack of information on the excited states (beyond the vertical excitation energies). We may recall that from a time-dependent perspective, the photoabsorption is given by the Fourier transform of the dipole correlation function, ${ }^{408}$ i.e.

$$
\sigma^{p a}(\omega) \propto \omega \operatorname{Re}\left[\int_{0}^{\infty} d t e^{i \omega t} C_{\mu \mu}(t)\right],
$$

where $\omega=E / \hbar$ and

$$
C_{\mu \mu}(t)=\langle\Phi(0)|\hat{\mu}(t) \hat{\mu}(0)| \Phi(0)\rangle
$$

In this equation, $\Phi(t=0)$ is the initial molecular wavefunction (see Eq. (3)) and $\hat{\mu}$ the dipole operator.

In Ref. ${ }^{406}$, we discuss which approximations should be imposed to $C_{\mu \mu}$ so that the photoabsorption cross section is reduced to Eq. (87). In fact, it is shown that the sum over ensemble points $n$ in Eq. (87) corresponds to a Monte Carlo integration over the nuclear coordinates implicit in Eq. (89)

$$
\int d \mathbf{R} \eta(\mathbf{R}) f(\mathbf{R}) \rightarrow \frac{1}{N_{p}} \sum_{n \in \eta}^{N_{p}} f\left(\mathbf{R}_{n}\right) .
$$

In the late 1960s, $\mathrm{Kubo}^{409}$ laid the grounds to compute $C_{\mu \mu}$ from an ensemble of trajectories. Ben-Nun and Martínez also directly evaluated Eq. (89) from MS wavefunctions to calculate absorption and Raman spectra. ${ }^{410}$ More recently, Petit and Subotnik proposed a hierarchy of approximations involving trajectories propagation in the ground and excited states, to obtain $C_{\mu \mu} \cdot{ }^{411-412}$ Moreover, they have derived expressions for both, MFE and TSH, showing that $C_{\mu \mu}$ can be written as a function of the elements of the density matrix $\boldsymbol{\rho}^{(n)}=\mathbf{c}^{(n) *} \mathbf{c}^{(n)}$ coming from Eq. (8) for each trajectory $n$. Such approach goes much beyond the NEA and allows to recover the full band shape, including the vibrational structure.

All methods discussed above are related to the time-dependent approach to the spectrum, as given by Eq. (88). TSH has also been used to provide complementary information to the conventional energy-eigenvalue approach, where the spectrum is evaluated as a sum over all 
vibronic transitions between states vibrational level $m$ of the electronic ground state and the vibrational level $n$ of the electronic state $L:^{413}$

$$
\sigma^{p a}(E) \propto E \sum_{1 m} \eta_{0 m}^{B o l t z} \sum_{L n}\left|\mu_{1 m, L n}\right|^{2} \delta\left(E-\Delta E_{1 m, L n}\right)
$$

In this equation, $\eta_{0 m}^{\text {Boltz }}$ is a Boltzmann distribution of initial states and $\mu_{1 m, L n}$ are transition dipoles moments. Such an approach results in a stick spectrum, which is usually broadened by shape functions with arbitrary width. Using the fact that the shape function width is related to the excitedstate lifetime, Röhr, Mitrić, and Petersen ${ }^{414}$ have proposed to use lifetimes fitted from a short surface hopping dynamics to provide a parameter-free spectral simulation.

\subsubsection{Photoemission spectrum}

The steady-state emission spectrum is analogous to photoabsorption, with the difference that the ensemble should be built for the excited states. Assuming the validity of the Kasha's rule, the differential rate of expontaneous radiative emission (dimensionless) is given by ${ }^{406}$

$$
\Gamma^{\mathrm{rad}}(E)=\frac{e^{2}}{2 \pi \hbar m_{e} c^{3} \varepsilon_{0}} \frac{1}{N_{p}} \sum_{n}^{N_{p}} \Delta E_{21}\left(\mathbf{R}_{n}\right)^{2} f_{12}\left(\mathbf{R}_{n}\right) w_{s}\left(E-\Delta E_{21}\left(\mathbf{R}_{n}\right), \delta\right) .
$$

In the case of stimulated emission, the spectrum may still be computed with Eq. (87). For steady phosphorescence, Eq. (92) can be used, but with oscillator strengths and energy transitions calculated in a spin-mixed representation. This result, obtained in the nuclear ensemble approach, shares the same advantages and disadvantages as those discussed in the previous Section for the photoabsorption.

The ensemble used to compute the emission spectrum may still be obtained using the distribution function in Eq. (85), but, adapted to the excited-state normal modes. Alternatively, the ensemble can be built from NA-MQC trajectories. ${ }^{415}$ For a steady-state spectrum simulation, the initial transient dynamics should be discarded, and only points obtained after equilibration in the minimum of the excited states should be considered. 


\subsubsection{Photoelectron spectrum}

The steady photoelectron emission intensity in the NEA is (area/energy) ${ }^{390}$

$$
\Gamma\left(E_{b}\right)=\sum_{\kappa} \frac{1}{N_{p}} \sum_{l=1}^{N_{p}} \sigma_{I \kappa}\left(E_{b}, \mathbf{R}_{n}\right) w_{s}\left[E_{b}-\Delta V_{I \kappa}\left(\mathbf{R}_{n}\right), \delta\right],
$$

where, the binding energy $E_{b}=E-K_{e}$ is defined in terms of the photon energy $E$ and the kinetic energy of the emitted electron $K_{e} \cdot w_{s}$ is a normalized sharp line shape with a width $\delta$, centered at the ionization potential $\Delta V_{I \kappa}$ calculated between the initial state $I$ of the $N$-electron molecule and the state $\kappa$ of the $(N-1)$-electron molecule. $\sigma_{I \kappa}$ is the ionization cross section between $I$ and $\kappa$. Both $\sigma_{I \kappa}$ and $\Delta V_{I \kappa}$ are computed for each nuclear geometry $\mathbf{R}_{n}$ in the ensemble.

The ionization cross section is given as ${ }^{416-417}$

$$
\sigma_{I \kappa}=\frac{\pi}{3} \frac{m_{e} g_{I}}{\varepsilon_{0} c \hbar^{3}} k E\left|\left\langle F_{k}|\mu| \phi_{I \kappa}^{D}\right\rangle_{\mathbf{r}_{N}}\right|^{2}
$$

where $k$ is the momentum of the ejected electron, whose wavefunction is $\left|F_{k}\right\rangle \cdot\left|\varphi_{I \kappa}^{D}\right\rangle$ is the Dyson orbital

$$
\varphi_{I \kappa}^{D}\left(\mathbf{r}_{N}\right)=\sqrt{N}\left\langle\psi_{\kappa}^{N-1} \mid \psi_{I}^{N}\right\rangle_{\left\{\mathbf{r}_{N-1}\right\}}
$$

Alternatively, the photoionization cross section can be simply approximated for

$$
\sigma_{I \kappa} \propto\left\|\varphi_{I \kappa}^{D}\right\|^{2}
$$

For a more rigorous treatment of the continuum states, see Ref. ${ }^{418}$.

\subsection{Time-resolved spectroscopy}

\subsubsection{Pump-probe spectrum}

Time-resolved pump-probe spectroscopy has been fundamental to reveal the nonadiabatic dynamical evolution of molecular systems on the femtosecond scale. ${ }^{419-420}$ Typical experimental setups employ two laser pulses hitting the molecule with a controllable delay. The first pulse excites the system (UV/vis for pumping valence states; X-Ray for core states), while the second 
pulse probes the response of the molecule after evolving for a period $\tau$. Depending on the probe wavelength, different types of response will be monitored, including stimulated absorption/emission, photoelectron emission, and IR excitations.

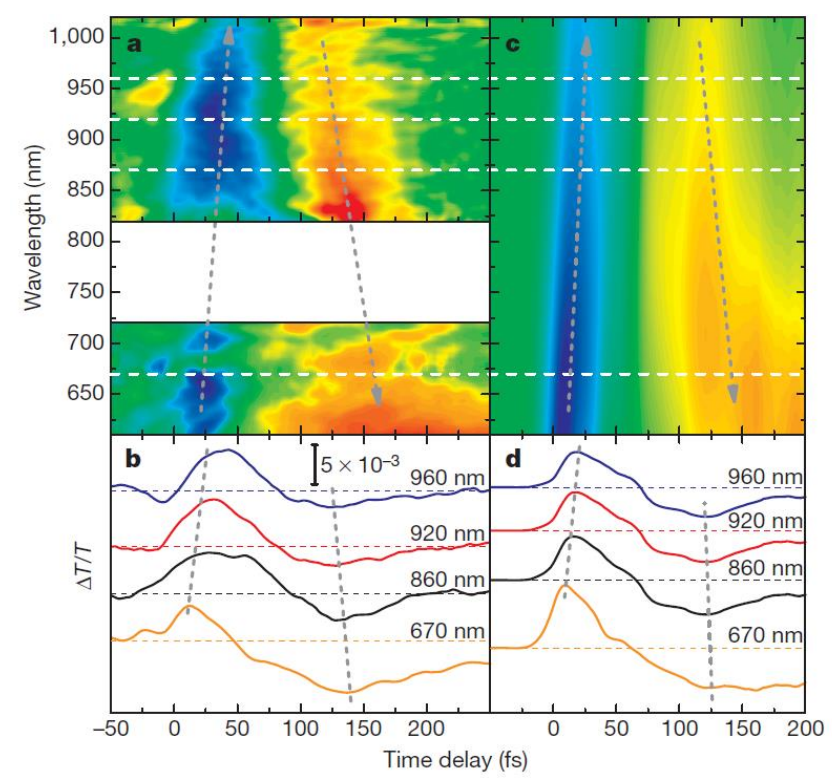

Fig. 9 Comparison between experimental (a) and TSH simulated (c) differential transmission as a function of the time delay and of the wavelength. Reproduced from Reprinted by permission from Macmillan Publishers Ltd: Ref. ${ }^{393}$, copyright 2010 .

The simplest approximation is to simulate time-resolved spectroscopy using NA-MQC dynamics is to use the nuclear ensemble approach discussed in Section 5.1, but for specific time intervals. In practical terms, after finishing the NA-MQC simulations, the results are split into time intervals, and spectra are built separately for each one. This procedure has been used, for instance, to simulate the time-resolved differential transmission in the retinal chromophore by Polli et al. ${ }^{393}$ (Fig. 9). In these simulations, for each 1-fs time interval, the absorption and emission probabilities from the active state into the other states were computed. Then, all contributions were added, and the result was convoluted with Gaussian functions to match the experimental 15-fs resolution.

NA-MQC dynamics has also been used as a basis for simulations of time-resolved photoelectron spectroscopy (TRPES). ${ }^{24}$, 216-217, 325, 389-390, 394-397 Bennett, Kowalewski, and Mukamel $^{24}$ have shown that the time-resolved case can still be written analogously to Eq. (93), but with the initial ensemble distribution given by the ensemble population $\eta_{I}^{\tau}(\mathbf{R})$ of state $I$ at the time $\tau$. In the same spirit as that of time-resolved stimulated emission, the dynamics results are 
split into time intervals, and the photoelectron is computed for each interval separately, to simulate the time delay between the photoexcitation and the photoionization. Thus, the TRPES becomes

$$
\Gamma\left(E_{b}, \tau\right)=\sum_{\kappa} \frac{1}{N_{p}} \sum_{n=1}^{N_{p}} \sigma_{I \kappa}\left(E_{b}, \mathbf{R}_{n}(\tau)\right) w_{r}\left[E_{b}-\Delta V_{I \kappa}\left(\mathbf{R}_{n}(\tau)\right)-E_{1}\right]
$$

where the binding energy $E_{b}=E_{1}+E_{2}-K_{e}$ is defined in terms of the two laser pulses, $E_{1}$ (pump) and $E_{2}$ (probe). Different from the steady result, in the TRPES, a significant fraction of electrons is ejected with low kinetic energies due to a rearrangement of the nuclear wave packet caused by its interaction with the probe pulse. ${ }^{389}$ For this reason, the sharp line shape $w_{s}$ adopted for the steady-state spectrum has been replaced by a rectangular function $w_{r}$ with a threshold at $E_{b}-\Delta V_{I \kappa}\left(\mathbf{R}_{n}(\tau)\right)-E_{1} \cdot{ }^{399}$ Simulations comparing the peaked vibrational background (PVB) model with $w_{s}$ and the constant vibrational background (CVB) model with $w_{r}$ are discussed in Ref. $^{390}$.

Going beyond the NEA, time-resolved spectrum can also be simulated from NA-MQC dynamics using more involved approaches. Fingerhut, Dorfman, and Mukamel, for instance, have developed a methodology for calculation of UV pump-IR probe spectra based on TSH. ${ }^{421}$ In their approach, the TSH results are used to reconstruct the excited-state vibrational Hamiltonian. Then, full quantum propagation of the Green's function is carried out to build the spectral signal.

Transient Raman spectra can be simulated following the protocol presented in Ref. ${ }^{422}$. The spectral signal is computed for each geometry in the ensemble generated from dynamics by computing the rank-2 static polarizability tensor of the electronic excited state. The calculation of such quantity, although still time-consuming, has been enabled by recent developments of analytical Hessians at (LR) TDDFT level. ${ }^{423}$

An excellent general discussion on the use of ensembles to compute time-resolved photoelectron, Auger-electron, and X-ray photon scattering spectroscopy can be found in Ref. ${ }^{24}$.

\subsubsection{Two-dimensional electronic spectrum}

Two-dimensional electronic spectroscopy (2DES) is a nonlinear optical technique, measuring the full nonlinear polarization of a quantum system in third order with respect to the 
field-matter interaction. ${ }^{424}$ In a typical experimental 2DES set up, ${ }^{23}$ the molecular system is excited by a sequence of three pulses, with relative delays $\tau_{1}$ and $\tau_{2}$. This pulse sequence creates a nonlinear polarization that emits a field delayed by $\tau_{3}$ after the third pulse. This field is measured, and the signal is Fourier transformed with respect to $\tau_{1}$ and $\tau_{3}$, for fixed $\tau_{2}$. The plot of the result as a function of the excitation frequency $\Omega_{1}$ and the detection frequency $\Omega_{2}$ is the $2 \mathrm{D}$ spectrum with the information on the third-order nonlinear optical response of the system.

Mukamel and co-authors have developed the sum-of-states (SOS) method, which allows computing general optical properties of many-electron systems. In particular, the third-order static polarizability can be obtained from a sum-of-states including the transition dipole moments and excitation energies between ground and excited states as well as between different excited states (see Eq. (4) of Ref. ${ }^{291}$ ). The SOS result has been recently explored by Garavelli's group to simulate 2DES based on QM/MM TSH dynamics. ${ }^{306,415,425}$ Although it has delivered promising results, the drawback of this approach is the enormous number of electronic states required in the simulation; 2DES for adenine, for instance, called for 150 states. ${ }^{426}$ Alternatively, 2DES simulated with either hierarchy equations of motion (HEOM) or numerical integration of Schrodinger equation (NISE) methods using MFE and TSH dynamics has been described in Refs. ${ }^{307,427}$.

\section{Software resources for NA-MQC dynamics}

With the increasing popularity of NA-MQC dynamics, different implementations of these methods have been made publicly available. These simulations need energies and classical forces (potential energy gradients), which are routinely obtained using standard electronic quantum chemistry (EQC) codes. Non-adiabatic couplings are available for several methods or numerically evaluated (Section 4.3). Most of the available codes implement one of these two strategies:

- Dedicated NA-MQC platforms fed by an interface to EQC programs.

- Standard EQC codes that have incorporated NA-MQC algorithms.

The first strategy involves some processor overload because frequent I/O associated with the execution of external programs, which are not always optimized to perform under the same conditions. The second strategy involves less overload but also limits simulations to those 
electronic methods implemented in the EQC code and usually provide limited options to control the simulations.

In Table 2, we provide a survey of several programs with available NA-MQC options.

Given the rapid development in the field, more than a list of all existing software- - which for sure would be outdated within a few months - we intend to highlight some of the offered flavors for NA-MQC simulations in a variety of implementations.

Table 2. Survey of NA-MQC implementations of NA-MQC in public software.

\begin{tabular}{|c|c|c|c|c|}
\hline Program & $\begin{array}{c}\text { Electronic structure } \\
\text { methods }\end{array}$ & NA-MQC method & Refs. & Website $^{b}$ \\
\hline \multicolumn{5}{|c|}{ Dedicated NA-MQC dynamics software } \\
\hline ANT & analytical PES & $\begin{array}{c}\text { FSSH, FSTU, FSTU/SD, } \\
\text { CSDM, MFE, } \\
\text { army ant tunnelling }\end{array}$ & 84 & comp.chem.umn.edu/ant \\
\hline COBRAMM & $\begin{array}{l}\text { MCSCF, } \\
\text { MRCI/OMx, } \\
\text { QM/MM }\end{array}$ & FSSH & $428-429$ & sites.google.com/site/cobrammhomepage \\
\hline DFTBABY & TD-(LC)-DFTB & FSSH & 430 & www.dftbaby.chemie.uni-wuerzburg.de \\
\hline JADE & $\begin{array}{l}\text { LR-TDDFT, } \\
\text { CIS, ADC(2) }\end{array}$ & FSSH & 431 & jade-package.github.io/JADE \\
\hline LIBRA & Analytical PES & $\begin{array}{l}\text { FSSH, GFSH, MSSH, MFE } \\
\text { (external fields) }\end{array}$ & 432 & $\frac{\text { github.com/Quantum-Dynamics- }}{\underline{\text { Hub/Libra-X }}}$ \\
\hline NA-ESMD & $\begin{array}{c}\text { CEO, } \\
\text { TDHF/semiempirical, } \\
\text { CIS/semiempirical }\end{array}$ & FSSH & 293 & Contact authors \\
\hline NEWTON-X & $\begin{array}{c}\text { MRCI, MR-AQCC, } \\
\text { MCSCF, ADC(2), CC2, } \\
\text { CIS, LR-TDDFT, XMS- } \\
\text { CASPT2, , TD-DFTB }{ }^{a} \\
\text { QM/MM, analytical PES, } \\
\text { user-defined PES }\end{array}$ & $\begin{array}{c}\text { FSSH } \\
\left(\mathrm{IC} \text { and } \mathrm{ISC}^{a}\right)\end{array}$ & $433-434$ & www.newtonx.org \\
\hline PYXAID & $\begin{array}{c}\text { RT-TDKS, } \\
\text { RT-SCC-DFTB }\end{array}$ & FSSH, DISH (external fields) & 73,351 & $\underline{\text { acsu.buffalo.edu/ alexeyak/pyxaid }}$ \\
\hline SHARC & $\begin{array}{c}\text { MCSCF, MRCI, } \\
\text { MS-CASPT2, ADC(2), } \\
\text { LR-TDDFT, analytical } \\
\text { PES }\end{array}$ & FSSH, SHARC & 129 & sharc-md.org \\
\hline \multicolumn{5}{|c|}{ Electronic structure software with NA-MQC options } \\
\hline CPMD & $\begin{array}{c}\text { LR-TDDFT, ROKS, } \\
\text { QM/MM }\end{array}$ & $\begin{array}{l}\text { FSSH, MFE, CT-MQC } \\
\text { (IC and ISC) }\end{array}$ & $\begin{array}{c}147,358,371 \\
435\end{array}$ & cpmd.org \\
\hline GAMESS $^{a}$ & CASSCF & AIMS & $177,436-437$ & msg.ameslab.gov/gamess \\
\hline $\mathrm{GPAW}^{a}$ & RT-TDKS & MFE & 342,438 & wiki.fysik.dtu.dk/gpaw \\
\hline CHEMSHELL $^{a}$ & MRCI/OMx & FSSH & 44,439 & chemshell.org \\
\hline MOLCAS & SA-CASSCF & FSSH & 440 & molcas.org \\
\hline MOLPRO & CASSCF, MS-CASPT2 & AIMS & 95,441 & molpro.net \\
\hline MOPAC $^{a}$ & FOMO-CI & $\begin{array}{l}\text { FSSH and AIMS } \\
\text { (IC and ISC) }\end{array}$ & $97,172,442$ & Contact authors \\
\hline OCTOPUS & RT-TDKS & MFE & 443 & gitlab.com/octopus-code \\
\hline TURBOMOLE & LR-TDDFT & FSSH & $444-445$ & $\underline{\text { turbomole.com }}$ \\
\hline
\end{tabular}


${ }^{a}$ Development version. ${ }^{b}$ All websites accessed on September 20, 2017.

The available implementations target at different kind of systems: small molecules and medium size molecules (mostly for organic chemistry and biomolecular applications), large systems with localized excited states, and large systems with delocalized excited states (usually nanomaterial applications). For the small and medium molecules, programs implementing NAMQC with the multi- and single-reference methods discussed in Section 4 are regularly used. When the electronic excitation is spatially constricted, these methods can be combined with force fields to describe large-scale systems within QM/MM frameworks. ${ }^{13,32,190,193,442,447-449}$ Strategies to ease the computational cost include the use parameterized electronic methods or further approximations to the NA-MQC methods. For nano-scaled materials, density functional methods (Section 4.2), especially in the RT-TDKS variant, emerges as the primary adopted approach.

Because of its algorithmic simplicity and robust results, TSH is by far the most popular NA-MQC scheme. Table 2 lists several programs whose the central focus is TSH. This is the case of NewtON-X, ${ }^{433-434}$ DFTBABy,${ }^{430}$ JADE, ${ }^{431}$ COBRAMM, ${ }^{428}$ PyXAID,${ }^{73,351}$, and SHARC. ${ }^{129}$ Moreover, there are publically available implementations for all main NA-MQC schemes, including MS and MFE.

Since 2007, we have been developing the NEWTON-X platform, dedicated to TSH using the interface strategy. ${ }^{433-434}$ NEWTON-X allows simulating the whole TSH dynamics, from the generation of initial conditions to the statistical analysis, with an extensive range of electronic structure methods, from MRCI and XMS-CAPT2 to LR-ADC(2) and LR-TD-DFTB, and model Hamiltonians (Tully 1D, ${ }^{43}$ 2D conical intersection, ${ }^{450}$ spin boson ${ }^{451}$ ). Several algorithms are available in the program, including local diabatization (Section 3.2.1), HST couplings with either OD or DD approaches (Section 4.3), and SDM decoherence corrections (Section 3.1.1). Moreover, NEWTON-X counts on modules for spectrum simulations based on the nuclear ensemble approach (Section 5), enabling simulations of steady and time-resolved absorption, emission, and photoionization spectra. ${ }^{390,406}$ A recently developed external code, PYSOC, enables calculations of spin-orbit couplings with LR-TDDFT. ${ }^{373}$

COBRAMM from Garavelli and coworkers is tailored to perform MCSCF/MM simulations of organic and biomolecular systems within the FSSH approximation. ${ }^{428-429}$ TSH within QM/MM 
partitions is also included in NEWTON-X, CPMD ${ }^{371}$ and in a development version of CHEMSHELL. ${ }^{44}$ 127, 173, 452

PYXAID $^{73,351}$ focuses on large condensed-matter systems with TSH based on RT-TDKS approach (Section 4.2.5). This program provides access to the several methods developed by Prezhdo's group. ${ }^{133}$ It implements the neglect of back-reaction approximation (NBRA; also known as Classical Path approximation), which considers that the nuclear dynamics is not strongly affected by the electronic dynamics and employ ground state trajectories as a proxy for the excited state dynamics. $^{73}$

SHARC, developed by González' group, delivers a platform for NA-MQC simulations considering internal conversion, spin-orbit, and radiation-matter couplings with the homonymous method (Section 3.3.1). ${ }^{129,169}$ This code allows using different ab initio approaches within TSH. ${ }^{169}$ The NA-MQC treatment of intersystem crossing is also implemented in a MoPAC's development version by Persico and Granucci ${ }^{172}$ with the FOMO-CI semiempirical method. ${ }^{97}$ It has also been recently implemented in NEWTON-X. ${ }^{178}$

A series of TSH implementations has been done with a focus on reducing computational costs. It includes the NA-ESMD code from Tretiak and collaborators, ${ }^{293}$ enabling TSH with CEO, CIS, and TDHF (Section 4.2.3) based on semiempirical Hamiltonians. ${ }^{293}$ Reduced computational costs are also achieved with TSH dynamics based on MRCI/OMx and FOMO-CI semiempirical methods (Section 4.1) implemented in development versions of CHEMSHELL and MOPAC mentioned above. PYXAID has an active strategy of cost reduction to enable NA-MQC for nanoscaled systems, with its single-determinant Kohn-Sham (Section 4.2.5) and NBRA approximations.

All implementations mentioned so far depend on explicit electronic structure calculations usually performed simultaneously to the trajectory propagation. The ANT code pursues a different strategy, offering TSH and MFE algorithms based on parameterized PES. ${ }^{453}$ ANT allows simulations with the several algorithms developed in Truhlar's group, including the fewest switches with time uncertainty (FSTU), FSTU with stochastic decoherence (FSTU/SD), and coherent switches with decay of mixing (CSDM). ${ }^{47,74-75}$ ANT also provides an implementation of the army ant tunneling method ${ }^{84-85}$ within the MFE approach. 
The LIBRA platform developed by Akimov enables a "do-it-yourself" strategy through an open-source library including modules to perform quantum and classical dynamics simulations. ${ }^{432}$ A large variety of algorithms is available, including modules to perform MFE and TSH (with FSSH, GFSH, and MSSH) based on $\triangle \mathrm{SCF}$; routines to deal with trivial crossings and decoherence; algorithms to compute various sorts of matrix elements with Heller Gaussians; and a number of model Hamiltonians (Tully models, spin-boson, superexchange, etc.).

Several electronic structure quantum chemistry programs provide NA-MQC options as a complementary feature. FSSH with LR-TDDFT limited to ground/first-excited states is available in TURBomole ${ }^{444}$ (see Section 4.2.3 for a discussion on the limitations of such approach). The code takes advantage of the analytical couplings developed by Send and Furche ${ }^{361}$ (see Section 4.3). NA-MQC with FSSH and the A-FSSH algorithms can be run with Q-CHEM 77-78, 446. MOLCAS $^{440}$ and MOLPRO ${ }^{95}$ have implemented FSSH and AIMS respectively. AIMS has also been implemented in development versions of MOPAC and GAMESS, including intersystem crossing. ${ }^{177}$, 436, 442 MFE with RT-TDKS methods is available in OcTOPUS ${ }^{443}$ and in a development version of GPAW. $^{342,438}$

Lying between dedicated NA-MQC dynamics programs and EQC programs with NAMQC options, CPMD is a Car-Parrinello dynamics code with its own electronic structure methods. It allows simulating MFE with RT-TDKS ${ }^{435}$ as well as TSH with ROKS ${ }^{358}$ and LR-TDDFT. ${ }^{371}$ CPMD implementation of TSH with LR-TDDFT includes several developments by Tavernelli and coworkers, including Landau-Zener probabilities, external fields, and intersystem crossing. ${ }^{171,184}$ CT-MQC has been recently implemented in a development version of CPMD. ${ }^{147}$

\section{$7 \quad$ The accuracy problem}

\subsection{How reliable is NA-MQC dynamics?}

The central problem faced by on-the-fly NA-MQC dynamics today is reliability. The high computational costs to simulate electronic structure on-the-fly has led to a systematic method downgrade, affecting the precision and accuracy of the results. Fig. 10 schematically outlines the main types of problems the simulations may encounter. 
To provide an estimate of the computational costs involved in NA-MQC dynamics, we may consider that the computational resources allocated to the simulations should be of the order of

$$
T_{\text {total }} \approx N_{\text {Trajectories }} T_{\text {Single Point }} \frac{\tau_{\text {chem process }}}{\Delta \tau}
$$

where $N_{\text {trajectories }}$ is the number of trajectories, $T_{\text {Single }}$ Point is the time to compute energies, gradients, and nonadiabatic couplings for a unique geometry, $\tau_{\text {chem process }}$ is the duration of the chemical process of interest, and $\Delta \tau$ is the integration time step for the classical equations of motion.

\section{Dynamics}

- Decoherence

- Nonlocal effects

- Super-exchange

- Trivial crossings

\section{Statistics}

- Number of trajectories

- Trajectory duration

- Time step

- Simple $x$ double ensemble

\section{Wavefunction}

- Incomplete/unbalanced electron correlation

- Multi x single reference

- Density functional approximations

- Double and higher excitations

Fig. 10 Some of the main source of problems that may affect the precision and accuracy of NA-MQC dynamics. In the diagram, they are grouped into three categories: Dynamics - related to approximation in the time propagation algorithm; Statistics - related to the diverse types of ensembles and samplings; and Wavefunction - related to the approximations in the electronic structure. Issues in Statistics mainly affect the precision of the simulations. The issues in Dynamics and Wavefunction affect the accuracy. The effects of the problems related to Wavefunction are usually more important than those related to Dynamics.

Typical figures for Eq. (98) would be to simulate 100 trajectories, with single point calculations taking 1 CPU.h each, aiming at to investigate a chemical process occurring within 1 
ps with dynamics integrated with 0.5 fs time step. For such a case, the computational resources required would amount to 200 thousand CPU.h.

Two main strategies have been followed to cope with such high computational costs. First, statistical ensembles are reduced to the minimum, which affects the precision of the calculations; and second, electronic structure methods are downgraded, which affects the accuracy of the simulations.

Concerning the reduction of ensembles, the maximum statistical error uncertainty associated with the ensemble of trajectories is roughly

$$
\varepsilon \approx 0.98 N_{\text {Trajectories }}^{-1 / 2}
$$

for $95 \%$ confidence interval. Thus, 100 trajectories will not allow resolving any process occurring with a frequency lower than $10 \%$. In fact, according to the statistical tests by Nelson et al. ${ }^{294} 400$ trajectories may be needed to obtain statistically converged time constants. (See Ref. ${ }^{85}$ for a discussion on how rare events may still be investigated in small ensembles.) An interesting example of the effect of the number of trajectories is given by Weingart et $a l^{454}$. Their TSH stimulation of azobenzene, based on 920 trajectories, was able to reveal strong time-dependent oscillations in the excited-state population not resolved by several other simulations of the same molecule, working with smaller ensembles.

In TSH, in particular, another kind of ensemble reduction is usually adopted. In principle, TSH should rely on a double-ensemble strategy: first, multiple trajectories must be started from the same phase-space initial point; then, the same procedure should be repeated from many points of the phase space representing the initial vibrational distribution of the molecule. This doubleensemble strategy is seldom used and, in most of the on-the-fly investigations, only a single trajectory is started from each phase-space initial point.

Ensemble reductions are also achieved via increase of integration time step and reduction of the total propagation time. In Section 3.2.1, we have already discussed how large computational steps may lead to wrong nonadiabatic distributions due to the shape of the nonadiabatic couplings. Additionally, it impacts the accuracy of the numerical integrators. Short trajectories, in turn, limit the type of processes that can be investigated to the ultrafast scale (hundreds of femtoseconds to few picoseconds). 
Even with all this limitation on ensembles, NA-MQC dynamics generates an enormous quantity of information. One of our more recent simulations (100 atoms, 50 trajectories, 3 ps/trajectory, 0.5 fs time step), ${ }^{322}$ for instance, have produced data in the order of Terabyte. This situation imposes significant challenges for analysis, which must be automatized, with clear quantitative classification criteria. ${ }^{455-457}$

The second strategy to reduce computational costs commonly adopted is to downgrade the electronic structure level. NA-MQC dynamics are often run with small double- $\zeta$ basis sets, with methods providing an incomplete treatment of electron correlation, as CASSCF (which misses dynamical electron correlation) or TDDFT (which misses nondynamical electron correlation).

To illustrate how this level downgrade impacts dynamics, take for instance the case of $9 \mathrm{H}-$ adenine in the gas phase (Fig. 11). TSH computed with ab-initio MXS-CASPT2, ${ }^{223}$ MRCI with single excitations, semi-empirical OM2/MRCI, linear-response ADC(2), and linear-response TDDFT provide very different results for the ultrafast deactivation behavior of this molecule. ${ }^{263}$ Note that despite the large ensemble of functionals, none of the TDDFT simulations have been able to predict the ultrafast behavior of adenine. All of them deliver too small ground state populations compared to the experimental result. In fact, the best result, obtained with BHLYP, results from an error trade-off, where an underestimation of the electron correlation in the computation of the excitation energy, compensates for the wrong behavior of the ground state along the relevant reaction paths. ${ }^{458}$

The methods based on wavefunction theory (WFT) reported in Fig. 11 (MXS-CASPT2, $\mathrm{ADC}(2)$, MRCIS, and OM2/MRCI) do relatively well in the $\mathrm{S}_{0}$ population prediction. Nevertheless, they all give the right answer for different reasons, as the distributions of the main regions of the intersection seam visited by each method (C2 puckering, C6 puckering, and N9-H dissociation) do not agree.

This methodological divergence is not restricted to adenine, and several other divergent cases have been reported as well. Dynamics of the protonated Schiff PSB3 is expected to follow different paths with TDDFT, CASSCF, and high-correlated methods. ${ }^{252,459-460} 2$-aminopyrimidine dynamics based on CASSCF follows different pathways, depending on how the active space is initially set up. ${ }^{197}$ Thymine dynamics delivers qualitatively different results with either CASSCF or $\operatorname{ADC}(2) .{ }^{255}$ 
When we become aware of all this methodological divergence, it is natural to ask where it comes from. Is it a consequence of the specific nonadiabatic dynamics algorithm, or is it due to the electronic structure method?

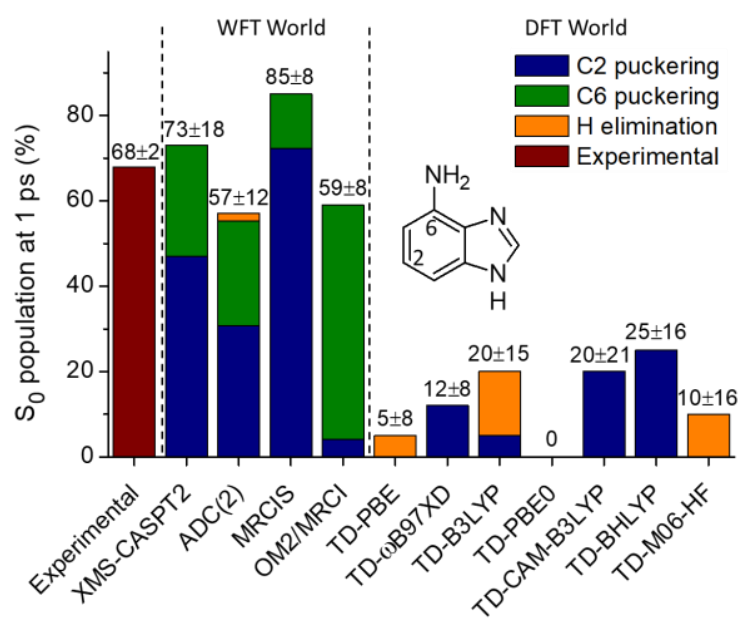

Fig. 11 Ground state population of 9H-adenine in the gas phase after 1 ps according to decoherence-corrected FSSH dynamics performed with different electronic structure methods based on wavefunction theory (WFT) and density functional theory (DFT). Experimental result from Ref. ${ }^{461}$. XMS-CASPT2 result from Ref. ${ }^{223}$. Figure adapted from Ref. ${ }^{263}$. Copyright 2014 American Chemical Society.

Following the analysis of Subotnik, Ouyang, and Landry of FSSH in the context of QCLE ${ }^{21}$ we may heuristically assume that if 1) nuclei have large momenta and 2) there are no significant recoherences and interferences between nuclear wave packets, a method like decoherence-corrected TSH will deliver qualitatively correct results. Admittedly, there are no practical criteria in place to quantify how large should be the momenta or how much interference is acceptable. Nevertheless, comparisons between decoherence-corrected TSH and exact results for model systems ${ }^{29,66,79,121,462}$ and MS results for atomistic simulations ${ }^{66}$ confirm the good agreement between these methods. A recent benchmark comparing TSH to a numerically exact spin-boson model has confirmed that FSSH, especially with decoherence corrections, provides accurate results over a wide range of parameters. ${ }^{462}$ Although encouraging, this good agreement should be taken cautiously, as the spin-boson Hamiltonian misses anharmonic effects and conical intersections. (Results for a spin-boson model reported in Ref. ${ }^{463}$, comparing MCTDH and MS on the one hand and TSH and MFE without decoherence corrections on the other, show that uncorrected TSH and MFE may fail for some coupling strength regimes and are sensitive to initial conditions.) 
Overall, the sharp differences pointed out above seem to be mostly caused by the electronic structure itself. Note, for instance, that all results in Fig. 11 were computed with the same surface hopping set up and still deliver different results, which indicates that the divergence is caused by the electronic structure method. Another example showing that comes from thymine: CASSCF dynamics, computed with either $\mathrm{MS}^{397}$ or $\mathrm{TSH},{ }^{464}$ predicts deactivation through the same pathways. We recognize, however, that the database for controlled comparisons between MS and TSH is still scarce ${ }^{66,463,465}$ due to the high costs of both types of simulations.

The robustness of a method like TSH is related to the fact that many of the ultrafastphotochemistry systems of interest have strong nonadiabatic couplings, in which the system hops down and moves away from the crossing. Under this condition, the hopping event does not depend on accurate coupling values, ${ }^{466}$ and errors due to multiple crossings ${ }^{66}$ do not build up. We already mentioned, there are cases, however, where decoherence and weak couplings are in play, and conventional TSH may not do well (see Sections 3.1.1 and 3.2.1).

Curiously, more than the dynamics method itself, the initial conditions selection may have a significant impact on the results of the dynamics. Ben-Nun and Martínez have observed thatdifferent from MS-TSH and MFE are sensitive to the initial conditions sampling method. ${ }^{463}$ In particular, when comparing TSH and MFE to MCTDH for a spin-boson model, they found out that the initial sampling with a quasi-classical distribution delivered better results than with a Wigner distribution. Note, however, that both TSH and MFE were not corrected for decoherence, a factor which may have a significant impact on processes with multiple population transfers. ${ }^{66} \mathrm{In}$ Ref. ${ }^{467}$, it is shown that the conventional approach of sampling initial conditions from snapshots of a thermal equilibrated ground-state dynamics to start the excited-state TSH may render artificially narrow initial coordinate distributions. This happens because the thermal energy at room temperature $\left(k_{B} T \sim 210 \mathrm{~cm}^{-1}\right)$ is much smaller than the vibrational zero-point energy of many of the normal modes. Tests for pyrrole comparing thermal and Wigner samplings showed that Wigner delivered superior results for absorption spectrum and internal conversion time constant. (See also Ref. ${ }^{41}$ for a discussion on the effect of the narrow thermal energy distribution on IR-excited dynamics.)

NA-MQC dynamics simulations have been widely focusing on sub-picosecond processes. We may expect, however, that longer processes taking place within few tens of picoseconds will 
soon become the new target, especially when discussing the competition between internal conversion and intersystem crossing. Then, new sources of inaccuracy, which are mostly neglected now, will become relevant. In particular, error accumulation in the integration of the quantum and classical equations and zero-point energy leakage in classical vibrational degrees of freedom ${ }^{92,468-}$ ${ }^{469}$ may become a serious concern.

The primary variable controlling the quality of the NA-MQC dynamics is the electronic structure method. The sensitivity of dynamics to the electronic structure has its roots in the nature of the excited states. The ground electronic state is usually energetically distant from any higher excited state, which renders well behaved potential energy surfaces, commonly with restricted diabatic character and mostly harmonic. (This is one of the reasons underlying the good general behavior of molecular mechanics.)

The excited states, however, lie close to each other. Thus, any minor nuclear displacement leads to a shift in the coupling between these states. Consequently, during dynamics in the excited states, even if it is restricted to a single surface, say $S_{1}$, the molecule will visit many different diabatic regions. A single bond stretching may change the surface character from $\pi \pi^{*}$ to $\pi \sigma^{*}$ for example. ${ }^{470}$

Currently, there is no available method able to describe all diabatic regions at the same footing. TDDFT with a hybrid functional will deliver excellent localized states and poor charge transfer ones; ${ }^{303}$ CASSCF will fairly predict the $n \pi *$ state but will overshoot the ionic $\pi \pi^{*}$ by 1 $\mathrm{eV}$ or more. ${ }^{195-196}$ This unbalancing in the description of different diabatic characters may artificially modulate the barriers on the excited state surfaces, forcing the dynamics to follow wrong pathways. Table 3 lists some of the problems that may occur in the NA-MQC simulations due to the approximations in the electronic structure methods.

The fact that there are no available, affordable methods that can provide a fully consistent description of the several regions of the configurational space is not limited to dynamics. It does equally affect static calculations as well (in the next section we will discuss an example of how static calculations have even led to the wrong interpretation of experimental results), although to a lesser extent, as the lower computational costs allow for better tuning of the levels.

Table 3. Problems that may occur in NA-MQC simulations due to approximations in the electronic structure method. 


\begin{tabular}{|c|c|c|}
\hline Problem & Methods affected & Solution / workaround \\
\hline Overshoot of ionic states ${ }^{195-196}$ & MCSCF, CASSCF & $\begin{array}{l}\text { Use fully correlated methods. Scaled } \\
\text { CASSCF }\end{array}$ \\
\hline Orbital exchange between subspaces & MCSCF, CASSCF & Enlarge active space \\
\hline Intruder states 471 & CASPT2 & Enlarge active space. Use level shifts \\
\hline $\begin{array}{l}\text { Unphysical responses when the difference } \\
\text { between the excitation energies of two states } \\
\text { matches the excitation of a third state }{ }^{287}\end{array}$ & Any linear-response method & $\begin{array}{l}\text { Avoid systems showing crossings } \\
\text { with the ground state }\end{array}$ \\
\hline $\begin{array}{c}\text { Numerical instabilities near crossings } \\
\text { between excited states }\end{array}$ & Linear-response CC & Use a Hermitian method like ADC \\
\hline $\begin{array}{l}\text { Numerical instabilities near crossings with the } \\
\text { ground state }\end{array}$ & $\begin{array}{l}\text { Single reference methods } \\
\text { (ADC, CC, TDDFT) }\end{array}$ & $\begin{array}{l}\text { Avoid systems showing crossing with } \\
\text { the ground state. Stop trajectory at the } \\
\text { crossing. } D_{1} \text { and } D_{2} \text { diagnostics for } \\
\text { MP and } C C^{284-285} \text { may help to detect } \\
\text { MR character of the ground state }\end{array}$ \\
\hline $\begin{array}{l}\text { Wrong dimensionality of intersections with } \\
\text { the ground state } 250,252\end{array}$ & $\begin{array}{l}\text { Methods for which only } \\
\text { excitation energies are } \\
\text { computed, like in linear } \\
\text { response. Also SS-CASPT2 }\end{array}$ & $\begin{array}{l}\text { Avoid systems showing crossing with } \\
\text { the ground state. Stop trajectories at } \\
\text { the crossing. Use } \mathrm{SF}^{258,472}\end{array}$ \\
\hline Negative excitations & $\begin{array}{l}\text { Methods for which only } \\
\text { excitation energies are } \\
\text { computed, like in linear } \\
\text { response. }\end{array}$ & $\begin{array}{l}\text { Stop trajectories at the crossing with } \\
\text { the ground state }\end{array}$ \\
\hline Wrong dissociation & Single reference methods & $\begin{array}{l}\text { TDA may help in TDDFT. }{ }^{253} \mathrm{D}_{1} \text { and } \\
\mathrm{D}_{2} \text { diagnostics for MP and CC } \mathrm{CC}^{284-285} \\
\text { may help to detect the problem in } \\
\text { ADC and CC }\end{array}$ \\
\hline Underestimated charge-transfer states ${ }^{303-304}$ & TDDFT & Use range-separated functionals 303 \\
\hline Missing double and higher excitations 295 & LR ADC(2), LR ALDA TDDFT & $\begin{array}{l}\text { Use another method to monitor higher } \\
\text { excitations. Use SF }\end{array}$ \\
\hline Underestimation of high-energy states ${ }_{313}^{310,312-}$ & LR TDDFT & $\begin{array}{l}\text { Use asymptotically corrected } \\
\text { functionals } 310\end{array}$ \\
\hline
\end{tabular}

This lack of methods also does not mean that there is nothing to do in the field, but to expect better and faster electronic structure methods to be developed. On the contrary, it is our opinion that a careful selection of methods, with a cross comparison between methodologies from different families (e.g., CASPT2 x TDDFT) and respect to the limits of each approximation will effectively allow to set up dynamics for most of the systems of interest.

For instance, single reference methods like linear response TDDFT and ADC(2) may provide a good description of the excited state dynamics of many systems. ${ }^{263,473}$ (See also Ref. ${ }^{28}$ for a specific discussion on NA-MQC dynamics with excited state DFT.) Because they account for dynamical electron correlation, they may perform even better than CASSCF, as long as the system is moving far from the intersection to the ground state. 
Nevertheless, given the disturbing divergent results discussed in this section, is there any way we could ensure that our NA-MQC simulations are not producing spurious results? This question will be addressed in the next section.

\subsection{Reaction paths or NA-MQC Dynamics: what's the best quantum chemistry we can do?}

The previous section may be led to somewhat pessimistic perspectives for the NA-MQC dynamics field. Let us show through an example that this does not need to be the case. NA-MQC dynamics can still play a significant role in quantum chemistry as long as its limits are respected.

A classical problem in photochemistry has been the double proton transfer in 7-azaindole (7AI) dimer. ${ }^{474}$ For over two decades, there has been a debate-sometimes with even some unusually harsh tones ${ }^{475}$ _on whether these transfers are concerted ${ }^{476}$ or stepwise $^{477}$ (see Fig. 12).

We have recently contributed to this field, by using TSH. ${ }^{280}$ The 7AI dimer dynamics, as set up in the experiments, should proceed through tunneling, which is not considered in TSH. For this reason, our simulations, in principle, were not intended to answer the concerted/stepwise question, but just to explore the configurational space. To our surprise, during these simulations, internal conversion to the ground state happening after the first proton transfer quenched every attempt at a stepwise transfer. It was evident then that stepwise transfers are impossible for this dimer either ballistically or via tunneling.

Why does this simple piece of information, which could have ended the debate years ago, was not revealed before? First, because most of the computational results describing the potential energy surfaces of the dimer were qualitatively wrong, starting with the CIS calculations that guided the experimental analysis of Zewail's group. ${ }^{474,478}$ It was only in 2006 that the right balance between the charge transfer and the local excitation regions of the $S_{1}$ surface was correctly described by the first time, thanks to CASPT2 calculations by Merchán and Serrano-Andrés. ${ }^{479}$ However, even then, the $\mathrm{S}_{1} / \mathrm{S}_{0}$ conical intersection went still unnoticed. 


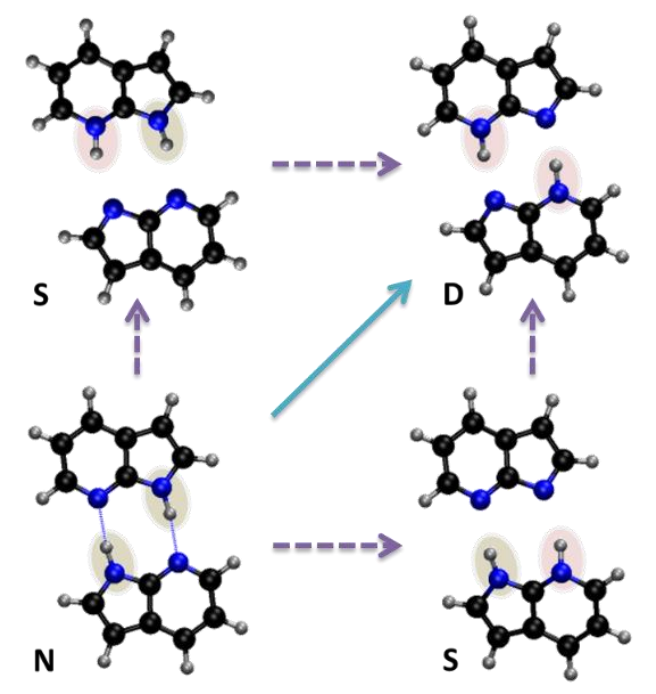

Fig. 12 7-Azaindole dimer. After photoexcitation, double proton transfer occurs. There is a long-standing debate whether it is concerted (dashed arrows) or concerted (solid arrow). Reprinted with permission from Ref. ${ }^{280}$. Copyright 2015 Royal Societ of Chemistry.

The crossing seam was overlooked because the dynamics of the 7-AI dimer was thought to be described by a couple of hydrogen transfer coordinates dictated by chemical intuition. However, excited-state dynamics is precisely where chemical intuition may fail. Our dynamics simulations allowed the dimer to explore the configurational space entirely, without any bias towards particular coordinates. This is the reason it could reveal the intersection.

This example allows us to draw some general thoughts on the role of NA-MQC dynamics and perspectives for the near future of the field.

To a large extent, the work on computational chemistry consists of determining the fate of molecular systems based on the energies, energy gradients, and state couplings as a function of the nuclear coordinates. Two of the main ways of fulfilling this goal is either by computing reaction paths along specific coordinates or running dynamics.

On the one hand, reaction paths computation allows applying the best electronic structure methods, at the cost of restricting them to a biased subset of nuclear coordinates, usually dictated by chemical intuition. On the other hand, NA-MQC dynamics suffers from an extreme electronic method downgrade but does not impose any restriction on the nuclear coordinates.

Thus, NA-MQC dynamics is a tool to explore the configurational space of molecular systems, to let the critical reaction coordinates to reveal themselves. It must, however, be followed 
by rigorous evaluation of the reaction pathways along these coordinates, using high-level electronic structure methods. This second step should become mandatory in the future if we want to ensure the quality of our predictions.

\section{Which method to use?}

In this review, we have discussed an enormous amount of methods for NA-MQC dynamics. For a non-specialist, this may lead to a feeling of lost among too many options. (An instructive organogram highlighting the relations between different TSH algorithms is given in the review by Wang et al. ${ }^{133}$ The reader may also profit from several topic reviews published in the last years. They are listed in Table 4.) Therefore, we would like to end this work with general advice on which methods to choose.

Table 4. Survey of recent reviews on the topics discussed or related to this work.

\begin{tabular}{|c|c|}
\hline Authors, Reference & Topic \\
\hline Yarkony, $2011^{480}$ & Nonadiabatic theory \\
\hline Yonehara et al., $2011^{30}$ & Nonadiabatic theory \\
\hline Matsika and Krause, $2011^{481}$ & Nonadiabatic theory: conical intersections \\
\hline Blumberger, $2015^{482}$ & Nonadiabatic theory: electron transfer (biosystems) \\
\hline Oberhofer et al., $2017^{483}$ & Nonadiabatic theory: electron transfer (organic solids) \\
\hline de Carvalho et al., $2014^{16}$ & NA-MQC dynamics \\
\hline Persico and Granucci, $2014^{92}$ & NA-MQC dynamics \\
\hline Tavernelli, $2015^{31}$ & NA-MQC dynamics \\
\hline Tully, $2012^{466}$ & NA-MQC dynamics: perspectives \\
\hline Akimov and Prezhdo, $2015^{484}$ & NA-MQC dynamics: large-scale systems \\
\hline Akimov et al., $2013^{485}$ & NA-MQC applications: photocatalysis \\
\hline Nelson et al., $2014^{12}$ & NA-MQC applications: conjugated materials \\
\hline Kilina et al., $2015^{486}$ & NA-MQC applications: organic and semiconductor nanostructures \\
\hline Brunk and Rothlisberger, $2015^{13}$ & NA-MQC applications: biological systems \\
\hline Wang et al., $2015^{14}$ & NA-MQC applications: nanoscale interfaces \\
\hline Hammes-Schiffer, $2015^{15}$ & NA-MQC applications: tunneling \\
\hline Makhov et al., 201722 & MFE \\
\hline Barbatti, $2011^{27}$ & $\mathrm{TSH}$ \\
\hline Mai et al., 2015 & TSH: arbitrary couplings \\
\hline Subotnik et al., $2016^{29}$ & TSH: decoherence corrections \\
\hline Wang et al., $2016^{133}$ & TSH: new algorithms \\
\hline Barbatti and Crespo-Otero, $2016^{391}$ & TSH with DFT \\
\hline Weingart, $2017^{193}$ & TSH with QM/MM \\
\hline Curchod and Martínez, $2018^{7}$ & Ab initio nonadiabatic quantum molecular dynamics \\
\hline Richings et al., $2015^{159}$ & vMCG \\
\hline Casida and Huix-Rotllant, 2012289 & Electronic structure: TDDFT \\
\hline Huix-Rotllant et al., $2016^{472}$ & Electronic structure: conical intersections in DFT \\
\hline Dreuw and Wormit, $2015^{266}$ & Electronic structure: ADC \\
\hline Sneskov and Christiansen, $2012^{260}$ & Electronic structure: $\mathrm{CC}$ \\
\hline Elstner and Seifert, $2014^{317}$ & Electronic structure: DFTB \\
\hline Szalay et al., $2011^{209}$ & Electronic structure: MC and MR methods \\
\hline
\end{tabular}


Naturally, the adequate method will depend on each situation, balancing between how much computational cost may be afforded, which kind of processes needs to be investigated, and which level of precision and accuracy is desired. From our experience, for investigation of ultrafast (few picoseconds) internal conversion, one of the best balances between these variables may be achieved with TSH in adiabatic representation, using FSSH probabilities (Eq. (15)), obtained via local diabatization (Eq. (33)), and corrected for decoherence with the SDM method (Eq. (26)). In the case of intersystem crossing processes, TSH based on spin-adiabatic representation like in SHARC (Section 3.3) should be adopted. If during the time evolution the system recurrently returns to the strong NAC region, MS may be required.

Needless to emphasize that this choice is entirely subjective, and most likely other specialists would deliver a different prescription. Nevertheless, we may point out that this methodological protocol offers the following advantages: 1) it is well tested for many systems and its shortcomings are well known; 2) it is available in diverse public dynamics packages; 3 ) in the case of TSH, it fixes some of the most significant problems of this method (decoherence, trivial crossings, weak couplings) at a minimal cost.

While we may prescribe a preferred protocol for NA-MQC dynamics, we cannot do so for the electronic structure method. A large variety of such methods has been tested and used with NA-MQC dynamics; the approaches span multireference and single-reference, $a b$ initio and semiempirical, wave-function-based and density-functional methods. Each has its domain of validity and computational costs, the variables that should be balanced when vouching for or against any of them. We have outlined in Section 7.1 some general criteria for choosing the electronic structure method, but the decision still needs to be done case by case, under scrutiny.

As a last thought, NA-MQC dynamics is a powerful tool in quantum chemistry, opening several avenues for the research of ultrafast nonadiabatic processes. The intense development of methods and programs in the field in the last years has been continuously expanding its domains to new phenomena, larger systems, and longer times. As soon as we finally tame all the hurdles with precision and accuracy, we may contemplate an era when time-resolved spectroscopy in silico and in vitro will develop hand in hand. 


\section{Acknowledgements}

MB work was supported by Excellence Initiative of Aix-Marseille University (A*MIDEX) and the project Equip@ Meso (ANR-10-EQPX-29-01), both funded by the French Government "Investissements d'Avenir" program. MB also acknowledges funding from HPC resources from GENCI-CINES (2017-A0010810012) and of the WSPLIT project (ANR-17-CE05-0005-01). RCO acknowledges the support from the School of Biological and Chemical Sciences at the Queen Mary University of London. The authors thank helpful discussions with N. Ferré, M. Filatov, E. Fromager, G. Groenhof, M. Huix-Rotllant, R. Lindh, and J. W. Park. They are also grateful for comments on the manuscript by A. Akimov, B. Churchod, M. Dommett, L. González, G. Granucci, S. Mai, F. Plasser, J. Subotnik, R. Szabla, I. Tavernelli, S. Tretiak, and O. Weingart.

\section{References}

(1) Martens, C. C.; Fang, J.-Y. Semiclassical-Limit Molecular Dynamics on Multiple Electronic Surfaces. J. Chem. Phys. 1997, 106, 4918-4930.

(2) Kapral, R.; Ciccotti, G. Mixed Quantum-Classical Dynamics. J. Chem. Phys. 1999, 110, 8919-8929.

(3) Mac Kernan, D.; Ciccotti, G.; Kapral, R. Trotter-Based Simulation of Quantum-Classical Dynamics. J. Phys. Chem. B 2008, 112, 424-432.

(4) Stock, G.; Thoss, M. Semiclassical Description of Nonadiabatic Quantum Dynamics. Phys. Rev. Lett. 1997, 78, 578-581.

(5) Thoss, M.; Stock, G. Mapping Approach to the Semiclassical Description of Nonadiabatic Quantum Dynamics. Phys. Rev. A 1999, 59, 64-79.

(6) Ben-Nun, M.; Quenneville, J.; Martínez, T. J. Ab Initio Multiple Spawning: Photochemistry from First Principles Quantum Molecular Dynamics. J. Phys. Chem. A 2000, 104, 5161-5175.

(7) Curchod, B. F.; Martínez, T. J. Ab Initio Nonadiabatic Quantum Molecular Dynamics. Chem. Rev. 2018, 118, 3305-3336.

(8) Tavernelli, I. Ab Initio-driven Trajectory-Based Nuclear Quantum Dynamics in Phase Space. Phys. Rev. A 2013, 87, 042501.

(9) Curchod, B. F. E.; Tavernelli, I.; Rothlisberger, U. Trajectory-Based Solution of the Nonadiabatic Quantum Dynamics Equations: An on-the-Fly Approach for Molecular Dynamics Simulations. Phys. Chem. Chem. Phys. 2011, 13, 3231-3236.

(10) Agostini, F.; Min, S. K.; Abedi, A.; Gross, E. K. U. Quantum-Classical Nonadiabatic Dynamics: Coupled- vs Independent-Trajectory Methods. J. Chem. Theory Comput. 2016, 12, 2127-2143.

(11) Pratihar, S.; Ma, X.; Homayoon, Z.; Barnes, G. L.; Hase, W. L. Direct Chemical Dynamics Simulations. J. Am. Chem. Soc. 2017, 139, 3570-3590.

(12) Nelson, T.; Fernandez-Alberti, S.; Roitberg, A. E.; Tretiak, S. Nonadiabatic Excited-State Molecular Dynamics: Modeling Photophysics in Organic Conjugated Materials. Acc. Chem. Res. 2014, 47, 1155-1164. 
(13) Brunk, E.; Rothlisberger, U. Mixed Quantum Mechanical/Molecular Mechanical Molecular Dynamics Simulations of Biological Systems in Ground and Electronically Excited States. Chem. Rev. 2015, 115, 6217-6263.

(14) Wang, L.; Long, R.; Prezhdo, O. V. Time-Domain Ab Initio Modeling of Photoinduced Dynamics at Nanoscale Interfaces. Annu. Rev. Phys. Chem. 2015, 66, 549-579.

(15) Hammes-Schiffer, S. Proton-Coupled Electron Transfer: Moving Together and Charging Forward. J. Am. Chem. Soc. 2015, 137, 8860-8871.

(16) de Carvalho, F.; Bouduban, M.; Curchod, B.; Tavernelli, I. Nonadiabatic Molecular Dynamics Based on Trajectories. Entropy 2014, 16, 62-85.

(17) Stock, G.; Thoss, M., Classical Description of Nonadiabatic Quantum Dynamics. In Adv. Chem. Phys., John Wiley \& Sons, Inc.: 2005; Vol. 131, pp 243-375.

(18) Kelly, A.; Markland, T. E. Efficient and Accurate Surface Hopping for Long Time Nonadiabatic Quantum Dynamics. J. Chem. Phys. 2013, 139, 014104.

(19) Worth, G. A.; Robb, M. A.; Lasorne, B. Solving the Time-Dependent Schrödinger Equation for Nuclear Motion in One Step: Direct Dynamics of Non-Adiabatic Systems. Mol. Phys. 2008, 106, 2077-2091.

(20) Vacher, M.; Bearpark, M. J.; Robb, M. A. Direct methods for non-adiabatic dynamics: connecting the single-set variational multi-configuration Gaussian (vMCG) and Ehrenfest perspectives. Theor. Chem. Acc. 2016, 135, 187.

(21) Subotnik, J. E.; Ouyang, W.; Landry, B. R. Can We Derive Tully's Surface-Hopping Algorithm from the Semiclassical Quantum Liouville Equation? Almost, but Only with Decoherence. J. Chem. Phys. 2013, 139, 214107.

(22) Makhov, D.; Symonds, C.; Fernandez-Alberti, S.; Shalashilin, D. Ab Initio Quantum Direct Dynamics Simulations of Ultrafast Photochemistry with Multiconfigurational Ehrenfest Approach. Chem. Phys. 2017, 493, 200-218.

(23) Fuller, F. D.; Ogilvie, J. P. Experimental Implementations of Two-Dimensional Fourier Transform Electronic Spectroscopy. Annu. Rev. Phys. Chem. 2015, 66, 667-690.

(24) Bennett, K.; Kowalewski, M.; Mukamel, S. Probing Electronic and Vibrational Dynamics in Molecules by Time-Resolved Photoelectron, Auger-Electron, and X-Ray Photon Scattering Spectroscopy. Faraday Discuss. 2015, 177, 405-428.

(25) Stolow, A.; Underwood, J. G., Time-Resolved Photoelectron Spectroscopy of Nonadiabatic Dynamics in Polyatomic Molecules. In Adv. Chem. Phys., John Wiley \& Sons, Inc.: 2008; pp 497-584.

(26) Zewail, A. H. Femtochemistry. Past, present, and future. Pure Appl. Chem. 2000, 72, 22192231.

(27) Barbatti, M. Nonadiabatic Dynamics with Trajectory Surface Hopping Method. WIREs: Comp. Mol. Sci. 2011, 1, 620-633.

(28) Barbatti, M.; Crespo-Otero, R. Surface Hopping Dynamics with DFT Excited States. Top. Curr. Chem. 2015, 368, 415-444.

(29) Subotnik, J. E.; Jain, A.; Landry, B.; Petit, A.; Ouyang, W.; Bellonzi, N. Understanding the Surface Hopping View of Electronic Transitions and Decoherence. Annu. Rev. Phys. Chem. 2016, 67, 387-417.

(30) Yonehara, T.; Hanasaki, K.; Takatsuka, K. Fundamental Approaches to Nonadiabaticity: Toward a Chemical Theory beyond the Born-Oppenheimer Paradigm. Chem. Rev. 2011, 112, 499542. 
(31) Tavernelli, I. Nonadiabatic Molecular Dynamics Simulations: Synergies between Theory and Experiments. Acc. Chem. Res. 2015, 48, 792-800.

(32) Virshup, A. M.; Punwong, C.; Pogorelov, T. V.; Lindquist, B. A.; Ko, C.; Martinez, T. J. Photodynamics in Complex Environments: $\mathrm{Ab}$ Initio Multiple Spawning Quantum Mechanical/Molecular Mechanical Dynamics. J. Phys. Chem. B 2009, 113, 3280-3291.

(33) Herman, M. F.; Arce, J. C. A Semiclassical Surface Hopping Formalism for SolventInduced Vibrational Relaxation. Chem. Phys. 1994, 183, 335-350.

(34) Hammes-Schiffer, S.; Tully, J. C. Proton Transfer in Solution: Molecular Dynamics with Quantum Transitions. J. Chem. Phys. 1994, 101, 4657-4667.

(35) Tully, J. C. Mixed Quantum-Classical Dynamics. Faraday Discuss. 1998, 110, 407-419.

(36) Swope, W. C.; Andersen, H. C.; Berens, P. H.; Wilson, K. R. A Computer-Simulation Method for the Calculation of Equilibrium-Constants for the Formation of Physical Clusters of Molecules - Application to Small Water Clusters. J. Chem. Phys. 1982, 76, 637-649.

(37) Barbatti, M.; Shepard, R.; Lischka, H., Computational and Methodological Elements for Nonadiabatic Trajectory Dynamics Simulations of Molecules. In Conical Intersections: Theory, Computation and Experiment, Domcke, W.; Yarkony, D. R.; Koppel, H., Eds. World Scientific: Singapore, 2010; pp 415-462.

(38) Vacher, M.; Mendive-Tapia, D.; Bearpark, M. J.; Robb, M. A. The second-order Ehrenfest method. Theor. Chem. Acc. 2014, 133, 1505.

(39) Parandekar, P. V.; Tully, J. C. Mixed Quantum-Classical Equilibrium. J. Chem. Phys. 2005, 122, 094102.

(40) Bastida, A.; Cruz, C.; Zúñiga, J.; Requena, A.; Miguel, B. A Modified Ehrenfest Method that Achieves Boltzmann Quantum State Populations. Chem. Phys. Lett. 2006, 417, 53-57.

(41) Bastida, A.; Zúñiga, J.; Requena, A.; Miguel, B. Full Quantum Vibrational Simulation of the Relaxation of the Cyanide Ion in Water Using the Ehrenfest Method with Quantum Corrections. J. Chem. Phys. 2008, 129, 154501.

(42) Hammes-Schiffer, S.; Tully, J. C. Proton-Transfer in Solution - Molecular-Dynamics with Quantum Transitions. J. Chem. Phys. 1994, 101, 4657-4667.

(43) Tully, J. C. Molecular-Dynamics with Electronic-Transitions. J. Chem. Phys. 1990, 93, 1061-1071.

(44) Fabiano, E.; Keal, T. W.; Thiel, W. Implementation of Surface Hopping Molecular Dynamics Using Semiempirical Methods. Chem. Phys. 2008, 349, 334-347.

(45) Pechukas, P. Time-Dependent Semiclassical Scattering Theory. II. Atomic Collisions. Phys. Rev. 1969, 181, 174-185.

(46) Herman, M. F.; Kluk, E. A Semiclasical Justification for the Use of Non-Spreading Wavepackets in Dynamics Calculations. Chem. Phys. 1984, 91, 27-34.

(47) Jasper, A. W.; Stechmann, S. N.; Truhlar, D. G. Fewest-Switches with Time Uncertainty: A Modified Trajectory Surface-Hopping Algorithm with Better Accuracy for Classically Forbidden Electronic Transitions. J. Chem. Phys. 2002, 116, 5424-5431.

(48) Kapral, R. Surface Hopping from the Perspective of Quantum-classical Liouville Dynamics. Chem. Phys. 2016, 481, 77-83.

(49) Martens, C. C. Surface Hopping by Consensus. J. Phys. Chem. Lett. 2016, 7, 2610-2615.

(50) Landry, B. R.; Falk, M. J.; Subotnik, J. E. Communication: The Correct Interpretation of Surface Hopping Trajectories: How to Calculate Electronic Properties. J. Chem. Phys. 2013, 139, 211101. 
(51) Schmidt, J. R.; Parandekar, P. V.; Tully, J. C. Mixed Quantum-Classical Equilibrium: Surface Hopping. J. Chem. Phys. 2008, 129, 044104.

(52) Jain, A.; Herman, M. F.; Ouyang, W.; Subotnik, J. E. Surface Hopping, Transition State Theory and Decoherence. I. Scattering Theory and Time-Reversibility. J. Chem. Phys. 2015, 143, 134106.

(53) Ben-Nun, M.; Martínez, T. J., Ab Initio Quantum Molecular Dynamics. In Adv. Chem. Phys., John Wiley \& Sons, Inc.: 2002; Vol. 121, pp 439-512.

(54) Yang, S.; Martínez, T. J., Ab Initio Multiple Spawning: First Principles Dynamics Around Conical Intersections. In Conical Intersections, World Scientific: 2012; pp 347-374.

(55) Martinez, T. J.; Levine, R. D. Dynamics of the Collisional Electron Transfer and Femtosecond Photodissociation of $\mathrm{NaI}$ on Ab Initio Electronic Energy Curves. Chem. Phys. Lett. 1996, 259, 252-260.

(56) Smith, B. R.; Bearpark, M. J.; Robb, M. A.; Bernardi, F.; Olivucci, M. Classical Wavepacket Dynamics through a Conical Intersection - Application to the $S_{1} / S_{0}$ Photochemistry of Benzene. Chem. Phys. Lett. 1995, 242, 27-32.

(57) Thompson, A. L.; Punwong, C.; Martínez, T. J. Optimization of Width Parameters for Quantum Dynamics with Frozen Gaussian Basis Sets. Chem. Phys. 2010, 370, 70-77.

(58) Hack, M. D.; Wensmann, A. M.; Truhlar, D. G.; Ben-Nun, M.; Martínez, T. J. Comparison of Full Multiple Spawning, Trajectory Surface Hopping, and Converged Quantum Mechanics for Electronically Nonadiabatic Dynamics. J. Chem. Phys. 2001, 115, 1172-1186.

(59) Saita, K.; Shalashilin, D. V. On-the-Fly Ab Initio Molecular Dynamics with Multiconfigurational Ehrenfest Method. J. Chem. Phys. 2012, 137, 22A506-8.

(60) Makhov, D. V.; Glover, W. J.; Martinez, T. J.; Shalashilin, D. V. Ab Initio Multiple Cloning Algorithm for Quantum Nonadiabatic Molecular Dynamics. J. Chem. Phys. 2014, 141, 054110.

(61) Prezhdo, O. V.; Rossky, P. J. Evaluation of Quantum Transition Rates from QuantumClassical Molecular Dynamics Simulations. J. Chem. Phys. 1997, 107, 5863-5878.

(62) Schwartz, B. J.; Bittner, E. R.; Prezhdo, O. V.; Rossky, P. J. Quantum Decoherence and the Isotope Effect in Condensed Phase Nonadiabatic Molecular Dynamics Simulations. J. Chem. Phys. 1996, 104, 5942-5955.

(63) Subotnik, J. E.; Shenvi, N. Decoherence and Surface Hopping: When Can Averaging over Initial Conditions Help Capture the Effects of Wave Packet Separation? J. Chem. Phys. 2011, 134, 244114.

(64) Granucci, G.; Persico, M. Critical Appraisal of the Fewest Switches Algorithm for Surface Hopping. J. Chem. Phys. 2007, 126, 134114-11.

(65) Nelson, T.; Fernandez-Alberti, S.; Roitberg, A. E.; Tretiak, S. Nonadiabatic Excited-State Molecular Dynamics: Treatment of Electronic Decoherence. J. Chem. Phys. 2013, 138, 22411113.

(66) Granucci, G.; Persico, M.; Zoccante, A. Including Quantum Decoherence in Surface Hopping. J. Chem. Phys. 2010, 133, 134111.

(67) Ouyang, W.; Subotnik, J. E. Estimating the Entropy and Quantifying the Impurity of a Swarm of Surface-Hopping Trajectories: A New Perspective on Decoherence. J. Chem. Phys. 2014, 140, 204102.

(68) Bastida, A.; Cruz, C.; Zúñiga, J.; Requena, A. Collective probabilities algorithm for surface hopping calculations. J. Chem. Phys. 2003, 119, 6489-6499. 
(69) Akimov, A. V.; Long, R.; Prezhdo, O. V. Coherence penalty functional: A simple method for adding decoherence in Ehrenfest dynamics. J. Chem. Phys. 2014, 140, 194107.

(70) Schwartz, B. J.; Rossky, P. J. Aqueous Solvation Dynamics with a Quantum Mechanical Solute: Computer Simulation Studies of the Photoexcited Hydrated Electron. J. Chem. Phys. 1994, 101, 6902-6916.

(71) Jain, A.; Alguire, E.; Subotnik, J. E. An Efficient, Augmented Surface Hopping Algorithm that Includes Decoherence for Use in Large-Scale Simulations. J. Chem. Theory Comput. 2016, 12, 5256-5268.

(72) Jaeger, H. M.; Fischer, S.; Prezhdo, O. V. Decoherence-Induced Surface Hopping. J. Chem. Phys. 2012, 137, 22A545.

(73) Akimov, A. V.; Prezhdo, O. V. Advanced Capabilities of the PYXAID Program: Integration Schemes, Decoherence Effects, Multiexcitonic States, and Field-Matter Interaction. $J$. Chem. Theory Comput. 2014, 10, 789-804.

(74) Zhu, C. Y.; Nangia, S.; Jasper, A. W.; Truhlar, D. G. Coherent Switching with Decay of Mixing: An Improved Treatment of Electronic Coherence for Non-Born-Oppenheimer Trajectories. J. Chem. Phys. 2004, 121, 7658-7670.

(75) Zhu, C.; Jasper, A. W.; Truhlar, D. G. Non-Born-Oppenheimer Trajectories with SelfConsistent Decay of Mixing. J. Chem. Phys. 2004, 120, 5543-5557.

(76) Zhu, C.; Jasper, A. W.; Truhlar, D. G. Non-Born-Oppenheimer Liouville-von Neumann Dynamics. Evolution of a Subsystem Controlled by Linear and Population-Driven Decay of Mixing with Decoherent and Coherent Switching. J. Chem. Theory Comput. 2005, 1, 527-540.

(77) Landry, B. R.; Subotnik, J. E. How to Recover Marcus Theory with Fewest Switches Surface Hopping: Add Just a Touch of Decoherence. J. Chem. Phys. 2012, 137, 22A513.

(78) Shenvi, N.; Subotnik, J. E.; Yang, W. Simultaneous-Trajectory Surface Hopping: A Parameter-Free Algorithm for Implementing Decoherence in Nonadiabatic Dynamics. J. Chem. Phys. 2011, 134, 144102.

(79) Gao, X.; Thiel, W. Non-Hermitian Surface Hopping. Phys. Rev. E 2017, 95, 013308.

(80) Ha, J.-K.; Lee, I. S.; Min, S. K. Surface Hopping Dynamics beyond Nonadiabatic Couplings for Quantum Coherence. J. Phys. Chem. Lett. 2018, 9, 1097-1104.

(81) Ben-Nun, M.; Martínez, T. J. A Multiple Spawning Approach to Tunneling Dynamics. $J$. Chem. Phys. 2000, 112, 6113-6121.

(82) Makhov, D. V.; Martinez, T. J.; Shalashilin, D. V. Toward Fully Quantum Modelling of Ultrafast Photodissociation Imaging Experiments. Treating Tunnelling in the Ab Initio Multiple Cloning Approach. Faraday Discuss. 2016, 194, 81-94.

(83) Nangia, S.; Jasper, A. W.; III, T. F. M.; Truhlar, D. G. Army Ants Algorithm for Rare Event Sampling of Delocalized Nonadiabatic Transitions by Trajectory Surface Hopping and the Estimation of Sampling Errors by the Bootstrap Method. J. Chem. Phys. 2004, 120, 3586-3597.

(84) Zheng, J.; Xu, X.; Meana-Paneda, R.; Truhlar, D. G. Army Ants Tunneling for Classical Simulations. Chem. Sci. 2014, 5, 2091-2099.

(85) Zheng, J.; Meana-Pañeda, R.; Truhlar, D. G. Including Tunneling in Non-BornOppenheimer Simulations. J. Phys. Chem. Lett. 2014, 5, 2039-2043.

(86) Xu, X.; Zheng, J.; Yang, K. R.; Truhlar, D. G. Photodissociation Dynamics of Phenol: Multistate Trajectory Simulations including Tunneling. J. Am. Chem. Soc. 2014, 136, 1637816386.

(87) Parrinello, M.; Rahman, A. Study of an F Center in Molten KCl. J. Chem. Phys. 1984, 80, 860-867. 
(88) Lu, J.; Zhou, Z. Path Integral Molecular Dynamics with Surface Hopping for Thermal Equilibrium Sampling of Nonadiabatic Systems. J. Chem. Phys. 2017, 146, 154110.

(89) Krishna, V. Path Integral Formulation for Quantum Nonadiabatic Dynamics and the Mixed Quantum Classical Limit. J. Chem. Phys. 2007, 126, 134107.

(90) Shushkov, P.; Li, R.; Tully, J. C. Ring Polymer Molecular Dynamics with Surface Hopping. J. Chem. Phys. 2012, 137, 22 A549.

(91) Lu, J.; Zhou, Z. Accelerated Sampling by Infinite Swapping of Path Integral Molecular Dynamics with Surface Hopping. J. Chem. Phys. 2018, 148, 064110.

(92) Persico, M.; Granucci, G. An Overview of Nonadiabatic Dynamics Simulations Methods, with Focus on the Direct Approach Versus the Fitting of Potential Energy Surfaces. Theor. Chem. Acc. 2014, 133, 1526.

(93) Barbatti, M.; Belz, S.; Leibscher, M.; Lischka, H.; Manz, J. Sensitivity of Femtosecond Quantum Dynamics and Control with Respect to Non-Adiabatic Couplings: Model Simulations for the Cis-Trans Isomerization of the Dideuterated Methaniminium Cation. Chem. Phys. 2008, $350,145-153$.

(94) Wang, L.; Prezhdo, O. V. A Simple Solution to the Trivial Crossing Problem in Surface Hopping. J. Phys. Chem. Lett. 2014, 5, 713-719.

(95) Levine, B. G.; Coe, J. D.; Virshup, A. M.; Martinez, T. J. Implementation of Ab Initio Multiple Spawning in the MOLPRO Quantum Chemistry Package. Chem. Phys. 2008, 347, 3-16.

(96) Spörkel, L.; Thiel, W. Adaptive Time Steps in Trajectory Surface Hopping Simulations. J. Chem. Phys. 2016, 144, 194108.

(97) Granucci, G.; Persico, M.; Toniolo, A. Direct Semiclassical Simulation of Photochemical Processes with Semiempirical Wave Functions. J. Chem. Phys. 2001, 114, 10608-10615.

(98) Plasser, F.; Granucci, G.; Pittner, J.; Barbatti, M.; Persico, M.; Lischka, H. Surface Hopping Dynamics Using a Locally Diabatic Formalism: Charge Transfer in the Ethylene Dimer Cation and Excited State Dynamics in the 2-Pyridone Dimer. J. Chem. Phys. 2012, 137, 22A51413.

(99) Fernandez-Alberti, S.; Roitberg, A. E.; Nelson, T.; Tretiak, S. Identification of Unavoided Crossings in Nonadiabatic Photoexcited Dynamics Involving Multiple Electronic States in Polyatomic Conjugated Molecules. J. Chem. Phys. 2012, 137, 014512.

(100) Meek, G. A.; Levine, B. G. Evaluation of the Time-Derivative Coupling for Accurate Electronic State Transition Probabilities from Numerical Simulations. J. Phys. Chem. Lett. 2014, $5,2351-2356$.

(101) Desouter-Lecomte, M.; Lorquet, J. C. Nonadiabatic Interactions in Unimolecular Decay. IV. Transition Probability as a Function of the Massey Parameter. J. Chem. Phys. 1979, 71, 43914403.

(102) Shenvi, N.; Tully, J. C. Nonadiabatic Dynamics at Metal Surfaces: Independent Electron Surface Hopping with Phonon and Electron Thermostats. Faraday Discuss. 2012, 157, 325-335.

(103) Shenvi, N.; Roy, S.; Tully, J. C. Nonadiabatic Dynamics at Metal Surfaces: IndependentElectron Surface Hopping. J. Chem. Phys. 2009, 130, 174107.

(104) Roy, S.; Shenvi, N.; Tully, J. C. Dynamics of Open-Shell Species at Metal Surfaces. J. Phys. Chem. C 2009, 113, 16311-16320.

(105) Wang, L.; Beljonne, D. Flexible Surface Hopping Approach to Model the Crossover from Hopping to Band-like Transport in Organic Crystals. J. Phys. Chem. Lett. 2013, 4, 1888-1894.

(106) Ruckenbauer, M.; Barbatti, M.; Sellner, B.; Muller, T.; Lischka, H. Azomethane: Nonadiabatic Photodynamical Simulations in Solution. J. Phys. Chem. A 2010, 114, 12585-12590. 
(107) Spencer, J.; Gajdos, F.; Blumberger, J. FOB-SH: Fragment Orbital-Based Surface Hopping for Charge Carrier Transport in Organic and Biological Molecules and Materials. J. Chem. Phys. 2016, 145, 064102.

(108) Akimov, A. V. Nonadiabatic Molecular Dynamics with Tight-Binding Fragment Molecular Orbitals. J. Chem. Theory Comput. 2016, 12, 5719-5736.

(109) Goyal, P.; Schwerdtfeger, C. A.; Soudackov, A. V.; Hammes-Schiffer, S. Nonadiabatic Dynamics of Photoinduced Proton-Coupled Electron Transfer in a Solvated Phenol-Amine Complex. J. Phys. Chem. B 2015, 119, 2758-2768.

(110) Venkataraman, C.; Soudackov, A. V.; Hammes-Schiffer, S. Dynamics of Photoinduced Proton-Coupled Electron Transfer at Molecule-Semiconductor Interfaces: A Reduced Density Matrix Approach. J. Phys. Chem. C 2009, 114, 487-496.

(111) Morelli, J.; Hammes-Schiffer, S. Surface Hopping and Fully Quantum Dynamical Wavepacket Propagation on Multiple Coupled Adiabatic Potential Surfaces for Proton Transfer Reactions. Chem. Phys. Lett. 1997, 269, 161-170.

(112) Kelly, A.; van Zon, R.; Schofield, J.; Kapral, R. Mapping Quantum-Classical Liouville Equation: Projectors and Trajectories. J. Chem. Phys. 2012, 136, 084101.

(113) Kuntz, P. J.; Kendrick, J.; Whitton, W. N. Surface-Hopping Trajectory Calculations of Collision-Induced Dissociation Processes with and without Charge Transfer. Chem. Phys. 1979, $38,147-160$.

(114) Ali, D. P.; Miller, W. H. Effect of Electronic Transition Dynamics on Iodine Atom Recombination in Liquids. J. Chem. Phys. 1983, 78, 6640-6645.

(115) Hu, W.; Lendvay, G.; Maiti, B.; Schatz, G. C. Trajectory Surface Hopping Study of the $\mathrm{O}\left(\mathrm{P}_{3}\right)$ Plus Ethylene Reaction Dynamics. J. Phys. Chem. A 2008, 112, 2093-2103.

(116) Yu, L.; Xu, C.; Lei, Y.; Zhu, C.; Wen, Z. Trajectory-Based Nonadiabatic Molecular Dynamics without Calculating Nonadiabatic Coupling in the Avoided Crossing Case: Trans $\leftrightarrow$ Cis Photoisomerization in Azobenzene. Phys. Chem. Chem. Phys. 2014, 16, 25883-25895.

(117) Yue, L.; Yu, L.; Xu, C.; Lei, Y.; Liu, Y.; Zhu, C. Benchmark Performance of Global Switching versus Local Switching for Trajectory Surface Hopping Molecular Dynamics Simulation: Cis $\leftrightarrow$ Trans Azobenzene Photoisomerization. ChemPhysChem 2017, 18, 1274-1287. (118) Zener, C. Non-Adiabatic Crossing of Energy Levels. Proceedings of the Royal Society of London. Series A 1932, 137, 696-702.

(119) Wittig, C. The Landau-Zener Formula. J. Phys. Chem. B 2005, 109, 8428-8430.

(120) Belyaev, A. K.; Lasser, C.; Trigila, G. Landau-Zener Type Surface Hopping Algorithms. J. Chem. Phys. 2014, 140, 224108.

(121) Xie, W.; Domcke, W. Accuracy of Trajectory Surface-Hopping Methods: Test for a TwoDimensional Model of the Photodissociation of Phenol. J. Chem. Phys. 2017, 147, 184114.

(122) Belyaev, A. K.; Lebedev, O. V. Nonadiabatic Nuclear Dynamics of Atomic Collisions Based on Branching Classical Trajectories. Phys. Rev. A 2011, 84, 014701.

(123) Zhu, C.; Nakamura, H. The Two-state Linear Curve Crossing Problems Revisited. III. Analytical Approximations for Stokes Constant and Scattering Matrix: Nonadiabatic Tunneling Case. J. Chem. Phys. 1993, 98, 6208-6222.

(124) Tully, J. C.; Preston, R. K. Trajectory Surface Hopping Approach to Nonadiabatic Molecular Collisions: Reaction of $\mathrm{H}^{+}$with $\mathrm{D}_{2}$. J. Chem. Phys. 1971, 55, 562-572.

(125) Blais, N. C.; Truhlar, D. G. Trajectory-Surface-Hopping Study of $\mathrm{Na}\left(3 \mathrm{p}^{2} \mathrm{P}\right)+\mathrm{H}_{2} \rightarrow$ $\mathrm{Na}\left(3 \mathrm{~s}^{2} \mathrm{~S}^{\prime}+\mathrm{H}_{2}\left(v^{\prime}, \mathrm{j}^{\prime}, \theta\right)\right.$. J. Chem. Phys. 1983, 79, 1334-1342. 
(126) Herman, M. F. A Semiclassical Surface Hopping Propagator for Nonadiabatic Problems. J. Chem. Phys. 1995, 103, 8081-8097.

(127) Fabiano, E.; Groenhof, G.; Thiel, W. Approximate Switching Algorithms for Trajectory Surface Hopping. Chem. Phys. 2008, 351, 111-116.

(128) Mitrić, R.; Petersen, J.; Bonačić-Koutecký, V. Laser-Field-Induced Surface-Hopping Method for the Simulation and Control of Ultrafast Photodynamics. Phys. Rev. A 2009, 79, 1-6.

(129) Richter, M.; Marquetand, P.; González-Vázquez, J.; Sola, I.; González, L. SHARC: Ab Initio Molecular Dynamics with Surface Hopping in the Adiabatic Representation Including Arbitrary Couplings. J. Chem. Theory Comput. 2011, 7, 1253-1258.

(130) Akimov, A. V.; Trivedi, D.; Wang, L.; Prezhdo, O. V. Analysis of the Trajectory Surface Hopping Method from the Markov State Model Perspective. J. Phys. Soc. Jpn. 2015, 84, 094002. (131) Wang, L.; Trivedi, D.; Prezhdo, O. V. Global Flux Surface Hopping Approach for Mixed Quantum-Classical Dynamics. J. Chem. Theory Comput. 2014, 10, 3598-3605.

(132) Akimov, A. V.; Prezhdo, O. V. Second-Quantized Surface Hopping. Phys. Rev. Lett. 2014, $113,153003$.

(133) Wang, L.; Akimov, A.; Prezhdo, O. V. Recent Progress in Surface Hopping: 2011-2015. J. Phys. Chem. Lett. 2016, 7, 2100-2112.

(134) Worth, G. A.; Cederbaum, L. S. Beyond Born-Oppenheimer: Molecular Dynamics through a Conical Intersection. Annu. Rev. Phys. Chem. 2004, 55, 127-158.

(135) Abedi, A.; Maitra, N. T.; Gross, E. K. U. Exact Factorization of the Time-Dependent Electron-Nuclear Wave Function. Phys. Rev. Lett. 2010, 105, 123002.

(136) Abedi, A.; Maitra, N. T.; Gross, E. K. U. Correlated Electron-Nuclear Dynamics: Exact Factorization of the Molecular Wavefunction. J. Chem. Phys. 2012, 137, 22A530.

(137) Hunter, G. Conditional probability amplitudes in wave mechanics. Int. J. Quantum Chem. 1975, $9,237-242$.

(138) Curchod, B. F. E.; Agostini, F.; Gross, E. K. U. An Exact Factorization Perspective on Quantum Interferences in Nonadiabatic Dynamics. J. Chem. Phys. 2016, 145, 034103.

(139) Requist, R.; Tandetzky, F.; Gross, E. K. U. Molecular Geometric Phase from the Exact Electron-Nuclear Factorization. Phys. Rev. A 2016, 93, 042108.

(140) Min, S. K.; Abedi, A.; Kim, K. S.; Gross, E. K. U. Is the Molecular Berry Phase an Artifact of the Born-Oppenheimer Approximation? Phys. Rev. Lett. 2014, 113, 263004.

(141) Agostini, F.; Abedi, A.; Suzuki, Y.; Min, S. K.; Maitra, N. T.; Gross, E. K. U. The Exact Forces on Classical Nuclei in Non-Adiabatic Charge Transfer. J. Chem. Phys. 2015, 142, 084303. (142) Eich, F. G.; Agostini, F. The Adiabatic Limit of the Exact Factorization of the ElectronNuclear Wave Function. J. Chem. Phys. 2016, 145, 054110.

(143) Agostini, F.; Abedi, A.; Suzuki, Y.; Gross, E. K. U. Mixed Quantum-Classical Dynamics on the Exact Time-Dependent Potential Energy Surface: A Fresh Look at Non-Adiabatic Processes. Mol. Phys. 2013, 111, 3625-3640.

(144) Agostini, F.; Abedi, A.; Gross, E. K. U. Classical Nuclear Motion Coupled to Electronic Non-Adiabatic Transitions. J. Chem. Phys. 2014, 141, 214101.

(145) Ali, A.; Federica, A.; Gross, E. K. U. Mixed Quantum-Classical Dynamics from the Exact Decomposition of Electron-Nuclear Motion. EPL (Europhysics Letters) 2014, 106, 33001.

(146) Min, S. K.; Agostini, F.; Gross, E. K. U. Coupled-Trajectory Quantum-Classical Approach to Electronic Decoherence in Nonadiabatic Processes. Phys. Rev. Lett. 2015, 115, 073001. 
(147) Min, S. K.; Agostini, F.; Tavernelli, I.; Gross, E. K. U. Ab Initio Nonadiabatic Dynamics with Coupled Trajectories: A Rigorous Approach to Quantum (De)Coherence. J. Phys. Chem. Lett. 2017, 8, 3048-3055.

(148) Curchod, B. F. E.; Agostini, F. On the Dynamics through a Conical Intersection. J. Phys. Chem. Lett. 2017, 8, 831-837.

(149) Gorshkov, V. N.; Tretiak, S.; Mozyrsky, D. Semiclassical Monte-Carlo Approach for Modelling Non-Adiabatic Dynamics in Extended Molecules. Nat Commun 2013, 4, 2144.

(150) White, A.; Tretiak, S.; Mozyrsky, D. Coupled Wave-Packets for Non-Adiabatic Molecular Dynamics: A Generalization of Gaussian Wave-Packet Dynamics to Multiple Potential Energy Surfaces. Chem. Sci. 2016, 7, 4905-4911.

(151) Heller, E. J. Frozen Gaussians: A Very Simple Semiclassical Approximation. J. Chem. Phys. 1981, 75, 2923-2931.

(152) Worth, G. A.; Hunt, P.; Robb, M. A. Nonadiabatic Dynamics: A Comparison of Surface Hopping Direct Dynamics with Quantum Wavepacket Calculations. J. Phys. Chem. A 2003, 107, 621-631.

(153) Worth, G. A.; Meyer, H. D.; Köppel, H.; Cederbaum, L. S.; Burghardt, I. Using the MCTDH Wavepacket Propagation Method to Describe Multimode Non-Adiabatic Dynamics. Int. Rev. Phys. Chem. 2008, 27, 569-606.

(154) Shalashilin, D. V. Multiconfigurational Ehrenfest Approach to Quantum Coherent Dynamics in Large Molecular Systems. Faraday Discuss. 2011, 153, 105-116.

(155) Kondorskiy, A.; Nakamura, H. Semiclassical Theory of Electronically Nonadiabatic Chemical Dynamics: Incorporation of the Zhu-Nakamura Theory into the Frozen Gaussian Propagation Method. J. Chem. Phys. 2004, 120, 8937-8954.

(156) Garashchuk, S.; Dell'Angelo, D.; Rassolov, V. A. Dynamics in the Quantum/Classical Limit Based on Selective Use of the Quantum Potential. J. Chem. Phys. 2014, 141, 234107.

(157) Gu, B.; Garashchuk, S. Quantum Dynamics with Gaussian Bases Defined by the Quantum Trajectories. J. Phys. Chem. A 2016, 120, 3023-3031.

(158) Burghardt, I.; Meyer, H.-D.; Cederbaum, L. S. Approaches to the Approximate Treatment of Complex Molecular Systems by the Multiconfiguration Time-Dependent Hartree Method. $J$. Chem. Phys. 1999, 111, 2927-2939.

(159) Richings, G. W.; Polyak, I.; Spinlove, K. E.; Worth, G. A.; Burghardt, I.; Lasorne, B. Quantum Dynamics Simulations Using Gaussian Wavepackets: The vMCG Method. Int. Rev. Phys. Chem. 2015, 34, 269-308.

(160) Harvey, J. N. Understanding the Kinetics of Spin-Forbidden Chemical Reactions. Phys. Chem. Chem. Phys. 2007, 9, 331-343.

(161) Bai, S.; Barbatti, M. Divide-to-Conquer: A Kinetic Model for Singlet Oxygen Photosensitization. J. Chem. Theory Comput. 2017, 13, 5528-5538.

(162) Zaari, R. R.; Varganov, S. A. Nonadiabatic Transition State Theory and Trajectory Surface Hopping Dynamics: Intersystem Crossing Between ${ }^{3} \mathrm{~B}_{1}$ and ${ }^{1} \mathrm{~A}_{1}$ States of $\mathrm{SiH}_{2}$. J. Phys. Chem. A 2015, 119, 1332-1338.

(163) Helgaker, T.; Uggerud, E.; Jensen, H. J. A. Integration of the classical equations of motion on ab initio molecular potential energy surfaces using gradients and Hessians: application to translational energy release upon fragmentation. Chem. Phys. Lett. 1990, 173, 145-150.

(164) Lingerfelt, D. B.; Williams-Young, D. B.; Petrone, A.; Li, X. Direct Ab Initio (Meta)Surface-Hopping Dynamics. J. Chem. Theory Comput. 2016, 12, 935-945. 
(165) Laio, A.; Parrinello, M. Escaping Free-Energy Minima. Proc. Natl. Acad. Sci. USA 2002, 99, 12562-12566.

(166) Nijamudheen, A.; Akimov, A. V. Excited-State Dynamics in Two-Dimensional Heterostructures: $\mathrm{SiR} / \mathrm{TiO}_{2}$ and $\mathrm{GeR} / \mathrm{TiO}_{2}(\mathrm{R}=\mathrm{H}, \mathrm{Me})$ as Promising Photocatalysts. J. Phys. Chem. C 2017, 121, 6520-6532.

(167) Akimov, A. V. Stochastic and Quasi-Stochastic Hamiltonians for Long-Time Nonadiabatic Molecular Dynamics. J. Phys. Chem. Lett. 2017, 8, 5190-5195.

(168) Pollum, M.; Jockusch, S.; Crespo-Hernández, C. E. 2,4-Dithiothymine as a Potent UVA Chemotherapeutic Agent. J. Am. Chem. Soc. 2014, 136, 17930-17933.

(169) Mai, S.; Marquetand, P.; González, L. A General Method to Describe Intersystem Crossing Dynamics in Trajectory Surface Hopping. Int. J. Quantum Chem. 2015, 115, 1215-1231.

(170) Franco de Carvalho, F.; Curchod, B. F. E.; Penfold, T. J.; Tavernelli, I. Derivation of SpinOrbit Couplings in Collinear Linear-Response TDDFT: A Rigorous Formulation. J. Chem. Phys. 2014, 140, 144103.

(171) Franco de Carvalho, F.; Tavernelli, I. Nonadiabatic Dynamics with Intersystem Crossings: A Time-Dependent Density Functional Theory Implementation. J. Chem. Phys. 2015, 143, 224105.

(172) Granucci, G.; Persico, M.; Spighi, G. Surface Hopping Trajectory Simulations with SpinOrbit and Dynamical Couplings. J. Chem. Phys. 2012, 137, 22 A501.

(173) Cui, G.; Thiel, W. Generalized Trajectory Surface-Hopping Method for Internal Conversion and Intersystem Crossing. J. Chem. Phys. 2014, 141, 124101.

(174) Martínez-Fernández, L.; González, L.; Corral, I. An Ab Initio Mechanism for Efficient Population of Triplet States in Cytotoxic Sulfur Substituted DNA Bases: The Case of 6Thioguanine. Chem. Commun. 2012, 48, 2134-2136.

(175) Carbogno, C.; Behler, J.; Reuter, K.; Gross, A. Signatures of Nonadiabatic $\mathrm{O}_{2}$ Dissociation at Al(111): First-Principles Fewest-Switches Study. Phys. Rev. B 2010, 81, 035410.

(176) Curchod, B. F. E.; Rauer, C.; Marquetand, P.; González, L.; Martínez, T. J. Communication: GAIMS-Generalized Ab Initio Multiple Spawning for Both Internal Conversion and Intersystem Crossing Processes. J. Chem. Phys. 2016, 144, 101102.

(177) Fedorov, D. A.; Pruitt, S. R.; Keipert, K.; Gordon, M. S.; Varganov, S. A. Ab Initio Multiple Spawning Method for Intersystem Crossing Dynamics: Spin-Forbidden Transitions between ${ }^{3} \mathrm{~B}_{1}$ and ${ }^{1} \mathrm{~A}_{1}$ States of $\mathrm{GeH}_{2}$. J. Phys. Chem. A 2016, 120, 2911-2919.

(178) Pederzoli, M.; Pittner, J. A New Approach to Molecular Dynamics with Non-adiabatic and Spin-orbit Effects with Applications to QM/MM Simulations of Thiophene and Selenophene. $J$. Chem. Phys. 2017, 146, 114101.

(179) Spighi, G.; Gaveau, M.-A.; Mestdagh, J.-M.; Poisson, L.; Soep, B. Gas Phase Dynamics of Triplet Formation in Benzophenone. Phys. Chem. Chem. Phys. 2014, 16, 9610-9618.

(180) Favero, L.; Granucci, G.; Persico, M. Surface Hopping Investigation of Benzophenone Excited State Dynamics. Phys. Chem. Chem. Phys. 2016, 18, 10499-10506.

(181) Marazzi, M.; Mai, S.; Roca-Sanjuán, D.; Delcey, M. G.; Lindh, R.; González, L.; Monari, A. Benzophenone Ultrafast Triplet Population: Revisiting the Kinetic Model by Surface-Hopping Dynamics. J. Phys. Chem. Lett. 2016, 7, 622-626.

(182) Jones, G. A.; Acocella, A.; Zerbetto, F. On-the-Fly, Electric-Field-Driven, Coupled Electron-Nuclear Dynamics. J. Phys. Chem. A 2008, 112, 9650-9656.

(183) Fischer, M.; Handt, J.; Schmidt, R. Nonadiabatic Quantum Molecular Dynamics with Hopping. I. General Formalism and Case Study. Phys. Rev. A 2014, 90, 012525. 
(184) Tavernelli, I.; Curchod, B. F. E.; Rothlisberger, U. Mixed Quantum-Classical Dynamics with Time-Dependent External Fields: A Time-Dependent Density-Functional-Theory Approach. Phys. Rev. A 2010, 81, 052508.

(185) Han, Y.; Meng, Q.; Rasulev, B.; May, P. S.; Berry, M. T.; Kilin, D. S. Photofragmentation of the Gas-Phase Lanthanum Isopropylcyclopentadienyl Complex: Computational Modeling vs Experiment. J. Phys. Chem. A 2015, 119, 10838-10848.

(186) Chen, J.; Meng, Q.; Stanley May, P.; Berry, M. T.; Kilin, D. S. Time-Dependent ExcitedState Molecular Dynamics of Photodissociation of Lanthanide Complexes for Laser-Assisted Metal-Organic Chemical Vapour Deposition. Mol. Phys. 2014, 112, 508-517.

(187) Petersen, J.; Mitrić, R. Electronic Coherence within the Semiclassical Field-Induced Surface Hopping Method: Strong Field Quantum Control in $\mathrm{K}_{2}$. Phys. Chem. Chem. Phys. 2012, 14, 8299-306.

(188) Röhr, M. I. S.; Petersen, J.; Wohlgemuth, M.; Bonačić-Koutecký, V.; Mitrić, R. Nonlinear Absorption Dynamics Using Field-Induced Surface Hopping: Zinc Porphyrin in Water. ChemPhysChem 2013, 14, 1377-1386.

(189) Bajo, J. J.; González-Vázquez, J.; Sola, I. R.; Santamaria, J.; Richter, M.; Marquetand, P.; González, L. Mixed Quantum-Classical Dynamics in the Adiabatic Representation to Simulate Molecules Driven by Strong Laser Pulses. J. Phys. Chem. A 2012, 116, 2800-2807.

(190) Luk, H. L.; Feist, J.; Toppari, J. J.; Groenhof, G. Multiscale Molecular Dynamics Simulations of Polaritonic Chemistry. J. Chem. Theory Comput. 2017, 13, 4324-4335.

(191) Mignolet, B.; Curchod, B. F. E.; Martínez, T. J. Communication: XFAIMS—EXxternal Field Ab Initio Multiple Spawning for Electron-Nuclear Dynamics Triggered by Short Laser Pulses. J. Chem. Phys. 2016, 145, 191104.

(192) Mennucci, B., QM/MM Approaches for the Modeling of Photoinduced Processes in Biological Systems. In Quantum Modeling of Complex Molecular Systems, Rivail, J.-L.; RuizLopez, M.; Assfeld, X., Eds. Springer International Publishing: Cham, 2015; pp 325-342.

(193) Weingart, O. Combined Quantum and Molecular Mechanics (QM/MM) Approaches to Simulate Ultrafast Photodynamics in Biological Systems. Curr. Org. Chem. 2017, 21, 586-601.

(194) Schmidt, M. W.; Gordon, M. S. The Construction and Interpretation of MCSCF Wavefunctions. Annu. Rev. Phys. Chem. 1998, 49, 233-266.

(195) Angeli, C. On the Nature of the $\pi \rightarrow \pi^{*}$ Ionic Excited States: The V State of Ethene as a Prototype. J. Comput. Chem. 2009, 30, 1319-1333.

(196) Wu, W.; Zhang, H.; Braïda, B.; Shaik, S.; Hiberty, P. The V State of Ethylene: Valence Bond Theory Takes up the Challenge. Theor. Chem. Acc. 2014, 133, 1-13.

(197) Szymczak, J. J.; Barbatti, M.; Lischka, H. Influence of the Active Space on CASSCF Nonadiabatic Dynamics Simulations. Int. J. Quantum Chem. 2011, 111, 3307-3315.

(198) Sellner, B.; Barbatti, M.; Müller, T.; Domcke, W.; Lischka, H. Ultrafast Non-Adiabatic Dynamics of Ethylene Including Rydberg States. Mol. Phys. 2013, 111, 2439-2450.

(199) Keller, S. F.; Reiher, M. Determining Factors for the Accuracy of DMRG in Chemistry. CHIMIA International Journal for Chemistry 2014, 68, 200-203.

(200) Booth, G. H.; Alavi, A. Approaching Chemical Accuracy Using Full ConfigurationInteraction Quantum Monte Carlo: A Study of Ionization Potentials. J. Chem. Phys. 2010, 132, 174104.

(201) Booth, G. H.; Gruneis, A.; Kresse, G.; Alavi, A. Towards an Exact Description of Electronic Wavefunctions in Real Solids. Nature 2013, 493, 365-370. 
(202) Song, C.; Wang, L.-P.; Martínez, T. J. Automated Code Engine for Graphical Processing Units: Application to the Effective Core Potential Integrals and Gradients. J. Chem. Theory Comput. 2016, 12, 92-106.

(203) Titov, A. V.; Ufimtsev, I. S.; Luehr, N.; Martinez, T. J. Generating Efficient Quantum Chemistry Codes for Novel Architectures. J. Chem. Theory Comput. 2013, 9, 213-221.

(204) Hohenstein, E. G.; Bouduban, M. E. F.; Song, C.; Luehr, N.; Ufimtsev, I. S.; Martínez, T. J. Analytic First Derivatives of Floating Occupation Molecular Orbital-Complete Active Space Configuration Interaction on Graphical Processing Units. J. Chem. Phys. 2015, 143, 014111014111.

(205) Hohenstein, E. G.; Luehr, N.; Ufimtsev, I. S.; Martínez, T. J. An Atomic Orbital-Based Formulation of the Complete Active Space Self-Consistent Field Method on Graphical Processing Units. J. Chem. Phys. 2015, 142, 224103-224103.

(206) Frutos, L. M.; Andruniow, T.; Santoro, F.; Ferre, N.; Olivucci, M. Tracking the ExcitedState Time Evolution of the Visual Pigment with Multiconfigurational Quantum Chemistry. Proc. Natl. Acad. Sci. USA 2007, 104, 7764-7769.

(207) Snyder, J. W.; Parrish, R. M.; Martínez, T. J. a-CASSCF: An Efficient, Empirical Correction for SA-CASSCF To Closely Approximate MS-CASPT2 Potential Energy Surfaces. $J$. Phys. Chem. Lett. 2017, 8, 2432-2437.

(208) Werner, H.-J.; Knowles, P. J. An Efficient Internally Contracted Multiconfiguration-Reference Configuration Interaction Method. J. Chem. Phys. 1988, 89, 5803-5814.

(209) Szalay, P. G.; Müller, T.; Gidofalvi, G.; Lischka, H.; Shepard, R. Multiconfiguration SelfConsistent Field and Multireference Configuration Interaction Methods and Applications. Chem. Rev. 2011, 112, 108-181.

(210) Chattopadhyay, S.; Chaudhuri, R. K.; Mahapatra, U. S.; Ghosh, A.; Ray, S. S. StateSpecific Multireference Perturbation Theory: Development and Present Status. Wiley Interdisciplinary Reviews: Computational Molecular Science 2016, 6, 266-291.

(211) Roca-Sanjuán, D.; Aquilante, F.; Lindh, R. Multiconfiguration Second-Order Perturbation Theory Approach to Strong Electron Correlation in Chemistry and Photochemistry. Wiley Interdisciplinary Reviews: Computational Molecular Science 2012, 2, 585-603.

(212) Lischka, H.; Dallos, M.; Shepard, R. Analytic MRCI Gradient for Excited States: Formalism and Application to the $n-\pi^{*}$ Valence- and $n-(3 s, 3 p)$ Rydberg States of Formaldehyde. Mol. Phys. 2002, 100, 1647-1658.

(213) Dallos, M.; Lischka, H.; Shepard, R.; Yarkony, D. R.; Szalay, P. G. Analytic Evaluation of Nonadiabatic Coupling Terms at the MR-CI Level. II. Minima on the Crossing Seam: Formaldehyde and the Photodimerization of Ethylene. J. Chem. Phys. 2004, 120, 7330-7339.

(214) Lischka, H.; Dallos, M.; Szalay, P. G.; Yarkony, D. R.; Shepard, R. Analytic Evaluation of Nonadiabatic Coupling Terms at the MR-CI Level. I. Formalism. J. Chem. Phys. 2004, 120, $7322-7329$.

(215) Barbatti, M.; Lischka, H. Nonadiabatic Deactivation of 9H-Adenine: A Comprehensive Picture Based on Mixed Quantum-Classical Dynamics. J. Am. Chem. Soc. 2008, 130, 6831-6839. (216) Kuhlman, T. S.; Glover, W. J.; Mori, T.; Møller, K. B.; Martínez, T. J. Between Ethylene and Polyenes - the Non-Adiabatic Dynamics of cIs-Dienes. Faraday Discuss. 2012, 157, 193-193. (217) Mori, T.; Glover, W. J.; Schuurman, M. S.; Martinez, T. J. Role of Rydberg States in the Photochemical Dynamics of Ethylene. J. Phys. Chem. A 2012, 116, 2808-2818. 
(218) Liu, L.; Liu, J.; Martinez, T. J. Dynamical Correlation Effects on Photoisomerization: Ab Initio Multiple Spawning Dynamics with MS-CASPT2 for a Model trans-Protonated Schiff Base. J. Phys. Chem. B 2016, 120, 1940-1949.

(219) Tao, H. L.; Levine, B. G.; Martinez, T. J. Ab Initio Multiple Spawning Dynamics Using Multi-State Second-Order Perturbation Theory. J. Phys. Chem. A 2009, 113, 13656-13662.

(220) Coe, J. D.; Levine, B. G.; Martínez, T. J. Ab Initio Molecular Dynamics of Excited-State Intramolecular Proton Transfer Using Multireference Perturbation Theory. J. Phys. Chem. A 2007, $111,11302-11310$.

(221) Mai, S.; Marquetand, P.; González, L. Intersystem Crossing Pathways in the Noncanonical Nucleobase 2-Thiouracil: A Time-Dependent Picture. J. Phys. Chem. Lett. 2016, 7, 1978-1983.

(222) Park, J. W.; Shiozaki, T. Analytical Derivative Coupling for Multistate CASPT2 Theory. J. Chem. Theory Comput. 2017, 13, 2561-2570.

(223) Park, J. W.; Shiozaki, T. On-the-Fly CASPT2 Surface-Hopping Dynamics. J. Chem. Theory Comput. 2017, DOI:10.1021/acs.jctc.7b00559.

(224) Weber, W.; Thiel, W. Orthogonalization Corrections for Semiempirical Methods. Theor. Chem. Acc. 2000, 103, 495-506.

(225) Koslowski, A.; Beck, M. E.; Thiel, W. Implementation of a General Multireference Configuration Interaction Procedure with Analytic Gradients in a Semiempirical Context Using the Graphical Unitary Group Approach. J. Comput. Chem. 2003, 24, 714-726.

(226) Patchkovskii, S.; Koslowski, A.; Thiel, W. Generic Implementation of Semi-Analytical CI Gradients for NDDO-Type Methods. Theor. Chem. Acc. 2005, 114, 84-89.

(227) Granucci, G.; Toniolo, A. Molecular Gradients for Semiempirical CI Wavefunctions with Floating Occupation Molecular Orbitals. Chem. Phys. Lett. 2000, 325, 79-85.

(228) Toniolo, A.; Persico, M.; Pitea, D. An Ab Initio Study of Spectroscopy and Predissociation of ClO. J. Chem. Phys. 2000, 112, 2790-2797.

(229) Slavicek, P.; Martinez, T. J. Ab Initio Floating Occupation Molecular Orbital-Complete Active Space Configuration Interaction: An Efficient Approximation to CASSCF. J. Chem. Phys. 2010, 132, 234102.

(230) Bernardi, F.; Olivucci, M.; Robb, M. A. Simulation of MC-SCF Results on Covalent Organic Multi-Bond Reactions: Molecular Mechanics with Valence Bond (MM-VB). J. Am. Chem. Soc. 1992, 114, 1606-1616.

(231) Silva-Junior, M. R.; Thiel, W. Benchmark of Electronically Excited States for Semiempirical Methods: MNDO, AM1, PM3, OM1, OM2, OM3, INDO/S, and INDO/S2. J. Chem. Theory Comput. 2010, 6, 1546-1564.

(232) Dral, P. O.; von Lilienfeld, O. A.; Thiel, W. Machine Learning of Parameters for Accurate Semiempirical Quantum Chemical Calculations. J. Chem. Theory Comput. 2015, 11, 2120-2125. (233) Grimme, S.; Waletzke, M. A Combination of Kohn-Sham Density Functional Theory and Multi-Reference Configuration Interaction Methods. J. Chem. Phys. 1999, 111, 5645-5655.

(234) Lyskov, I.; Kleinschmidt, M.; Marian, C. M. Redesign of the DFT/MRCI Hamiltonian. $J$. Chem. Phys. 2016, 144, 034104.

(235) Filatov, M. Spin-Restricted Ensemble-Referenced Kohn-sham Method: Basic Principles and Application to Strongly Correlated Ground and Excited States of Molecules. WIREs: Comp. Mol. Sci. 2014, 5, 146-167.

(236) Fromager, E.; Knecht, S.; Jensen, H. J. A. Multi-Configuration Time-Dependent DensityFunctional Theory Based on Range Separation. J. Chem. Phys. 2013, 138, 084101. 
(237) Beck, E. V.; Stahlberg, E. A.; Burggraf, L. W.; Blaudeau, J.-P. A Graphical Unitary Group Approach-Based Hybrid Density Functional Theory Multireference Configuration Interaction Method. Chem. Phys. 2008, 349, 158-169.

(238) Li Manni, G.; Carlson, R. K.; Luo, S.; Ma, D.; Olsen, J.; Truhlar, D. G.; Gagliardi, L. Multiconfiguration Pair-Density Functional Theory. J. Chem. Theory Comput. 2014, 10, 36693680 .

(239) Gräfenstein, J.; Cremer, D. Development of a CAS-DFT Method Covering NonDynamical and Dynamical Electron Correlation in a Balanced Way. Mol. Phys. 2005, 103, 279308.

(240) Filatov, M.; Liu, F.; Martínez, T. J. Analytical Derivatives of The Individual State Energies In Ensemble Density Functional Theory Method. I. General Formalism. J. Chem. Phys. 2017, 147, 034113.

(241) Cremer, D. Density Functional Theory: Coverage of Dynamic and Non-Dynamic Electron Correlation Effects. Mol. Phys. 2001, 99, 1899-1940.

(242) Becke, A. D. Perspective: Fifty Years of Density-Functional Theory in Chemical Physics. J. Chem. Phys. 2014, 140, 18A301.

(243) Filatov, M.; Liu, F.; Kim, K. S.; Martínez, T. J. Self-Consistent Implementation of Ensemble Density Functional Theory Method for Multiple Strongly Correlated Electron Pairs. J. Chem. Phys. 2016, 145, 244104.

(244) Filatov, M.; Martínez, T. J.; Kim, K. S. Description of Ground and Excited Electronic States by Ensemble Density Functional Method with Extended Active Space. J. Chem. Phys. 2017, 147, 064104.

(245) Alam, M. M.; Knecht, S.; Fromager, E. Ghost-Interaction Correction in Ensemble DensityFunctional Theory for Excited States with and Without Range Separation. Phys. Rev. A 2016, 94, 012511.

(246) Gagliardi, L.; Truhlar, D. G.; Li Manni, G.; Carlson, R. K.; Hoyer, C. E.; Bao, J. L. Multiconfiguration Pair-Density Functional Theory: A New Way To Treat Strongly Correlated Systems. Acc. Chem. Res. 2017, 50, 66-73.

(247) Klein, S.; Bearpark, M. J.; Smith, B. R.; Robb, M. A.; Olivucci, M.; Bernardi, F. Mixed State 'On the Fly' Non-Adiabatic Dynamics: The Role of the Conical Intersection Topology. Chem. Phys. Lett. 1998, 292, 259-266.

(248) Nelson, T.; Fernandez-Alberti, S.; Roitberg, A. E.; Tretiak, S. Electronic Delocalization, Vibrational Dynamics, and Energy Transfer in Organic Chromophores. J. Phys. Chem. Lett. 2017, 8, 3020-3031.

(249) Tajti, A.; Szalay, P. G. Analytic Evaluation of the Nonadiabatic Coupling Vector between Excited States Using Equation-of-Motion Coupled-Cluster Theory. J. Chem. Phys. 2009, 131, 124104.

(250) Levine, B. G.; Ko, C.; Quenneville, J.; Martínez, T. J. Conical Intersections and Double Excitations in Time-Dependent Density Functional Theory. Mol. Phys. 2006, 104, 1039-1051.

(251) Gozem, S.; Melaccio, F.; Valentini, A.; Filatov, M.; Huix-Rotllant, M.; Ferré, N.; Frutos, L. M.; Angeli, C.; Krylov, A. I.; Granovsky, A. A., et al. Shape of Multireference, Equation-ofMotion Coupled-Cluster, and Density Functional Theory Potential Energy Surfaces at a Conical Intersection. J. Chem. Theory Comput. 2014, 10, 3074-3084.

(252) Tuna, D.; Lefrancois, D.; Wolański, Ł.; Gozem, S.; Schapiro, I.; Andruniów, T.; Dreuw, A.; Olivucci, M. Assessment of Approximate Coupled-Cluster and Algebraic-Diagrammatic- 
Construction Methods for Ground- and Excited-State Reaction Paths and the Conical-Intersection Seam of a Retinal-Chromophore Model. J. Chem. Theory Comput. 2015, 11, 5758-5781.

(253) Tapavicza, E.; Tavernelli, I.; Rothlisberger, U.; Filippi, C.; Casida, M. E. Mixed TimeDependent Density-Functional Theory/Classical Trajectory Surface Hopping Study of Oxirane Photochemistry. J. Chem. Phys. 2008, 129, 124108.

(254) Wormit, M.; Harbach, P. H. P.; Mewes, J. M.; Amarie, S.; Wachtveitl, J.; Dreuw, A. Excitation Energy Transfer and Carotenoid Radical Cation Formation in Light Harvesting Complexes - a Theoretical Perspective. Biochimica et Biophysica Acta (BBA) - Bioenergetics 2009, 1787, 738-746.

(255) Stojanović, L.; Bai, S.; Nagesh, J.; Izmaylov, A.; Crespo-Otero, R.; Lischka, H.; Barbatti, M. New Insights into the State Trapping of UV-Excited Thymine. Molecules 2016, 21, 1603.

(256) Casanova, D.; Slipchenko, L. V.; Krylov, A. I.; Head-Gordon, M. Double Spin-Flip Approach within Equation-of-Motion Coupled Cluster and Configuration Interaction Formalisms: Theory, Implementation, and Examples. J. Chem. Phys. 2009, 130, 044103.

(257) Krylov, A. I. Spin-Flip Configuration Interaction: An Electronic Structure Model That Is Both Variational and Size-Consistent. Chem. Phys. Lett. 2001, 350, 522-530.

(258) Lefrancois, D.; Wormit, M.; Dreuw, A. Adapting Algebraic Diagrammatic Construction Schemes for the Polarization Propagator to Problems with Multi-Reference Electronic Ground States Exploiting the Spin-Flip Ansatz. J. Chem. Phys. 2015, 143, 124107.

(259) Olsen, J.; Jørgensen, P., Time-Dependent Response Theory with Applications to SelfConsistent Field and Multiconfigurational Self-Consistent Field Wave Functions. In Modern Electronic Structure Theory Part II, Yarkony, D. R., Ed. World Scientific Publishing Company: 1995; pp 857-990.

(260) Sneskov, K.; Christiansen, O. Excited State Coupled Cluster Methods. WIREs: Comp. Mol. Sci. 2012, 2, 566-584.

(261) Hättig, C., Beyond Hartree-Fock: MP2 and Coupled-Cluster Methods for large systems. In Computational Nanoscience: Do It Yourself!, Grotendort, J.; Blügel, S.; Marx, D., Eds. Forschungszentrum Jülich: Jülich, 2006; pp 1-34.

(262) Hättig, C., Structure Optimizations for Excited States with Correlated Second-Order Methods: CC2 and ADC(2). In Adv. Quantum Chem., Jensen, H. J. A., Ed. Academic Press: 2005; Vol. Volume 50, pp 37-60.

(263) Plasser, F.; Crespo-Otero, R.; Pederzoli, M.; Pittner, J.; Lischka, H.; Barbatti, M. Surface Hopping Dynamics with Correlated Single-Reference Methods: $9 H-A d e n i n e$ as a Case Study. $J$. Chem. Theory Comput. 2014, 10, 1395-1405.

(264) Trofimov, A. B.; Schirmer, J. An Efficient Polarization Propagator Approach to Valence Electron Excitation Spectra. J. Phys. B: At., Mol. Opt. Phys. 1995, 28, 2299-2324.

(265) Trofimov, A. B.; Schirmer, J. Polarization Propagator Study of Electronic Excitation in Key Heterocyclic Molecules I. Pyrrole. Chem. Phys. 1997, 214, 153-170.

(266) Dreuw, A.; Wormit, M. The Algebraic Diagrammatic Construction Scheme for the Polarization Propagator for the Calculation of Excited States. WIREs: Comp. Mol. Sci. 2015, 5 , 82-95.

(267) Starcke, J. H.; Wormit, M.; Schirmer, J.; Dreuw, A. How Much Double Excitation Character Do the Lowest Excited States of Linear Polyenes Have? Chem. Phys. 2006, 329, 39-49. (268) Harbach, P. H. P.; Wormit, M.; Dreuw, A. The Third-Order Algebraic Diagrammatic Construction Method (ADC(3)) for the Polarization Propagator for Closed-Shell Molecules: Efficient Implementation and Benchmarking. J. Chem. Phys. 2014, 141, 064113. 
(269) Wenzel, J.; Wormit, M.; Dreuw, A. Calculating Core-Level Excitations and X-Ray Absorption Spectra of Medium-Sized Closed-Shell Molecules with the Algebraic-Diagrammatic Construction Scheme for the Polarization Propagator. J. Comput. Chem. 2014, 35, 1900-1915.

(270) Kochman, M. A.; Pola, M.; Dwayne Miller, R. J. Theoretical Study of the Photophysics of 8-Vinylguanine, an Isomorphic Fluorescent Analogue of Guanine. J. Phys. Chem. A 2016, 120, 6200-6215.

(271) Kochman, M. A.; Tajti, A.; Morrison, C. A.; Miller, R. J. D. Early Events in the Nonadiabatic Relaxation Dynamics of 4-( N , N -Dimethylamino)benzonitrile. J. Chem. Theory Comput. 2015, 11, 1118-1128.

(272) Szabla, R.; Gora, R. W.; Sponer, J. Ultrafast Excited-State Dynamics of Isocytosine. Phys. Chem. Chem. Phys. 2016, 18, 20208-20218.

(273) Prlj, A.; Curchod, B. F. E.; Corminboeuf, C. Excited State Dynamics of Thiophene and Bithiophene: New Insights into Theoretically Challenging Systems. Phys. Chem. Chem. Phys. 2015, 17, 14719-30.

(274) Chaiwongwattana, S.; Sapunar, M.; Ponzi, A.; Decleva, P.; Došlić, N. Exploration of Excited State Deactivation Pathways of Adenine Monohydrates. J. Phys. Chem. A 2015, 119, 10637-10644.

(275) Szabla, R.; Góra, R. W.; Janicki, M.; Šponer, J. Photorelaxation of Imidazole and Adenine via Electron-Driven Proton Transfer along $\mathrm{H}_{2} \mathrm{O}$ Wires. Faraday Discuss. Chem. Soc. 2016, 195, 237-251.

(276) Barbatti, M. Photorelaxation Induced by Water-Chromophore Electron Transfer. J. Am. Chem. Soc. 2014, 136, 10246-10249.

(277) Szabla, R.; Šponer, J.; Góra, R. W. Electron-Driven Proton Transfer Along $\mathrm{H}_{2} \mathrm{O}$ Wires Enables Photorelaxation of $\pi \sigma^{*}$ States in Chromophore-Water Clusters. J. Phys. Chem. Lett. 2015, 6, 1467-1471.

(278) Thisuwan, J.; Chaiwongwattana, S.; Sapunar, M.; Sagarik, K.; Došlić, N. Photochemical Deactivation Pathways of Microsolvated Hydroxylamine. J. Photochem. Photobiol. A 2016, 328, $10-15$.

(279) Dommett, M.; Crespo-Otero, R. Excited State Proton Transfer in 2'-Hydroxychalcone Derivatives. Phys. Chem. Chem. Phys. 2017, 19, 2409-2416.

(280) Crespo-Otero, R.; Kungwan, N.; Barbatti, M. Stepwise Double Excited-State Proton Transfer Is Not Possible in 7-Azaindole Dimer. Chem. Sci. 2015, 6, 5762-5767.

(281) Prlj, A.; Sandoval-Salinas, M. E.; Casanova, D.; Jacquemin, D.; Corminboeuf, C. LowLying $\pi \pi^{*}$ States of Heteroaromatic Molecules: A Challenge for Excited State Methods. J. Chem. Theory Comput. 2016, 12, 2652-2660.

(282) Kjønstad, E. F.; Myhre, R. H.; Martínez, T. J.; Koch, H. Crossing Conditions in Coupled Cluster Theory. J. Chem. Phys. 2017, 147, 164105.

(283) Lefrancois, D.; Tuna, D.; Martínez, T. J.; Dreuw, A. The Spin-Flip Variant of the Algebraic-Diagrammatic Construction Yields the Correct Topology of $\mathrm{S}_{1} / \mathrm{S}_{0}$ Conical Intersections. J. Chem. Theory Comput. 2017, 13, 4436-4441.

(284) Janssen, C. L.; Nielsen, I. M. B. New Diagnostics for Coupled-Cluster and Møller-Plesset Perturbation Theory. Chem. Phys. Lett. 1998, 290, 423-430.

(285) Nielsen, I. M. B.; Janssen, C. L. Double-Substitution-Based Diagnostics for CoupledCluster and Møller-plesset Perturbation Theory. Chem. Phys. Lett. 1999, 310, 568-576. 
(286) Sapunar, M.; Ponzi, A.; Chaiwongwattana, S.; Malis, M.; Prlj, A.; Decleva, P.; Došlić, N. Timescales of N-H Bond Dissociation in Pyrrole: A Nonadiabatic Dynamics Study. Phys. Chem. Chem. Phys. 2015, 17, 19012-19020.

(287) Parker, S. M.; Roy, S.; Furche, F. Unphysical Divergences in Response Theory. J. Chem. Phys. 2016, 145, 134105.

(288) Dreuw, A.; Head-Gordon, M. Single-Reference ab Initio Methods for the Calculation of Excited States of Large Molecules. Chem. Rev. 2005, 105, 4009-4037.

(289) Casida, M. E.; Huix-Rotllant, M. Progress in Time-Dependent Density-Functional Theory. Annu. Rev. Phys. Chem. 2012, 63, 287-323.

(290) Li, X.; Smith, S. M.; Markevitch, A. N.; Romanov, D. A.; Levis, R. J.; Schlegel, H. B. A Time-Dependent Hartree-Fock Approach for Studying the Electronic Optical Response of Molecules in Intense Fields. Phys. Chem. Chem. Phys. 2005, 7, 233-239.

(291) Chen, G.; Mukamel, S.; Beljonne, D.; Brédas, J. L. The Coupled Electronic Oscillators vs the Sum-over-States Pictures for the Optical Response of Octatetraene. J. Chem. Phys. 1996, 104, 5406-5414.

(292) Tretiak, S.; Mukamel, S. Density Matrix Analysis and Simulation of Electronic Excitations in Conjugated and Aggregated Molecules. Chem. Rev. 2002, 102, 3171-3212.

(293) Nelson, T.; Fernandez-Alberti, S.; Chernyak, V.; Roitberg, A. E.; Tretiak, S. Nonadiabatic Excited-State Molecular Dynamics Modeling of Photoinduced Dynamics in Conjugated Molecules. J. Phys. Chem. B 2011, 115, 5402-5414.

(294) Nelson, T.; Fernandez-Alberti, S.; Chernyak, V.; Roitberg, A. E.; Tretiak, S. Nonadiabatic Excited-State Molecular Dynamics: Numerical Tests of Convergence and Parameters. J. Chem. Phys. 2012, 136, 054108-12.

(295) Casida, M., Time-Dependent Density Functional Response Theory for Molecules. In Recent advances in density functional methods, Part I, Chong, D., Ed. World Scientific: Singapore, 1995; pp 155-192.

(296) Gonze, X.; Scheffler, M. Exchange and Correlation Kernels at the Resonance Frequency: Implications for Excitation Energies in Density-Functional Theory. Phys. Rev. Lett. 1999, 82, 4416-4419.

(297) Marques, M. A. L.; Gross, E. K. U. Time-Dependent Density Functional Theory. Annu. Rev. Phys. Chem. 2004, 55, 427-455.

(298) Hirata, S.; Head-Gordon, M. Time-Dependent Density Functional Theory within the Tamm-dancoff Approximation. Chem. Phys. Lett. 1999, 314, 291-299.

(299) Curchod, B. F. E.; Rothlisberger, U.; Tavernelli, I. Trajectory-Based Nonadiabatic Dynamics with Time-Dependent Density Functional Theory. ChemPhysChem 2013, 14, 13141340.

(300) Shao, Y.; Head-Gordon, M.; Krylov, A. I. The Spin-flip Approach within Time-Dependent Density Functional Theory: Theory and Applications to Diradicals. J. Chem. Phys. 2003, 118, 4807-4818.

(301) Xu, X.; Yang, K. R.; Truhlar, D. G. Testing Noncollinear Spin-Flip, Collinear Spin-Flip, and Conventional Time-Dependent Density Functional Theory for Predicting Electronic Excitation Energies of Closed-Shell Atoms. J. Chem. Theory Comput. 2014, 10, 2070-2084.

(302) Harabuchi, Y.; Keipert, K.; Zahariev, F.; Taketsugu, T.; Gordon, M. S. Dynamics Simulations with Spin-Flip Time-Dependent Density Functional Theory: Photoisomerization and Photocyclization Mechanisms of cis-Stilbene in $\pi \pi^{*}$ States. J. Phys. Chem. A 2014, 118, 1198711998. 
(303) Peach, M. J. G.; Helgaker, T.; Salek, P.; Keal, T. W.; Lutnaes, O. B.; Tozer, D. J.; Handy, N. C. Assessment of a Coulomb-Attenuated Exchange-Correlation Energy Functional. Phys. Chem. Chem. Phys. 2005, 8, 558-562.

(304) Dreuw, A.; Weisman, J. L.; Head-Gordon, M. Long-Range Charge-Transfer Excited States in Time-Dependent Density Functional Theory Require Non-Local Exchange. J. Chem. Phys. 2003, 119, 2943-2946.

(305) Hellgren, M.; Gross, E. K. U. Discontinuities of the Exchange-Correlation Kernel and Charge-Transfer Excitations in Time-Dependent Density-Functional Theory. Phys. Rev. A 2012, $85,022514$.

(306) Rivalta, I.; Nenov, A.; Cerullo, G.; Mukamel, S.; Garavelli, M. Ab Initio Simulations of Two-Dimensional Electronic Spectra: The SOS//QM/MM Approach. Int. J. Quantum Chem. 2014, $114,85-93$.

(307) van der Vegte, C. P.; Dijkstra, A. G.; Knoester, J.; Jansen, T. L. C. Calculating TwoDimensional Spectra with the Mixed Quantum-Classical Ehrenfest Method. J. Phys. Chem. A 2013, 117, 5970-5980.

(308) Yanai, T.; Tew, D. P.; Handy, N. C. A New Hybrid Exchange-Correlation Functional Using the Coulomb-Attenuating Method (CAM-B3LYP). Chem. Phys. Lett. 2004, 393, 51-57.

(309) Chai, J.-D.; Head-Gordon, M. Systematic Optimization of Long-Range Corrected Hybrid Density Functionals. J. Chem. Phys. 2008, 128, 084106-15.

(310) Casida, M. E.; Jamorski, C.; Casida, K. C.; Salahub, D. R. Molecular Excitation Energies to High-Lying Bound States from Time-Dependent density-Functional response theory: Characterization and Correction of the time-Dependent local density approximation Ionization Threshold. J. Chem. Phys. 1998, 108, 4439-4449.

(311) Stojanović, L.; Alyoubi, A. O.; Aziz, S. G.; Hilal, R. H.; Barbatti, M. UV Excitations of Halons. J. Chem. Phys. 2016, 145, 184306.

(312) Duffy, P.; Chong, D. P.; Casida, M. E.; Salahub, D. R. Assessment of Kohn-Sham DensityFunctional Orbitals as Approximate Dyson Orbitals for the Calculation of Electron-MomentumSpectroscopy Scattering Cross Sections. Phys. Rev. A 1994, 50, 4707.

(313) Hirata, S.; Zhan, C.-G.; Aprà, E.; Windus, T. L.; Dixon, D. A. A New, Self-Contained Asymptotic Correction Scheme To Exchange-Correlation Potentials for Time-Dependent Density Functional Theory. J. Phys. Chem. A 2003, 107, 10154-10158.

(314) Niehaus, T. A.; Suhai, S.; Della Sala, F.; Lugli, P.; Elstner, M.; Seifert, G.; Frauenheim, T. Tight-Binding Approach to Time-Dependent Density-Functional Response Theory. Phys. Rev. B 2001, 63, 085108.

(315) Elstner, M.; Porezag, D.; Jungnickel, G.; Elsner, J.; Haugk, M.; Frauenheim, T.; Suhai, S.; Seifert, G. Self-Consistent-Charge Density-Functional Tight-Binding Method for Simulations of Complex Materials Properties. Phys. Rev. B 1998, 58, 7260-7268.

(316) Gaus, M.; Cui, Q.; Elstner, M. Density Functional Tight Binding: Application to Organic and Biological Molecules. Wiley Interdisciplinary Reviews: Computational Molecular Science 2014, 4, 49-61.

(317) Elstner, M.; Seifert, G. Density Functional Tight Binding. Philos. Trans. R. Soc. London, A 2014, 372, 20120483.

(318) Domínguez, A.; Aradi, B.; Frauenheim, T.; Lutsker, V.; Niehaus, T. A. Extensions of the Time-Dependent Density Functional Based Tight-Binding Approach. J. Chem. Theory Comput. 2013, 9, 4901-4914. 
(319) Kranz, J. J.; Elstner, M.; Aradi, B.; Frauenheim, T.; Lutsker, V.; Garcia, A. D.; Niehaus, T. A. Time-Dependent Extension of the Long-Range Corrected Density Functional Based TightBinding Method. J. Chem. Theory Comput. 2017, 13, 1737-1747.

(320) Lutsker, V.; Aradi, B.; Niehaus, T. A. Implementation and Benchmark of a Long-Range Corrected Functional in the Density Functional Based Tight-Binding Method. J. Chem. Phys. 2015, 143, 184107.

(321) Mitrić, R.; Werner, U.; Wohlgemuth, M.; Seifert, G.; Bonačić-Koutecký, V. Nonadiabatic Dynamics within Time-Dependent Density Functional Tight Binding Method. J. Phys. Chem. A 2009, 113, 12700-12705.

(322) Stojanović, L.; Aziz, S. G.; Hilal, R. H.; Plasser, F.; Niehaus, T. A.; Barbatti, M. Nonadiabatic Dynamics of Cycloparaphenylenes with TD-DFTB Surface Hopping. J. Chem. Theory Comput. 2017, 13, 5846-5860.

(323) Kungwan, N.; Plasser, F.; Aquino, A. J. A.; Barbatti, M.; Wolschann, P.; Lischka, H. The Effect of Hydrogen Bonding on the Excited-State Proton Transfer in 2-(2'Hydroxyphenyl)Benzothiazole: A TDDFT Molecular Dynamics Study. Phys. Chem. Chem. Phys. 2012, 14, 9016-9025.

(324) Fazzi, D.; Barbatti, M.; Thiel, W. Unveiling the Role of Hot Charge-Transfer States in Molecular Aggregates via Nonadiabatic Dynamics. J. Am. Chem. Soc. 2016, 138, 4502-4511.

(325) Crespo-Otero, R.; Barbatti, M.; Yu, H.; Evans, N. L.; Ullrich, S. Ultrafast Dynamics of UV-Excited Imidazole. ChemPhys Chem 2011, 12, 3365-3375.

(326) Marchetti, B.; Karsili, T. N. V. Theoretical Insights into the Photo-Protective Mechanisms of Natural Biological Sunscreens: Building Blocks of Eumelanin and Pheomelanin. Phys. Chem. Chem. Phys. 2016, 18, 3644-3658.

(327) Cisneros, C.; Thompson, T.; Baluyot, N.; Smith, A. C.; Tapavicza, E. The Role of Tachysterol in Vitamin D Photosynthesis - a Non-Adiabatic Molecular Dynamics Study. Phys. Chem. Chem. Phys. 2017, 19, 5763-5777.

(328) Mališ, M.; Došlić, N. Nonradiative Relaxation Mechanisms of UV Excited Phenylalanine Residues: A Comparative Computational Study. Molecules 2017, 22, 493.

(329) Tuna, D.; Došlić, N.; Mališ, M.; Sobolewski, A. L.; Domcke, W. Mechanisms of Photostability in Kynurenines: A Joint Electronic-Structure and Dynamics Study. J. Phys. Chem. B 2015, 119, 2112-2124.

(330) Jankowska, J.; Barbatti, M.; Sadlej, J.; Sobolewski, A. L. Tailoring the Schiff Base Photoswitching - a Non-adiabatic Molecular Dynamics Study of Substituent Effect on Excited State Proton Transfer. Phys. Chem. Chem. Phys. 2017, 19, 5318-5325.

(331) Novak, J.; Mališ, M.; Prlj, A.; Ljubić, I.; Kühn, O.; Došlić, N. Photoinduced Dynamics of Formic Acid Monomers and Dimers: The Role of the Double Hydrogen Bond. J. Phys. Chem. A 2012, 116, 11467-11475.

(332) Pereira Rodrigues, G.; Ventura, E.; do Monte, S. A.; Barbatti, M. Photochemical Deactivation Process of HCFC-133a $\left(\mathrm{C}_{2} \mathrm{H}_{2} \mathrm{~F}_{3} \mathrm{Cl}\right)$ : A Nonadiabatic Dynamics Study. J. Phys. Chem. A 2014, 118, 12041-12049.

(333) Crespo-Otero, R.; Barbatti, M. Cr(CO) 6 Photochemistry: Semi-Classical Study of UV Absorption Spectral Intensities and Dynamics of Photodissociation. J. Chem. Phys. 2011, 134, 164305.

(334) Tavernelli, I.; Curchod, B. F. E.; Rothlisberger, U. Nonadiabatic Molecular Dynamics with Solvent Effects: A LR-TDDFT QM/MM Study of Ruthenium (II) Tris (Bipyridine) in Water. Chem. Phys. 2011, 391, 101-109. 
(335) Capano, G.; Penfold, T. J.; Chergui, M.; Tavernelli, I. Photophysics of a Copper Phenanthroline Elucidated by Trajectory and Wavepacket-Based Quantum Dynamics: A Synergetic Approach. Phys. Chem. Chem. Phys. 2017, DOI:10.1039/C7CP00436B.

(336) Muuronen, M.; Parker, S. M.; Berardo, E.; Le, A.; Zwijnenburg, M. A.; Furche, F. Mechanism of Photocatalytic Water Oxidation on small $\mathrm{TiO}_{2}$ Nanoparticles. Chem. Sci. 2017, 8, 2179-2183.

(337) Wiebeler, C.; Plasser, F.; Hedley, G. J.; Ruseckas, A.; Samuel, I. D. W.; Schumacher, S. Ultrafast Electronic Energy Transfer in an Orthogonal Molecular Dyad. J. Phys. Chem. Lett. 2017, 8, 1086-1092.

(338) Yabana, K.; Bertsch, G. F. Time-Dependent Local-Density Approximation in Real Time. Phys. Rev. B 1996, 54, 4484-4487.

(339) Rubio, A.; Alonso, J. A.; Blase, X.; Louie, S. G. Theoretical Models for the Optical Properties of Clusters and Nanostructures. Int. J. Mod. Phys. B 1997, 11, 2727-2776.

(340) Andrade, X.; Castro, A.; Zueco, D.; Alonso, J. L.; Echenique, P.; Falceto, F.; Rubio, Á. Modified Ehrenfest Formalism for Efficient Large-Scale ab initio Molecular Dynamics. J. Chem. Theory Comput. 2009, 5, 728-742.

(341) Sugino, O.; Miyamoto, Y. Density-Functional Approach to Electron Dynamics: Stable Simulation under a Self-Consistent Field. Phys. Rev. B 1999, 59, 2579-2586.

(342) Ojanperä, A.; Havu, V.; Lehtovaara, L.; Puska, M. Nonadiabatic Ehrenfest Molecular Dynamics within the Projector Augmented-Wave Method. J. Chem. Phys. 2012, 136, 144103.

(343) Li, X. S.; Tully, J. C.; Schlegel, H. B.; Frisch, M. J. Ab Initio Ehrenfest Dynamics. J. Chem. Phys. 2005, 123, 084106-.

(344) Akama, T.; Nakai, H. Short-Time Fourier Transform Analysis of Real-Time TimeDependent Hartree-fock and Time-Dependent Density Functional Theory Calculations with Gaussian Basis Functions. J. Chem. Phys. 2010, 132, 054104.

(345) Lopez-Lozano, X.; Barron, H.; Mottet, C.; Weissker, H.-C. Aspect-Ratio- and SizeDependent Emergence of the Surface-Plasmon Resonance in Gold Nanorods - an Ab Initio TDDFT Study. Phys. Chem. Chem. Phys. 2014, 16, 1820-1823.

(346) Lopata, K.; Govind, N. Modeling Fast Electron Dynamics with Real-Time TimeDependent Density Functional Theory: Application to Small Molecules and Chromophores. $J$. Chem. Theory Comput. 2011, 7, 1344-1355.

(347) Mendive-Tapia, D.; Vacher, M.; Bearpark, M. J.; Robb, M. A. Coupled electron-nuclear dynamics: Charge migration and charge transfer initiated near a conical intersection. J. Chem. Phys. 2013, 139, 044110.

(348) Lei, Y.; Yuan, S.; Dou, Y.; Wang, Y.; Wen, Z. Detailed Dynamics of the Nonradiative Deactivation of Adenine: A Semiclassical Dynamics Study. J. Phys. Chem. A 2008, 112, 84978504.

(349) Craig, C. F.; Duncan, W. R.; Prezhdo, O. V. Trajectory Surface Hopping in the TimeDependent Kohn-Sham Approach for Electron-Nuclear Dynamics. Phys. Rev. Lett. 2005, 95, 163001.

(350) Fischer, S. A.; Habenicht, B. F.; Madrid, A. B.; Duncan, W. R.; Prezhdo, O. V. Regarding the Validity of the Time-Dependent Kohn-sham Approach for Electron-Nuclear Dynamics via Trajectory Surface Hopping. J. Chem. Phys. 2011, 134, 024102.

(351) Akimov, A. V.; Prezhdo, O. V. The PYXAID Program for Non-Adiabatic Molecular Dynamics in Condensed Matter Systems. J. Chem. Theory Comput. 2013, 9, 4959-4972. 
(352) Senanayake, R. D.; Akimov, A. V.; Aikens, C. M. Theoretical Investigation of Electron and Nuclear Dynamics in the $\left[\mathrm{Au}_{25}(\mathrm{SH})_{18}\right]^{-1}$ Thiolate-Protected Gold Nanocluster. J. Phys. Chem. C 2017, 121, 10653-10662.

(353) Long, R.; Casanova, D.; Fang, W.-H.; Prezhdo, O. V. Donor-Acceptor Interaction Determines the Mechanism of Photoinduced Electron Injection from Graphene Quantum Dots into TiO2: $\pi$-Stacking Supersedes Covalent Bonding. J. Am. Chem. Soc. 2017, 139, 2619-2629.

(354) Gao, X.; Peng, Q.; Niu, Y.; Wang, D.; Shuai, Z. Theoretical Insight into the Aggregation Induced Emission Phenomena of Diphenyldibenzofulvene: A Nonadiabatic Molecular Dynamics Study. Phys. Chem. Chem. Phys. 2012, 14, 14207-14216.

(355) Pal, S.; Trivedi, D. J.; Akimov, A. V.; Aradi, B.; Frauenheim, T.; Prezhdo, O. V. Nonadiabatic Molecular Dynamics for Thousand Atom Systems: A Tight-Binding Approach toward PYXAID. J. Chem. Theory Comput. 2016, 12, 1436-1448.

(356) Maitra, N. T. On Correlated Electron-Nuclear Dynamics Using Time-Dependent Density Functional Theory. J. Chem. Phys. 2006, 125, 014110.

(357) Doltsinis, N. L., Nonadiabatic Dynamics: Mean-field and Surface Hopping. In Quantum Simulations of Complex Many-Body Systems: From Theory to Algorithms, Grotendorst, J.; Marx, D.; Muramatsu, A., Eds. John von Neumann Institute for Computing: Julich, 2002; Vol. 10, pp 377-397.

(358) Doltsinis, N. L.; Marx, D. Nonadiabatic Car-Parrinello Molecular Dynamics. Phys. Rev. Lett. 2002, 88, 166402.

(359) Car, R.; Parrinello, M. Unified Approach for Molecular Dynamics and Density-Functional Theory. Phys. Rev. Lett. 1985, 55, 2471-2474.

(360) Chernyak, V.; Mukamel, S. Density-Matrix Representation of Nonadiabatic Couplings in Time-Dependent Density Functional (TDDFT) Theories. J. Chem. Phys. 2000, 112, 3572-3579.

(361) Send, R.; Furche, F. First-Order Nonadiabatic Couplings from Time-Dependent Hybrid Density Functional Response Theory: Consistent Formalism, Implementation, and Performance. J. Chem. Phys. 2010, 132, 044107.

(362) Tavernelli, I.; Curchod, B. F. E.; Laktionov, A.; Rothlisberger, U. Nonadiabatic Coupling Vectors for Excited States within Time-Dependent Density Functional Theory in the Tammdancoff Approximation and Beyond. J. Chem. Phys. 2010, 133, 194104.

(363) Tavernelli, I.; Curchod, B. F. E.; Rothlisberger, U. On Nonadiabatic Coupling Vectors in Time-Dependent Density Functional Theory. J. Chem. Phys. 2009, 131, 196101.

(364) Baer, R. Non-Adiabatic Couplings by Time-Dependent Density Functional Theory. Chem. Phys. Lett. 2002, 364, 75-79.

(365) Hu, C.; Hirai, H.; Sugino, O. Nonadiabatic Couplings from Time-Dependent Density Functional Theory: Formulation in the Casida Formalism and Practical Scheme within Modified Linear Response. J. Chem. Phys. 2007, 127, 064103.

(366) Hu, C.; Sugino, O.; Tateyama, Y. All-Electron Calculation of Nonadiabatic Couplings from Time-Dependent Density Functional Theory: Probing with the Hartree-fock Exact Exchange. J. Chem. Phys. 2009, 131, 114101.

(367) Hu, C.; Sugino, O.; Watanabe, K. Performance of Tamm-Dancoff Approximation on Nonadiabatic Couplings by Time-Dependent Density Functional Theory. J. Chem. Phys. 2014, $140,054106$.

(368) Ou, Q.; Bellchambers, G. D.; Furche, F.; Subotnik, J. E. First-Order Derivative Couplings Between Excited States from Adiabatic TDDFT Response Theory. J. Chem. Phys. 2015, 142, 064114. 
(369) Barbatti, M.; Pittner, J.; Pederzoli, M.; Werner, U.; Mitrić, R.; Bonačić-Koutecký, V.; Lischka, H. Non-Adiabatic Dynamics of Pyrrole: Dependence of Deactivation Mechanisms on the Excitation Energy. Chem. Phys. 2010, 375, 26-34.

(370) Pittner, J.; Lischka, H.; Barbatti, M. Optimization of Mixed Quantum-Classical Dynamics: Time-Derivative Coupling Terms and Selected Couplings. Chem. Phys. 2009, 356, 147-152.

(371) Tapavicza, E.; Tavernelli, I.; Rothlisberger, U. Trajectory Surface Hopping within Linear Response Time-Dependent Density-Functional Theory. Phys. Rev. Lett. 2007, 98, 023001-4.

(372) Werner, U.; Mitrić, R.; Suzuki, T.; Bonačić-Koutecký, V. Nonadiabatic Dynamics within the Time Dependent Density Functional Theory: Ultrafast Photodynamics in Pyrazine. Chem. Phys. 2008, 349, 319-324.

(373) Gao, X.; Bai, S.; Fazzi, D.; Niehaus, T.; Barbatti, M.; Thiel, W. Evaluation of Spin-Orbit Couplings with Linear-Response Time-Dependent Density Functional Methods. J. Chem. Theory Comput. 2017, 13, 515-524.

(374) Mitric, R.; Bonacic-Koutecky, V.; Pittner, J.; Lischka, H. Ab Initio Nonadiabatic Dynamics Study of Ultrafast Radiationless Decay over Conical Intersections Illustrated on the $\mathrm{Na}_{3} \mathrm{~F} \mathrm{Cluster}$. J. Chem. Phys. 2006, 125, 024303.

(375) Cimiraglia, R.; Persico, M.; Tomasi, J. The Evaluation of Nonadiabatic Matrix Elements. A Comparison of Different Approximations Applied to LiH X-a ${ }^{1} \Sigma^{+}$. Chem. Phys. 1980, 53, 357363.

(376) Martínez, T. J. Ab Initio Molecular Dynamics around a Conical Intersection: $\mathrm{Li}(2 \mathrm{p})+\mathrm{H}_{2}$. Chem. Phys. Lett. 1997, 272, 139-147.

(377) Groenhof, G.; Bouxin-Cademartory, M.; Hess, B.; deVisser, S. P.; Berendsen, H. J. C.; Olivucci, M.; Mark, A. E.; Robb, M. A. Photoactivation of the Photoactive Yellow Protein: Why Photon Absorption Triggers a Trans-to-Cis Isomerization of the Chromophore in the Protein. $J$. Am. Chem. Soc. 2004, 126, 4228-4233.

(378) Weingart, O.; Schapiro, I.; Buss, V. Photochemistry of Visual Pigment Chromophore Models by Ab Initio Molecular Dynamics. J. Phys. Chem. B 2007, 111, 3782-3788.

(379) Mitrić, R.; Werner, U.; Bonačić-Koutecký, V. Nonadiabatic Dynamics and Simulation of Time Resolved Photoelectron Spectra within Time-Dependent Density Functional Theory: Ultrafast Photoswitching in Benzylideneaniline. J. Chem. Phys. 2008, 129, 164118.

(380) Löwdin, P.-O. Quantum Theory of Many-Particle Systems. I. Physical Interpretations by Means of Density Matrices, Natural Spin-Orbitals, and Convergence Problems in the Method of Configurational Interaction. Phys. Rev. 1955, 97, 1474.

(381) Plasser, F.; Ruckenbauer, M.; Mai, S.; Oppel, M.; Marquetand, P.; González, L. Efficient and Flexible Computation of Many-Electron Wave Function Overlaps. J. Chem. Theory Comput. 2016, 12, 1207-1219.

(382) Thorsteinsson, T.; Cooper, D. L. Exact Transformations of CI Spaces, VB Representations of CASSCF Wavefunctions and the Optimization of VB Wavefunctions. Theor. Chim. Acta 1996, 94, 233-245.

(383) Malmqvist, P. A. Calculation of Transition Density Matrices by Nonunitary Orbital Transformations. Int. J. Quantum Chem. 1986, 30, 479-494.

(384) Ryabinkin, I. G.; Nagesh, J.; Izmaylov, A. F. Fast Numerical Evaluation of TimeDerivative Nonadiabatic Couplings for Mixed Quantum-Classical Methods. J. Phys. Chem. Lett. 2015, 6, 4200-4203.

(385) Atkins, A. J.; González, L. Trajectory Surface-Hopping Dynamics Including Intersystem Crossing in $\left[\mathrm{Ru}(\mathrm{bpy})_{3}\right]^{2+}$. J. Phys. Chem. Lett. 2017, 8, 3840-3845. 
(386) Dinkelbach, F.; Kleinschmidt, M.; Marian, C. M. Assessment of Interstate Spin-Orbit Couplings from Linear Response Amplitudes. J. Chem. Theory Comput. 2017, 13, 749-766.

(387) Mitrić, R.; Petersen, J.; Wohlgemuth, M.; Werner, U.; Bonačić-Koutecký, V.; Wöste, L.; Jortner, J. Time-Resolved Femtosecond Photoelectron Spectroscopy by Field-Induced Surface Hopping. J. Phys. Chem. A 2011, 115, 3755-3765.

(388) Tavernelli, I.; Tapavicza, E.; Rothlisberger, U. Nonadiabatic Coupling Vectors within Linear Response Time-Dependent Density Functional Theory. J. Chem. Phys. 2009, 130, 124107. (389) Humeniuk, A.; Wohlgemuth, M.; Suzuki, T.; Mitrić, R. Time-Resolved Photoelectron Imaging Spectra from Non-Adiabatic Molecular Dynamics Simulations. J. Chem. Phys. 2013, 139, 134104.

(390) Arbelo-González, W.; Crespo-Otero, R.; Barbatti, M. Steady and Time-Resolved Photoelectron Spectra Based on Nuclear Ensembles. J. Chem. Theory Comput. 2016, 12, 50375049.

(391) Barbatti, M.; Crespo-Otero, R., Surface Hopping Dynamics with DFT Excited States. In Density-Functional Methods for Excited States, Ferré, N.; Filatov, M.; Huix-Rotllant, M., Eds. Springer International Publishing: Cham, 2016; pp 415-444.

(392) Bergsma, J. P.; Berens, P. H.; Wilson, K. R.; Fredkin, D. R.; Heller, E. J. Electronic-Spectra from Molecular-Dynamics - a Simple Approach. J. Phys. Chem. 1984, 88, 612-619.

(393) Polli, D.; Altoe, P.; Weingart, O.; Spillane, K. M.; Manzoni, C.; Brida, D.; Tomasello, G.; Orlandi, G.; Kukura, P.; Mathies, R. A., et al. Conical Intersection Dynamics of the Primary Photoisomerization Event in Vision. Nature 2010, 467, 440-443.

(394) Ruckenbauer, M.; Mai, S.; Marquetand, P.; González, L. Photoelectron Spectra of 2Thiouracil, 4-Thiouracil, and 2,4-Dithiouracil. J. Chem. Phys. 2016, 144, 074303.

(395) Hudock, H. R.; Martínez, T. J. Excited-State Dynamics of Cytosine Reveal Multiple Intrinsic Subpicosecond Pathways. ChemPhysChem 2008, 9, 2486-2490.

(396) Thompson, A. L.; Martinez, T. J. Time-Resolved Photoelectron Spectroscopy from First Principles: Excited State Dynamics of Benzene. Faraday Discuss. 2011, 150, 293-311.

(397) Hudock, H. R.; Levine, B. G.; Thompson, A. L.; Satzger, H.; Townsend, D.; Gador, N.; Ullrich, S.; Stolow, A.; Martínez, T. J. Ab Initio Molecular Dynamics and Time-Resolved Photoelectron Spectroscopy of Electronically Excited Uracil and Thymine. J. Phys. Chem. A 2007, $111,8500-8508$.

(398) McFarland, B. K.; Farrell, J. P.; Miyabe, S.; Tarantelli, F.; Aguilar, A.; Berrah, N.; Bostedt, C.; Bozek, J. D.; Bucksbaum, P. H.; Castagna, J. C., et al. Ultrafast X-Ray Auger Probing of Photoexcited Molecular Dynamics. Nat Commun 2014, 5, 4235.

(399) Fuji, T.; Suzuki, Y.-I.; Horio, T.; Suzuki, T.; Mitric, R.; Werner, U.; Bonacic-Koutecky, V. Ultrafast Photodynamics of Furan. J. Chem. Phys. 2010, 133, 234303-9.

(400) Stanzel, J.; Neeb, M.; Eberhardt, W.; Lisinetskaya, P. G.; Petersen, J.; Mitrić, R. Switching from Molecular to Bulklike Dynamics in Electronic Relaxation of a Small Gold Cluster. Phys. Rev. A 2012, 85, 013201.

(401) Huix-Rotllant, M.; Tamura, H.; Burghardt, I. Concurrent Effects of Delocalization and Internal Conversion Tune Charge Separation at Regioregular Polythiophene-Fullerene Heterojunctions. J. Phys. Chem. Lett. 2015, 6, 1702-1708.

(402) Wolf, T. J. A.; Kuhlman, T. S.; Schalk, O.; Martinez, T. J.; Moller, K. B.; Stolow, A.; Unterreiner, A. N. Hexamethylcyclopentadiene: Time-Resolved Photoelectron Spectroscopy and Ab Initio Multiple Spawning Simulations. Phys. Chem. Chem. Phys. 2014, 16, 11770-11779. 
(403) Tomasello, G.; Humeniuk, A.; Mitrić, R. Exploring Ultrafast Dynamics of Pyrazine by Time-Resolved Photoelectron Imaging. J. Phys. Chem. A 2014, 118, 8437-8445.

(404) Schinke, R., Photodissociation Dynamics: Spectroscopy and Fragmentation of Small Polyatomic Molecules. Cambridge University Press: Cambridge, 1995.

(405) Case, W. B. Wigner Functions and Weyl Transforms for Pedestrians. Am. J. Phys. 2008, 76, 937-946.

(406) Crespo-Otero, R.; Barbatti, M. Spectrum Simulation and Decomposition with Nuclear Ensemble: Formal Derivation and Application to Benzene, Furan and 2-Phenylfuran. Theor. Chem. Acc. 2012, 131, 1237.

(407) Li, S. L.; Truhlar, D. G. Franck-Condon Models for Simulating the Band Shape of Electronic Absorption Spectra. J. Chem. Theory Comput. 2017, 13, 2823-2830.

(408) Tannor, D. J.; Heller, E. J. Polyatomic Raman Scattering for General Harmonic Potentials. J. Chem. Phys. 1982, 77, 202-218.

(409) Kubo, R. A Stochastic Theory of Line Shape. Adv. Chem. Phys. 1969, 15, 101-127.

(410) Ben-Nun, M.; Martínez, T. J. Electronic Absorption and Resonance Raman Spectroscopy from Ab Initio Quantum Molecular Dynamics. J. Phys. Chem. A 1999, 103, 10517-10527.

(411) Petit, A. S.; Subotnik, J. E. Appraisal of Surface Hopping as a Tool for Modeling Condensed Phase Linear Absorption Spectra. J. Chem. Theory Comput. 2015, 11, 4328-4341.

(412) Petit, A. S.; Subotnik, J. E. Calculating Time-Resolved Differential Absorbance Spectra for Ultrafast Pump-Probe Experiments with Surface Hopping Trajectories. J. Chem. Phys. 2014, $141,154108$.

(413) Santoro, F.; Improta, R.; Lami, A.; Bloino, J.; Barone, V. Effective Method to Compute Franck-Condon Integrals for Optical Spectra of Large Molecules in Solution. J. Chem. Phys. 2007, 126, 084509.

(414) Rohr, M. I. S.; Mitric, R.; Petersen, J. Vibrationally Resolved Optical Spectra and Ultrafast Electronic Relaxation Dynamics of Diamantane. Phys. Chem. Chem. Phys. 2016, 18, 8701-8709. (415) Polli, D.; Rivalta, I.; Nenov, A.; Weingart, O.; Garavelli, M.; Cerullo, G. Tracking the Primary Photoconversion Events in Rhodopsins by Ultrafast Optical Spectroscopy. Photochem. Photobiol. Sci. 2015, 14, 213-228.

(416) Gozem, S.; Gunina, A. O.; Ichino, T.; Osborn, D. L.; Stanton, J. F.; Krylov, A. I. Photoelectron Wave Function in Photoionization: Plane Wave or Coulomb Wave? J. Phys. Chem. Lett. 2015, 6, 4532-4540.

(417) Oana, C. M.; Krylov, A. I. Dyson Orbitals for Ionization from the Ground and Electronically Excited States within Equation-of-Motion Coupled-Cluster Formalism: Theory, Implementation, and Examples. J. Chem. Phys. 2007, 127, 234106.

(418) Ponzi, A.; Sapunar, M.; Angeli, C.; Cimiraglia, R.; Došlić, N.; Decleva, P. Photoionization of Furan from the Ground and Excited Electronic States. J. Chem. Phys. 2016, 144, 084307.

(419) Stolow, A. Femtosecond Time-Resolved Photoelectron Spectroscopy of Polyatomic Molecules. Annu. Rev. Phys. Chem. 2003, 54, 89-119.

(420) Zewail, A. H. Femtochemistry: Atomic-Scale Dynamics of the Chemical Bond. J. Phys. Chem. A 2000, 104, 5660-5694.

(421) Fingerhut, B. P.; Dorfman, K. E.; Mukamel, S. Monitoring Nonadiabatic Dynamics of the RNA Base Uracil by UV Pump-IR Probe Spectroscopy. J. Phys. Chem. Lett. 2013, 4, 1933-1942. (422) Petrone, A.; Williams-Young, D. B.; Lingerfelt, D. B.; Li, X. Ab Initio Excited-State Transient Raman Analysis. J. Phys. Chem. A 2017, 121, 3958-3965. 
(423) Liu, J.; Liang, W. Analytical Approach for the Excited-State Hessian in Time-Dependent Density Functional Theory: Formalism, Implementation, and Performance. J. Chem. Phys. 2011, $135,184111$.

(424) Mukamel, S. Multidimensional Femtosecond Correlation Spectroscopies of Electronic and Vibrational Excitations. Annu. Rev. Phys. Chem. 2000, 51, 691-729.

(425) Rivalta, I.; Nenov, A.; Weingart, O.; Cerullo, G.; Garavelli, M.; Mukamel, S. Modelling Time-Resolved Two-Dimensional Electronic Spectroscopy of the Primary Photoisomerization Event in Rhodopsin. J. Phys. Chem. B 2014, 118, 8396-8405.

(426) Nenov, A.; Giussani, A.; Segarra-Martí, J.; Jaiswal, V. K.; Rivalta, I.; Cerullo, G.; Mukamel, S.; Garavelli, M. Modeling the High-Energy Electronic State Manifold of Adenine: Calibration for Nonlinear Electronic Spectroscopy. J. Chem. Phys. 2015, 142, 212443.

(427) Tempelaar, R.; van der Vegte, C. P.; Knoester, J.; Jansen, T. L. C. Surface Hopping Modeling of Two-Dimensional Spectra. J. Chem. Phys. 2013, 138, 164106.

(428) Altoè, P.; Stenta, M.; Bottoni, A.; Garavelli, M. A Tunable QM/MM Approach to Chemical Reactivity, Structure and Physico-Chemical Properties Prediction. Theor. Chem. Acc. 2007, 118, 219-240.

(429) Toniolo, A.; Granucci, G.; Martínez, T. J. Conical Intersections in Solution: A QM/MM Study Using Floating Occupation Semiempirical Configuration Interaction Wave Functions. J. Phys. Chem. A 2003, 107, 3822-3830.

(430) Humeniuk, A.; Mitrić, R. DFTBaby: A Software Package for Non-adiabatic Molecular Dynamics Simulations Based on Long-Range Corrected Tight-binding TD-DFT(B). Comput. Phys. Commun. 2017, 221, 174-202.

(431) Du, L.; Lan, Z. An On-the-Fly Surface-Hopping Program JADE for Nonadiabatic Molecular Dynamics of Polyatomic Systems: Implementation and Applications. J. Chem. Theory Comput. 2015, 11, 1360-1374.

(432) Akimov, A. V. Libra: An Open-Source "Methodology Discovery" Library for Quantum and Classical Dynamics Simulations. J. Comput. Chem. 2016, 37, 1626-1649.

(433) Barbatti, M.; Granucci, G.; Persico, M.; Ruckenbauer, M.; Vazdar, M.; Eckert-Maksić, M.; Lischka, H. The on-the-Fly Surface-Hopping Program System Newton-X: Application to Ab Initio Simulation of the Nonadiabatic Photodynamics of Benchmark Systems. J. Photochem. Photobiol., A 2007, 190, 228-240.

(434) Barbatti, M.; Ruckenbauer, M.; Plasser, F.; Pittner, J.; Granucci, G.; Persico, M.; Lischka, H. Newton-X: A Surface-Hopping Program for Nonadiabatic Molecular Dynamics. WIREs: Comp. Mol. Sci. 2014, 4, 26-33.

(435) Tavernelli, I. Electronic Density Response of Liquid Water Using Time-Dependent Density Functional Theory. Phys. Rev. B 2006, 73, 094204.

(436) Gaenko, A.; DeFusco, A.; Varganov, S. A.; Martínez, T. J.; Gordon, M. S. Interfacing the Ab Initio Multiple Spawning Method with Electronic Structure Methods in GAMESS: Photodecay of trans-Azomethane. J. Phys. Chem. A 2014, 118, 10902-10908.

(437) Schmidt, M. W.; Baldridge, K. K.; Boatz, J. A.; Elbert, S. T.; Gordon, M. S.; Jensen, J. H.; Koseki, S.; Matsunaga, N.; Nguyen, K. A.; Su, S., et al. General Atomic and Molecular Electronic Structure System. J. Comput. Chem. 1993, 14, 1347-1363.

(438) Mortensen, J. J.; Hansen, L. B.; Jacobsen, K. W. Real-Space Grid Implementation of the Projector Augmented Wave Method. Phys. Rev. B 2005, 71, 035109.

(439) Sherwood, P.; de Vries, A. H.; Guest, M. F.; Schreckenbach, G.; Catlow, C. R. A.; French, S. A.; Sokol, A. A.; Bromley, S. T.; Thiel, W.; Turner, A. J., et al. QUASI: A General Purpose 
Implementation of the QM/MM Approach and Its Application to Problems in Catalysis. J. Mol. Struct. THEOCHEM 2003, 632, 1-28.

(440) Aquilante, F.; Autschbach, J.; Carlson, R. K.; Chibotaru, L. F.; Delcey, M. G.; De Vico, L.; Fdez. Galván, I.; Ferré, N.; Frutos, L. M.; Gagliardi, L., et al. Molcas 8: New Capabilities for Multiconfigurational Quantum Chemical Calculations Across the Periodic Table. J. Comput. Chem. 2016, 37, 506-541.

(441) Werner, H.-J.; Knowles, P. J.; Knizia, G.; Manby, F. R.; Schütz, M. Molpro: A GeneralPurpose Quantum Chemistry Program Package. Wiley Interdisciplinary Reviews: Computational Molecular Science 2012, 2, 242-253.

(442) Toniolo, A.; Olsen, S.; Manohar, L.; Martínez, T. J. Conical Intersection Dynamics in Solution: The Chromophore of Green Fluorescent Protein. Faraday Discuss. 2004, 127, 149-163. (443) Xavier, A.; Joseba, A.-R.; David, A. S.; Micael, J. T. O.; Fernando, N.; Alberto, C.; Javier, M.; Agustin, A.; Steven, G. L.; Alán, A.-G., et al. Time-Dependent Density-Functional Theory in Massively Parallel Computer Architectures: The Octopus Project. J. Phys.: Condens. Matter 2012, 24, 233202.

(444) Tapavicza, E.; Meyer, A. M.; Furche, F. Unravelling the Details of Vitamin D Photosynthesis by Non-Adiabatic Molecular Dynamics Simulations. Phys. Chem. Chem. Phys. 2011, 13, 20986-20998.

(445) Ahlrichs, R.; Bär, M.; Häser, M.; Horn, H.; Kölmel, C. Electronic-Structure Calculations on Workstation Computers - the Program System Turbomole. Chem. Phys. Lett. 1989, 162, 165169.

(446) Shao, Y.; Gan, Z.; Epifanovsky, E.; Gilbert, A. T. B.; Wormit, M.; Kussmann, J.; Lange, A. W.; Behn, A.; Deng, J.; Feng, X., et al. Advances in Molecular Quantum Chemistry Contained in the Q-Chem 4 Program Package. Mol. Phys. 2015, 113, 184-215.

(447) Lan, Z.; Lu, Y.; Fabiano, E.; Thiel, W. QM/MM Nonadiabatic Decay Dynamics of 9HAdenine in Aqueous Solution. ChemPhysChem 2011, 12, 1989-1998.

(448) Ruckenbauer, M.; Barbatti, M.; Muller, T.; Lischka, H. Nonadiabatic Excited-State Dynamics with Hybrid ab Initio Quantum-Mechanical/Molecular-Mechanical Methods: Solvation of the Pentadieniminium Cation in Apolar Media. J. Phys. Chem. A 2010, 114, 6757-6765.

(449) Conti, I.; Altoe, P.; Stenta, M.; Garavelli, M.; Orlandi, G. Adenine Deactivation in DNA Resolved at the CASPT2//CASSCF/AMBER Level. Phys. Chem. Chem. Phys. 2010, 12, 50165023.

(450) Ferretti, A.; Granucci, G.; Lami, A.; Persico, M.; Villani, G. Quantum mechanical and semiclassical dynamics at a conical intersection. J. Chem. Phys. 1996, 104, 5517-5527.

(451) Leggett, A. J.; Chakravarty, S.; Dorsey, A. T.; Fisher, M. P. A.; Garg, A.; Zwerger, W. Dynamics of the dissipative two-state system. Reviews of Modern Physics 1987, 59, 1-85.

(452) Metz, S.; Kästner, J.; Sokol, A. A.; Keal, T. W.; Sherwood, P. Chemshell—a Modular Software Package for QM/MM Simulations. WIREs: Comp. Mol. Sci. 2014, 4, 101-110.

(453) Zheng, J.; Li, Z.-H.; Jasper, A. W.; Bonhommeau, D. A.; Valero, R.; Meana-Pañeda, R.; Mielke, S. L.; Truhlar, D. G. ANT: A Program for Adiabatic and Nonadiabatic Trajectories. 2016, http://comp.chem.umn.edu/ant.

(454) Weingart, O.; Lan, Z.; Koslowski, A.; Thiel, W. Chiral Pathways and Periodic Decay in cis-Azobenzene Photodynamics. J. Phys. Chem. Lett. 2011, 2, 1506-1509.

(455) Li, X.; Xie, Y.; Hu, D.; Lan, Z. Analysis of the Geometrical Evolution in On-the-Fly Surface-Hopping Nonadiabatic Dynamics with Machine Learning Dimensionality Reduction 
Approaches: Classical Multidimensional Scaling and Isometric Mapping. J. Chem. Theory Comput. 2017.

(456) Virshup, A. M.; Chen, J.; Martínez, T. J. Nonlinear Dimensionality Reduction for Nonadiabatic Dynamics: The Influence of Conical Intersection Topography on Population Transfer Rates. J. Chem. Phys. 2012, 137, 22A519.

(457) Barbatti, M.; Ruckenbauer, M.; Lischka, H. The Photodynamics of Ethylene: A SurfaceHopping Study on Structural Aspects. J. Chem. Phys. 2005, 122, 174307.

(458) Barbatti, M.; Lan, Z.; Crespo-Otero, R.; Szymczak, J. J.; Lischka, H.; Thiel, W. Critical Appraisal of Excited State Nonadiabatic Dynamics Simulations of 9H-Adenine. J. Chem. Phys. 2012, 137, 22A503-14.

(459) Grabarek, D.; Walczak, E.; Andruniów, T. Assessing the Accuracy of Various Ab Initio Methods for Geometries and Excitation Energies of Retinal Chromophore Minimal Model by Comparison with CASPT3 Results. J. Chem. Theory Comput. 2016, 12, 2346-2356.

(460) Send, R.; Sundholm, D. Stairway to the Conical Intersection: A Computational Study of the Retinal Isomerization. J. Phys. Chem. A 2007, 111, 8766-8773.

(461) Evans, N. L.; Ullrich, S. Wavelength Dependence of Electronic Relaxation in Isolated Adenine Using UV Femtosecond Time-Resolved Photoelectron Spectroscopy. J. Phys. Chem. A 2010, 114, 11225-11230.

(462) Chen, H.-T.; Reichman, D. R. On the Accuracy of Surface Hopping Dynamics in Condensed Phase Non-Adiabatic Problems. J. Chem. Phys. 2016, 144, 094104.

(463) Ben-Nun, M.; Martínez, T. J. A Continuous Spawning Method for Nonadiabatic Dynamics and Validation for the Zero-Temperature Spin-Boson Problem. Isr. J. Chem. 2007, 47, 75-88.

(464) Szymczak, J. J.; Barbatti, M.; Soo Hoo, J. T.; Adkins, J. A.; Windus, T. L.; Nachtigallová, D.; Lischka, H. Photodynamics Simulations of Thymine: Relaxation into the First Excited Singlet State. J. Phys. Chem. A 2009, 113, 12686-12693.

(465) Hack, M. D.; Wensmann, A. M.; Truhlar, D. G.; Ben-Nun, M.; Martínez, T. J. Comparison of Full Multiple Spawning, Trajectory Surface Hopping, and Converged Quantum Mechanics for Electronically Nonadiabatic Dynamics. J. Chem. Phys. 2001, 115, 1172-1186.

(466) Tully, J. C. Perspective: Nonadiabatic Dynamics Theory. J. Chem. Phys. 2012, 137, 22A301.

(467) Barbatti, M.; Sen, K. Effects of Different Initial Condition Samplings on Photodynamics and Spectrum of Pyrrole. Int. J. Quantum Chem. 2016, 116, 762-771.

(468) Miller, W. H.; Hase, W. L.; Darling, C. L. A Simple Model for Correcting the Zero Point Energy Problem in Classical Trajectory Simulations of Polyatomic Molecules. J. Chem. Phys. 1989, 91, 2863-2868.

(469) Xie, Z.; Bowman, J. M. Zero-Point Energy Constraint in Quasi-classical Trajectory Calculations. J. Phys. Chem. A 2006, 110, 5446-5449.

(470) Sobolewski, A. L.; Domcke, W.; Dedonder-Lardeux, C.; Jouvet, C. Excited-State Hydrogen Detachment and Hydrogen Transfer Driven by Repulsive ${ }^{1} \pi \sigma^{*}$ States: A New Paradigm for Nonradiative Decay in Aromatic Biomolecules. Phys. Chem. Chem. Phys. 2002, 4, 1093-1100. (471) Forsberg, N.; Malmqvist, P.-Å. Multiconfiguration Perturbation Theory with Imaginary Level Shift. Chem. Phys. Lett. 1997, 274, 196-204.

(472) Huix-Rotllant, M.; Nikiforov, A.; Thiel, W.; Filatov, M., Description of Conical Intersections with Density Functional Methods. In Density-Functional Methods for Excited States, Ferré, N.; Filatov, M.; Huix-Rotllant, M., Eds. Springer International Publishing: Cham, 2016; pp 445-476. 
(473) Fazzi, D.; Barbatti, M.; Thiel, W. Modeling Ultrafast Exciton Deactivation in Oligothiophenes via Nonadiabatic Dynamics. Phys. Chem. Chem. Phys. 2015, 17, 7787-7799.

(474) Douhal, A.; Kim, S. K.; Zewail, A. H. Femtosecond Molecular Dynamics of Tautomerization in Model Base Pairs. Nature 1995, 378, 260-263.

(475) Kwon, O.-H.; Zewail, A. H. Reply to Catalán: Double-Proton-Transfer Dynamics of PhotoExcited 7-Azaindole Dimers. Proc. Natl. Acad. Sci. USA 2008, 105, E79.

(476) Catalán, J.; del Valle, J. C.; Kasha, M. Resolution of Concerted Versus Sequential Mechanisms in Photo-Induced Double-Proton Transfer Reaction in 7-Azaindole H-Bonded Dimer. Proc. Natl. Acad. Sci. USA 1999, 96, 8338-8343.

(477) Kwon, O.-H.; Zewail, A. H. Double Proton Transfer Dynamics of Model DNA Base Pairs in the Condensed Phase. Proc. Natl. Acad. Sci. USA 2007, 104, 8703-8708.

(478) Chachisvilis, M.; Fiebig, T.; Douhal, A.; Zewail, A. H. Femtosecond Dynamics of a Hydrogen-Bonded Model Base Pair in the Condensed Phase: Double Proton Transfer in 7Azaindole. J. Phys. Chem. A 1998, 102, 669-673.

(479) Serrano-Andrés, L.; Merchán, M. Theoretical CASPT2 Study of the Excited State Double Proton Transfer Reaction in the 7-Azaindole Dimer. Chem. Phys. Lett. 2006, 418, 569-575.

(480) Yarkony, D. R. Nonadiabatic Quantum Chemistry—Past, Present, and Future. Chem. Rev. 2011, 112, 481-498.

(481) Matsika, S.; Krause, P. Nonadiabatic Events and Conical Intersections. Annu. Rev. Phys. Chem. 2011, 62, 621-643.

(482) Blumberger, J. Recent Advances in the Theory and Molecular Simulation of Biological Electron Transfer Reactions. Chem. Rev. 2015, 115, 11191-11238.

(483) Oberhofer, H.; Reuter, K.; Blumberger, J. Charge Transport in Molecular Materials: An Assessment of Computational Methods. Chem. Rev. 2017, 117, 10319-10357.

(484) Akimov, A. V.; Prezhdo, O. V. Large-Scale Computations in Chemistry: A Bird's Eye View of a Vibrant Field. Chem. Rev. 2015, 115, 5797-5890.

(485) Akimov, A. V.; Neukirch, A. J.; Prezhdo, O. V. Theoretical Insights into Photoinduced Charge Transfer and Catalysis at Oxide Interfaces. Chem. Rev. 2013, 113, 4496-4565.

(486) Kilina, S.; Kilin, D.; Tretiak, S. Light-Driven and Phonon-Assisted Dynamics in Organic and Semiconductor Nanostructures. Chem. Rev. 2015, 115, 5929-5978. 


\section{Biographies}

Rachel Crespo-Otero was born in Havana, Cuba. She earned her $\mathrm{PhD}$ at the University of Havana and the Autonomous University of Madrid supervised by Prof. Luis Alberto Montero (UH) and Prof. José Manuel García de la Vega (UAM). In 2010, she joined the group of Prof. Mario Barbatti at the Max Planck Institut für Kohlenforschung (Mülheim an der Ruhr, Germany), where she worked on the application and development of methods to investigate nonadiabatic processes. In 2013, she moved to the University of Bath (UK) for postdoctoral research on metastable materials and water splitting with Prof. Aron Walsh. Since January 2015, she is a Lecturer at Queen Mary University of London. Her research interests include the investigation of excited states and nonadiabatic phenomena at the frontier of Molecular and Materials Sciences. She contributes to the Newton-X program package.

Mario Barbatti was born and raised in Brazil. In 2001, he earned a Doctor of Sciences title in physics at the Federal University of Rio de Janeiro. In 2004, he moved to Vienna, where he was a postdoctoral fellow in the group of Hans Lischka and, in 2008, he earned a Habilitation to teach theoretical chemistry. In 2010, he became an independent group leader at the Max Planck Institut für Kohlenforschung in Mülheim an der Ruhr, Germany. Since 2015, he is a professor of the chair of excellence A*MIDEX at the University of Aix-Marseille in Marseille, France. For over fifteen years, Mario Barbatti has been working on the computational theoretical chemistry of molecular excited states. He is the designer and one of the leading developers of the Newton-X program package for NA-MQC dynamics, whose first release commemorated ten years in 2017. To this date, he has published over 120 articles. Among his principal scientific contributions are the first complete mapping of the deactivation pathways of UV-excited nucleobases using ab initio dynamics, the discovery of a new internal-conversion mechanism through electron transfer between solvent and chromophore, and the determination of the photochemical mechanism of a key prebiotic reaction. 


\section{Table of Contents Graph}

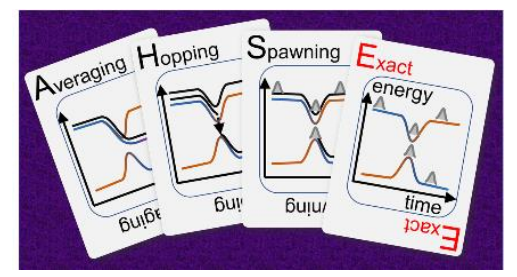

